\title{
DAMPING OF MAGNETOHYDRODYNAMIC TURBULENCE IN PARTIALLY IONIZED PLASMA: IMPLICATIONS FOR COSMIC RAY PROPAGATION
}

\author{
Siyao Xu ${ }^{1}$, Huirong Yan ${ }^{2,3,4}$, and A. Lazarian ${ }^{5}$ \\ ${ }^{1}$ Department of Astronomy, School of Physics, Peking University, Beijing 100871, China; syxu@pku.edu.cn \\ ${ }^{2}$ Kavli Institute for Astronomy and Astrophysics, Peking University, Beijing 100871, China \\ ${ }^{3}$ DESY, Platanenallee 6, D-15738 Zeuthen, Germany; huirong.yan@desy.de \\ ${ }^{4}$ Institut fur Physik und Astronomie, Universität Potsdam, D-14476 Potsdam, Germany \\ ${ }^{5}$ Department of Astronomy, University of Wisconsin, 475 North Charter Street, Madison, WI 53706, USA; lazarian@astro.wisc.edu \\ Received 2015 June 17; revised 2016 April 21; accepted 2016 April 22; published 2016 July 28
}

\begin{abstract}
We study the damping processes of both incompressible and compressible magnetohydrodynamic (MHD) turbulence in a partially ionized medium. We start from the linear analysis of MHD waves, applying both singlefluid and two-fluid treatments. The damping rates derived from the linear analysis are then used in determining the damping scales of MHD turbulence. The physical connection between the damping scale of MHD turbulence and the cutoff boundary of linear MHD waves is investigated. We find two branches of slow modes propagating in ions and neutrals, respectively, below the damping scale of slow MHD turbulence, and offer a thorough discussion of their propagation and dissipation behavior. Our analytical results are shown to be applicable in a variety of partially ionized interstellar medium (ISM) phases and the solar chromosphere. The importance of neutral viscosity in damping the Alfvenic turbulence in the interstellar warm neutral medium and the solar chromosphere is demonstrated. As a significant astrophysical utility, we introduce damping effects to the propagation of cosmic rays in partially ionized ISM. The important role of turbulence damping in both transit-time damping and gyroresonance is identified.
\end{abstract}

Key words: cosmic rays - magnetohydrodynamics (MHD) - turbulence

\section{INTRODUCTION}

Partially ionized plasmas are universal in both space and astrophysical environments. They fill a substantial volume throughout the Galaxy and build up molecular clouds (MCs) from which stars form. The presence of neutrals affects the plasma dynamics indirectly, mediated by the friction between ions and neutrals (see the studies by, e.g., Piddington 1956; Kulsrud \& Pearce 1969).

Astrophysical plasmas are generally turbulent and magnetized (see, e.g., Armstrong et al. 1995; Chepurnov \& Lazarian 2010). Both incompressible and compressible magnetohydrodynamic (MHD) turbulences have been studied previously (Goldreich \& Sridhar 1995, hereafter GS95; Lazarian \& Vishniac 1999; Lithwick \& Goldreich 2001). The GS95 model of turbulence and its extensions in compressible fluids successfully reproduce the properties of MHD turbulence and are supported by numerical tests (Maron \& Goldreich 2001; Cho et al. 2002; Cho \& Lazarian 2002, 2003; Kowal \& Lazarian 2010; Beresnyak 2014, 2015). A realistic treatment of MHD turbulence is crucial for understanding many fundamental astrophysical problems (Yan \& Lazarian 2004; Lazarian 2006; Lazarian et al. 2012; Lazarian \& Yan 2014).

The turbulence theory for a partially ionized medium should be constructed under consideration of the damping by neutrals. There are extensive studies in the literature regarding ionneutral collisional damping, using either single-fluid (e.g., Braginskii 1965; Balsara 1996; Khodachenko et al. 2004; Forteza et al. 2007) or two-fluid descriptions (e.g., Pudritz 1990; Balsara 1996; Kumar \& Roberts 2003; Mouschovias et al. 2011; Zaqarashvili et al. 2011; Soler et al. 2013a, 2013b). The single-fluid approach has a simpler formalism and hence physical transparency, but is restricted to the limited wave frequencies lower than the neutral-ion collisional frequency.
The two-fluid approach treats ion-electron and neutral fluids separately and is valid over a broad range of wave frequencies.

But all these studies are restricted to linear MHD waves. The modern advances in the study of MHD turbulence reveal that it is a highly nonlinear phenomenon, with the energy injected at a large scale cascading successively downwards at the turbulence eddy turnover rate (see, e.g., Cho \& Lazarian 2003; Cho et al. 2003, pp. 56-98). The effect of ion-neutral collisions on the cascade of compressible MHD turbulence was investigated by Lithwick \& Goldreich (2001), but they mainly focused on a highly ionized medium with gas pressure overwhelming magnetic pressure. The role of neutral-viscous damping of turbulent motions in partially ionized gas was thoroughly studied in Lazarian et al. (2004). Combining the two damping effects, a recent work by Xu et al. (2014) (hereafter Paper I) carried out a detailed study of damping of the Alfvén component of MHD turbulence in a variety of turbulence regimes and demonstrated its significance in explaining the observed linewidth differences between neutrals and ions, and its potential value in measuring the magnetic field in MCs. In the current work, we extend the theory developed in Paper I to include compressible MHD turbulence. The weak coupling of Alfvén to compressible modes was suggested by GS95. The decomposition of MHD turbulence into Alfvén, fast, and slow modes has been confirmed and realized through a statistical procedure in both Fourier and real spaces (Cho \& Lazarian 2002, 2003; Kowal \& Lazarian 2010). On this basis, we can deal with the three components of MHD turbulence separately. The linear theory on wave propagation and dissipation in a partially ionized and magnetized medium has existed for long (Langer 1978). The concept of MHD turbulence offers new physical insights into the problem. Motivated and justified by the development of MHD turbulence theory, we investigate the damping processes of MHD turbulence in a partially ionized plasma. 
The output of this investigation has a wide range of astrophysical applications, for example for cosmic ray (CR) propagation. The propagation of CRs in the interstellar medium (ISM) is usually described in terms of diffusive transport of CRs in a turbulent magnetic field (see, e.g., Schlickeiser 2002). The well established statistics on the scattering of CRs by magnetic irregularities can successfully explain the high-degree isotropy of cosmic radiation. But the conventionally used, simple model of $\mathrm{CR}$ diffusion assuming a universal diffusion coefficient faces significant challenges in interpreting more and more observational findings (e.g., Hunter et al. 1997; Guillian et al. 2007; Adriani et al. 2011; Ackermann et al. 2012). With the development and acceptance of the modern concept of MHD turbulence (see the recent reviews by Brandenburg \& Lazarian 2014; Beresnyak \& Lazarian 2015), significant refinements and modifications have been progressively made in the picture of $\mathrm{CR}$ propagation, especially with respect to quantitatively relating the $\mathrm{CR}$ diffusion and the properties of the turbulent medium through which they pass.

The consideration of turbulence damping is necessary for a proper description of the interaction between CR particles and MHD turbulence. The studies of Yan \& Lazarian (2002, 2004, 2008) showed the important influence of the properties of turbulence and its damping on CR scattering and propagation in a fully ionized plasma. The ionization fraction possessed by the ISM varies over a vast range, from fully ionized to almost entirely neutral media (Spitzer 1978; Draine 2011). In the presence of neutrals, the damping processes in a partially ionized medium is completely different from that in a fully ionized medium. Accordingly, the propagation of CRs in a partially ionized medium is likely to present distinctive features and deserves special attention. With this motivation, we extend the earlier research in the partially ionized medium and aim to achieve a realistic model of CR transport in the ISM.

The organization of this paper is as follows. In Section 2, we introduce the concept of neutral-ion and ion-neutral decoupling. We then provide a linear analysis of MHD waves using both two-fluid and single-fluid approaches in Section 3. With the damping rates obtained from linear analysis, damping of MHD turbulence is presented in Section 4. The numerical tests of our analytical results under diverse partially ionized ISM conditions and in the solar chromosphere (SC) are given in Section 5. Section 6 contains an application to $\mathrm{CR}$ propagation in a partially ionized medium. Section 7 presents further discussion. The summary is given in Section 8.

\section{COUPLING BETWEEN NEUTRALS AND IONS}

The coupling of neutrals and ions depends on the wave frequency $\omega$ of interest in comparison with the neutral-ion collision frequency,

$$
\nu_{n i}=\gamma_{d} \rho_{i}
$$

Here $\rho_{i}$ is the mass density of ions and $\gamma_{d}$ is the drag coefficient defined in Shu (1992). $\nu_{n i}$ is related to ion-neutral collision frequency $\nu_{i n}$ by

$$
\nu_{n i} \rho_{n}=\nu_{\text {in }} \rho_{i},
$$

where $\rho_{n}$ is the mass density of neutrals. The conditions $\omega \sim \nu_{n i}$ and $\omega \sim \nu_{\text {in }}$ correspond to the neutral-ion decoupling scale $k_{\text {dec, } n i}^{-1}$ and the ion-neutral decoupling scale $k_{\mathrm{dec}, \text { in }}^{-1}$, respectively. In a predominantly neutral medium, $k_{\mathrm{dec}, n i}^{-1}$ is much larger than $k_{\mathrm{dec}, i n}^{-1}$. At greater scales than $k_{\mathrm{dec}, n i}^{-1}$, neutrals and ions are perfectly
Table 1

Decoupling Scales of MHD Waves

\begin{tabular}{lcc}
\hline \hline & $k_{\mathrm{dec}, n i}$ & $k_{\mathrm{dec}, i n}$ \\
\hline Alfvén & $\frac{\nu_{n i}}{V_{A} \cos \theta}$ & $\frac{\nu_{i n}}{V_{A i} \cos \theta}$ \\
Fast & $\frac{\nu_{n i}}{V_{A}}$ & $\frac{\nu_{i n}}{V_{A i}}$ \\
Slow & $\frac{\nu_{n i}}{c_{s n}}$ & $\frac{\nu_{i n}}{c_{s i} \cos \theta}$
\end{tabular}

coupled and oscillate together. In this regime the single-fluid approximation is adequate to study the behavior and damping of MHD waves. Below $k_{\mathrm{dec}, n i}^{-1}$, neutrals start to decouple from ions. Perturbations of the magnetic field cannot be instantly transmitted to neutrals, thus Alfvén waves are suppressed in the neutral fluid.

The expressions of $k_{\mathrm{dec}}$ are different for different wave modes, depending on the form of their respective wave frequencies. In the limit case of low- $\beta$, where $\beta=2 c_{s}^{2} / V_{A}^{2}$ is defined as the ratio of gas and magnetic pressure, both Alfvén and fast modes propagate with Alfvén speed. Their neutral-ion decoupling scales are determined by the conditions $V_{A} k \cos \theta=\nu_{n i}$ and $V_{A} k=\nu_{n i}$, respectively, where $\theta$ is the wave propagation angle relative to the magnetic field. $\cos \theta$ appears for Alfvén modes because their waves travel along the magnetic field, while fast waves propagate isotropically and their decoupling scales do not depend on the propagation direction. The intrinsic nature of neutrals allows them to support their own sound waves after they decouple from ions. Once they satisfy the condition $c_{s n} k=\nu_{n i}$, neutrals are able to develop their sound waves and become independent from ion modulation. Thus $k_{\mathrm{dec}, n i}^{-1}$ of slow modes depends on $\omega$ of sound waves. So we can summarize that in low$\beta$ plasmas, the neutral-ion decoupling scale sets the largest scale for the absence of Alfvén waves in neutral fluids for Alfvén and fast modes, and the presence of sound waves in neutrals in the case of slow modes.

In the meantime, ions are still subject to frequent collisions with surrounding neutrals above the scale $k_{\mathrm{dec}, \text { in }}^{-1}$. Therefore the remaining wave motions in ions can be strongly influenced by the collisional friction. On the other hand, the single-fluid approximation can still be used to describe the MHD waves since ions maintain the coupling state with neutrals. At shorter scales than $k_{\mathrm{dec}, \text { in }}^{-1}$ ions also become ineffectively coupled with neutrals. The scale $k_{\mathrm{dec}, \text { in }}^{-1}$ is determined by $\omega$ of MHD waves in ions alone. For the weakly coupled ions and neutrals, the assumption of strong coupling breaks down, so the two-fluid description with no restriction imposed on the wave frequency range needs to be adopted. In the following, we use "strongly" and "weakly" coupled regimes to mean $k<k_{\mathrm{dec}, n i}$ and $k>k_{\mathrm{dec}, i n}$, respectively.

Based on the above analysis, Table 1 lists the decoupling scales for different wave modes and regimes.

\section{MHD WAVES IN A PARTIALLY IONIZED PLASMA}

The study of damping processes is based on the linear analysis of MHD waves in a partially ionized plasma. We present the analytical expressions for the damping rate obtained from both two-fluid and single-fluid approaches, and mainly focus on the solutions in a low- $\beta$ limit.

\subsection{The Two-fluid Approach}

Alfvén waves. In a neutral-dominated medium, the dissipation of Alfvén waves can be enhanced by both ion-neutral collisional damping and the viscosity effects in neutrals. The 
kinematic viscosity of neutrals is usually used to determine the viscous dissipation cutoff of hydrodynamic turbulence in partially ionized gas, as the collisional cross-section of neutrals is much smaller than the Coulomb cross-section of ions (Kulsrud \& Anderson 1992). Lazarian et al. (2004) took into account that most of the velocity gradients in Alfvénic turbulence are perpendicular to the local direction of the magnetic field and in this approximation showed the importance of neutral viscosity for the damping effect of MHD turbulence. A comparative study of ion-neutral collisional and neutral-viscous damping was then performed in Paper I.

The relative importance of the two damping mechanisms can vary in different phases of the ISM. When the ion-neutral collisional damping is much more efficient and viscous damping can be safely neglected, Alfvén waves have a wellstudied dispersion relation (see, e.g., Piddington 1956; Kulsrud $\&$ Pearce 1969; Soler et al. 2013b),

$$
\omega^{3}+i(1+\chi) \nu_{n i} \omega^{2}-k^{2} \cos ^{2} \theta V_{A i}^{2} \omega-i \nu_{n i} k^{2} \cos ^{2} \theta V_{A i}^{2}=0,
$$

where $\chi=\rho_{n} / \rho_{i}$. The solution is complex, $\omega=\omega_{R}+i \omega_{I}$. Under the assumption of weak damping $\left|\omega_{I}\right| \ll\left|\omega_{R}\right|$, we approximately obtain

$$
\begin{gathered}
\omega_{R}^{2}=\frac{k^{2} \cos ^{2} \theta V_{A i}^{2}\left((1+\chi) \nu_{n i}^{2}+k^{2} \cos ^{2} \theta V_{A i}^{2}\right)}{(1+\chi)^{2} \nu_{n i}^{2}+k^{2} \cos ^{2} \theta V_{A i}^{2}}, \\
\omega_{I}=-\frac{\nu_{n i} \chi k^{2} \cos ^{2} \theta V_{A i}^{2}}{2\left((1+\chi)^{2} \nu_{n i}^{2}+k^{2} \cos ^{2} \theta V_{A i}^{2}\right)} .
\end{gathered}
$$

It is reduced to

$$
\begin{gathered}
\omega_{R}^{2}=V_{A}^{2} k^{2} \cos ^{2} \theta, \\
\omega_{I}=-\frac{\xi_{n} V_{A}^{2} k^{2} \cos ^{2} \theta}{2 \nu_{n i}}
\end{gathered}
$$

in the strongly coupled regime, i.e., $\omega \ll \nu_{n i}$, and

$$
\begin{gathered}
\omega_{R}^{2}=V_{A i}^{2} k^{2} \cos ^{2} \theta, \\
\omega_{I}=-\frac{\nu_{i n}}{2}
\end{gathered}
$$

in the weakly coupled regime, i.e., $\omega \gg \nu_{\text {in }}$. Here we define the neutral fraction as $\xi_{n}=\rho_{n} / \rho=\chi /(1+\chi)$.

The assumption of weak damping holds in both the strongly and weakly coupled regimes, but fails at intermediate wave frequencies. The strongly damped region can be confined by the condition $\left|\omega_{I}\right|=\left|\omega_{R}\right|$. Equations (5) and (6) yield the boundary wavenumbers,

$$
\begin{aligned}
k_{c}^{+} & =\frac{2 \nu_{n i}}{V_{A} \xi_{n} \cos \theta}, \\
k_{c}^{-} & =\frac{\nu_{i n}}{2 V_{A i} \cos \theta} .
\end{aligned}
$$

$\left|\omega_{I}\right|$ converges with $\left|\omega_{R}\right|$ at $k_{c}^{ \pm}$. Within $\left[k_{c}^{+}, k_{c}^{-}\right]$, the Alfvén waves become nonpropagating and have purely imaginary wave frequencies. Although the precise calculation of the cutoff interval of Alfvén waves should involve the discriminant of Equation (3) (e.g., Soler et al. 2013b), numerical results confirm that the boundaries $\left[k_{c}^{+}, k_{c}^{-}\right]$obtained from the equality between $\left|\omega_{I}\right|$ and $\left|\omega_{R}\right|$ can confine the nonpropagating interval with $\omega_{R}=0$ very well. Therefore we choose this simple derivation and use the resulting $k_{c}^{ \pm}$as the limit wavenumbers of the cutoff region.

By comparison with Table 1, we find that the cutoff boundaries are connected with the decoupling scales by

$$
\begin{aligned}
& k_{c, \|}^{+}=\frac{2}{\xi_{n}} k_{\mathrm{dec}, n i, \|,} \\
& k_{c, \|}^{-}=\frac{1}{2} k_{\mathrm{dec}, i n, \|,}
\end{aligned}
$$

showing that the cutoff region is slightly smaller than the $\left[k_{\mathrm{dec}, n i}, k_{\mathrm{dec}, i n}\right]$ range.

When neutral viscosity dominates over the ion-neutral collisional damping, the expression for the damping rate in the strongly coupled regime can approximately reduce to (see Equation (21b) in Paper I for the exact expression)

$$
\left|\omega_{I}\right|=\tau_{\nu}^{-1}=k^{2} \nu_{n},
$$

where $\nu_{n}=v_{\text {th }} /\left(n_{n} \sigma_{n n}\right)$ is the kinematic viscosity in neutrals, and $v_{\text {th }}=\sqrt{2 k_{B} T / m_{n}}$ is neutral thermal speed. Note that unlike ion viscosity, neutral viscosity is unaffected by the magnetic field and has an isotropic coefficient (Soler et al. 2015). ${ }^{6}$ By equaling the above $\left|\omega_{I}\right|$ and $\left|\omega_{R}\right|$ from Equation (5a), the corresponding cutoff wavenumber is

$$
k_{c}^{+}=\frac{V_{A} \cos \theta}{\nu_{n}} \text {. }
$$

The wave frequencies and $k_{c}^{-}$in the weakly coupled regime are the same as those in the case of ion-neutral collisional damping.

Fast and slow waves. We then turn to the magnetoacoustic waves. Differing from Alfvén waves, the magnetoacoustic waves are compressible and do not produce efficient shear motions that induce neutral viscosity. The comparison between the neutralviscous and ion-neutral collisional damping rates presented in Paper I confirms that neutral viscosity is unimportant in damping magnetoacoustic waves. Therefore, we consider ion-neutral collisional friction as the dominant damping mechanism and disregard the effect of neutral viscosity in the case of magnetoacoustic waves. We adopt the dispersion relation given by Equation (51) in Soler et al. (2013a) (see also Equation (57) in Zaqarashvili et al. 2011). Taking advantage of the weak damping assumption, analytic solutions can be obtained in the limit case of strongly coupled fluids,

$$
\begin{gathered}
\omega_{R}^{2}=\frac{1}{2}\left[\left(c_{s}^{2}+V_{A}^{2}\right) \pm \sqrt{\left(c_{s}^{2}+V_{A}^{2}\right)^{2}-4 c_{s}^{2} V_{A}^{2} \cos ^{2} \theta}\right] k^{2}, \\
\omega_{I}=-\frac{k^{2}\left[\xi_{n} V_{A}^{2}\left(c_{s}^{2} k^{2}-\omega_{R}^{2}\right)+\xi_{i} c_{s}^{2} \omega_{R}^{2}\right]}{2 \nu_{n i}\left[k^{2}\left(c_{s}^{2}+V_{A}^{2}\right)-2 \omega_{R}^{2}\right]} .
\end{gathered}
$$

Here $c_{s}=\sqrt{c_{s i}^{2} \xi_{i}+c_{s n}^{2} \xi_{n}}$ is the sound speed in strongly coupled ions and neutrals, and $\xi_{i}=1-\xi_{n}=1 /(1+\chi)$ is the ion fraction. The above solutions are consistent with those

\footnotetext{
6 The neutral viscosity used in this work is different from that introduced in Vranjes (2014). Under the condition that the dynamics of neutrals are regulated by the magnetic field and ion gyro-effects, they derived a neutral viscosity tensor coupled to that of ions. As expected, the resulting neutral-viscous damping in their work is always negligible compared with ion-neutral collisional damping.
} 
in an earlier work, e.g., Ferriere et al. (1988). Different from Alfvén waves, the compressibility introduces the important parameter $\beta$. Its definition was given in Section 2. In a low- $\beta$ plasma, the solutions in strongly coupled regime have simple expressions,

$$
\begin{gathered}
\omega_{R}^{2}=V_{A}^{2} k^{2}, \\
\omega_{I}=-\frac{\xi_{n} V_{A}^{2} k^{2}}{2 \nu_{n i}}
\end{gathered}
$$

for fast modes, and

$$
\begin{gathered}
\omega_{R}^{2}=c_{s}^{2} k^{2} \cos ^{2} \theta, \\
\omega_{I}=-\frac{\xi_{n} c_{s}^{2} k_{\perp}^{2}}{2 \nu_{n i}}
\end{gathered}
$$

for slow modes.

In the weakly coupled regime, under low- $\beta$ conditions, the propagating component of the wave frequency becomes

$$
\omega_{R}^{2}=V_{A i}^{2} k^{2}
$$

for fast modes, and

$$
\omega_{R}^{2}=c_{s i}^{2} k^{2} \cos ^{2} \theta
$$

for slow modes. They have the same damping rate as Alfvén waves at high wave frequencies (Equation (6b)).

If we compare the expressions of $\omega_{I}$ of the three modes in the strongly coupled regime, we find that the Alfvén and fast modes both have a damping rate proportional to their quadratic wave frequencies (Equations (5b), (12b)), as they can always induce oscillations of ions orthogonal to the magnetic field. The additional magnetic restoring force exerted on ions results in relative drift between ions and neutrals and damping to the wave motions. It indicates that faster propagating waves are more strongly damped. But for slow modes, we see from Equation $(13 b)$ that there is no damping to purely parallel propagation with $\theta=0$, since in a low- $\beta$ plasma, slow modes propagating along the magnetic field are analogous to sound waves in the absence of a magnetic field, and hence undamped.

We then compare the expressions of $\omega_{I}$ in strongly and weakly coupled regimes. We find the neutral-ion collisions at low wave frequencies are responsible for coupling the two fluids together, and more frequent collisions bring weaker damping to MHD waves. Differently, the damping rate in the weak coupling regime (Equation (6b)) purely depends on ionneutral collisional frequency. The collisional friction plays the role of dissipating waves in ions.

Analogically, the cutoff boundaries can be determined from $\left|\omega_{I}\right|=\left|\omega_{R}\right|$. Fast modes have

$$
\begin{aligned}
& k_{c}^{+}=\frac{2 \nu_{n i}}{V_{A} \xi_{n}}, \\
& k_{c}^{-}=\frac{\nu_{i n}}{2 V_{A i}},
\end{aligned}
$$

which are the same as Equation (7) for Alfvén waves except $k_{c, \|}^{ \pm}$are replaced by $k_{c}^{ \pm}$. Slow modes have

$$
\begin{aligned}
k_{c}^{+} & =\frac{2 \nu_{n i} \cos \theta}{c_{s} \xi_{n} \sin ^{2} \theta}, \\
k_{c}^{-} & =\frac{\nu_{i n}}{2 c_{s i} \cos \theta} .
\end{aligned}
$$

The relations between $k_{c}^{ \pm}$and the decoupling scales of fast waves (see Table 1) are similar to those of Alfvén waves in the direction parallel to the magnetic field,

$$
\begin{aligned}
& k_{c}^{+}=\frac{2}{\xi_{n}} k_{\mathrm{dec}, n i}, \\
& k_{c}^{-}=\frac{1}{2} k_{\mathrm{dec}, i n} .
\end{aligned}
$$

Slow waves have the same $k_{c, \|}^{-}=\frac{1}{2} k_{\mathrm{dec}, i n, \|}$ as Alfvén waves, but the relation between $k_{c}^{+}$and $k_{\mathrm{dec}, n i}$ is less straightforward. We will discuss this character of slow modes in Section 4.

In fact, in the context of anisotropic MHD turbulence, the wave propagation angle $\theta$ appearing in $k_{c}^{ \pm}$and the decoupling scales also depend on the scales. In Section 4 we will present their exact expressions.

\subsection{The Single-fluid Approach}

Although the single-fluid approach has a restriction on the range of wave frequencies, i.e., $\omega<\nu_{i n}$, it has the advantage of a simpler mathematical treatment. We take the dispersion relations from Balsara (1996) under the single-fluid approximation. We also follow the approximation of negligible ion density applied in their work for this subsection.

The dispersion relation of Alfvén waves is

$$
\lambda^{2}+i \tilde{k} V_{A n} \cos \theta \lambda-V_{A n}^{2} \cos ^{2} \theta=0 .
$$

The same notation is used as defined in their paper,

$$
\lambda=\frac{\omega}{k}, \tilde{k}=k \tilde{L}, \tilde{L}=\frac{V_{A n} \cos \theta}{\nu_{n i}} .
$$

Note that Equation (19) can be directly obtained from Equation (3) by omitting the first term $\omega^{3}$ in Equation (3), which is unimportant at low wave frequencies.

The exact solutions to Equation (19) are straightforward,

$$
\lambda=\frac{-i \tilde{k} \pm \sqrt{4-\tilde{k}^{2}}}{2} V_{A n} \cos \theta .
$$

As is shown by Kulsrud \& Pearce (1969), the solutions become purely imaginary when $\tilde{k}>2$, which is equivalent to $k_{\|}>2 \nu_{n i} / V_{A n}$. From Equation (7a), we see $2 \nu_{n i} / V_{A n} \approx k_{c, \|}^{+}$in a mostly neutral plasma. It shows that the single-fluid approach also can capture the lower cutoff boundary.

At $\tilde{k}<2$, we express $\lambda$ as $\left(\omega_{R}+i \omega_{I}\right) / k$. Together with Equation (20), we obtain

$$
\begin{gathered}
\omega_{R}^{2}=\frac{4-\tilde{k}^{2}}{4} V_{A n}^{2} k^{2} \cos ^{2} \theta, \\
\omega_{I}=-\frac{1}{2} \tilde{k} k \cos \theta V_{A n}=-\frac{V_{A n}^{2} k^{2} \cos ^{2} \theta}{2 \nu_{n i}} .
\end{gathered}
$$

In comparison with the solutions to the two-fluid dispersion relation in the strongly coupled regime (Equation (5)), with $\tilde{k}^{2} \ll 4$ at low wave frequencies, Equation (22) coincides with Equation (5) in a weakly ionized plasma. 
The dispersion relation of the compressible modes is (Balsara 1996),

$$
\begin{aligned}
\lambda^{4} & +i \tilde{k} V_{A n} \sec \theta \lambda^{3}-\left(c_{s n}^{2}+V_{A n}^{2}\right) \lambda^{2} \\
& -i \tilde{k} V_{A n} \sec \theta c_{s n}^{2} \lambda+V_{A n}^{2} c_{s n}^{2} \cos ^{2} \theta=0 .
\end{aligned}
$$

Recall that we derived the analytical solutions to the two-fluid dispersion relation under the weak damping approximation in Section 3.1. To better capture the wave behavior beyond the weak damping limit, here we follow a different procedure called Newton's method to obtain the approximate solutions. To simplify the problem, we consider a paradigmatic case of a low- $\beta$ plasma. The approximate expressions of wave frequencies in some tractable limit cases are provided in Appendix B.

We treat the left-hand side of Equation (23) as a function $f$ and adopt the approximate roots of $f=0$ at the extreme $\beta \rightarrow 0$ as the starting point,

$$
\begin{gathered}
\lambda_{0}^{1,2}=\frac{-i \tilde{k} \sec \theta \pm \sqrt{4-\tilde{k}^{2} \sec ^{2} \theta}}{2} V_{A n} \\
\lambda_{0}^{3,4}= \pm c_{s n} \cos \theta
\end{gathered}
$$

$\lambda_{0}^{1,2}$ and $\lambda_{0}^{3,4}$ correspond to fast and slow waves, respectively. Based on $\lambda_{0}$, a better approximation is given by

$$
\lambda_{1}=\lambda_{0}-\frac{f\left(\lambda_{0}\right)}{f^{\prime}\left(\lambda_{0}\right)},
$$

where $f^{\prime}\left(\lambda_{0}\right)$ is the derivative of $f$ at $\lambda_{0}$. This one-step iteration can already converge to the actual solutions well. But the expressions of $\lambda_{1}$ can be too complicated to be illustrative. Therefore, instead of applying $\lambda_{1}$ (not shown for simplicity), we find that $\lambda_{1}$ can be further reduced at low- $\beta$ condition and becomes

$$
\begin{gathered}
\lambda_{1}^{1,2} \approx \frac{-i \tilde{k} \sec \theta \pm \sqrt{4-\tilde{k}^{2} \sec ^{2} \theta}}{2} V_{A n}, \\
\lambda_{1}^{3,4} \approx \pm c_{s n} \cos \theta-\frac{c_{s n}^{2} \tilde{k} \tan ^{2} \theta \cos \theta}{2 V_{A n}} i .
\end{gathered}
$$

We keep $\lambda_{1}^{1,2}$ the same as $\lambda_{0}^{1,2}$ since the modification is negligible. From Equation (26a), we see that fast waves become nonpropagating when $\tilde{k}>2 \cos \theta$. That is, $k>2 \nu_{n i} / V_{A n}$, in accordance with $k_{c}^{+}$given by Equation $(16 a)$.

When $\tilde{k}<2 \cos \theta$, we rewrite Equation (26) in terms of $\omega_{R}$ and $\omega_{I}$, and obtain

$$
\begin{gathered}
\omega_{R}^{2}=\frac{4-\tilde{k}^{2} \sec ^{2} \theta}{4} V_{A n}^{2} k^{2}, \\
\omega_{I}=-\frac{\tilde{k} k \sec \theta}{2} V_{A n}=-\frac{V_{A n}^{2} k^{2}}{2 \nu_{n i}}
\end{gathered}
$$

for fast waves, and

$$
\begin{gathered}
\omega_{R}^{2}=c_{s n}^{2} k^{2} \cos ^{2} \theta, \\
\omega_{I}=-\frac{c_{s n}^{2} \tilde{k} k \tan ^{2} \theta \cos \theta}{2 V_{A n}}=-\frac{c_{s n}^{2} k_{\perp}^{2}}{2 \nu_{n i}}
\end{gathered}
$$

for slow waves. In a weakly ionized medium, they are in good agreement with Equations (12) and (13) derived using the twofluid approach.
Zaqarashvili et al. (2012) argued that the appearance of the cutoff wavenumber in Alfvén waves is not a physical phenomenon under the single-fluid description. Nevertheless, the above analysis demonstrates in the strongly coupled regime, that the single-fluid approach provides the same analytical wave frequencies as those obtained using the two-fluid approach in a neutral-dominated medium. Furthermore, the different analytical procedure we applied for single-fluid MHD can inform us of the lower cutoff boundary directly from the expression of $\omega_{R}$ in the cases of Alfvén and fast waves. This indicates that the singlefluid approach is adequate to deal with turbulence damping when damping occurs in the strongly coupled regime.

In addition, we also need to point out, similar to the simplified expression of the two-fluid $\omega_{I}$ in the low- $\beta$ condition (Equation $(13 b)$ ), that we also see from the single-fluid $\omega_{I}$ (Equation (28b)) that damping is absent in the case of purely parallel propagation of slow modes. This conclusion was earlier reached through normal mode analysis in the single-fluid approach by Forteza et al. (2007), whereas the two-fluid approach by Zaqarashvili et al. (2011) and the energy equations given by Braginskii (1965) both showed a non-zero damping rate for purely parallel propagation of slow modes. Indeed, according to the general expression of $\omega_{I}$ in Equation $(11 b), \omega_{I}$ is not zero at $\theta=0$. But this does not mean that the simplified expression of $\omega_{I}$ in a low- $\beta$ medium (Equations (13b) and $(28 b))$ is invalid. First, from Equation $(11 b)$ we see that the term with $k_{\|}$is subdominant and can be safely neglected in the low- $\beta$ condition; and second, $k_{\|}$becomes more and more insignificant compared to $k_{\perp}$ toward smaller scales due to turbulence anisotropy (see next section). The above analytical damping rates will be numerically tested in Section 5 .

\section{DAMPING OF MHD TURBULENCE}

MHD turbulence is a highly nonlinear phenomenon, as a cascade of turbulent energy toward the undamped smallest scale. The remarkable feature of the GS95 model of turbulence is that the hydrodynamic-like mixing motions of the magnetic field lines in the perpendicular direction have the same timescale as that of the wave-like motions along field lines. That is, Alfvénic turbulence cascades over one wave period, $k_{\|} V_{A} \sim k_{\perp} v_{\perp}$, which is called the critical balance condition. Combining the Kolmogorov scaling for the hydrodynamic-like motions $v_{\perp} \propto k_{\perp}^{-1 / 3}$, the critical balance condition naturally leads to the scale-dependent anisotropy, $k_{\|} \sim k_{\perp}^{2 / 3}$. It cannot be overemphasized that the GS95 laws are true only in a local reference system with respect to the local configuration of magnetic field lines. The importance of the local notion was not pointed out by GS95, but demonstrated in Lazarian \& Vishniac (1999) and later numerically confirmed in Cho \& Vishniac (2000), Maron \& Goldreich (2001), and Cho et al. (2002). This specification on the reference system makes the original GS95 picture more self-consistent and entails the dependence on scales of turbulence anisotropy. For the sake of tradition and uniformity, we use the notations $k_{\|}$and $k_{\perp}$ when applying the scaling relations, but keep in mind that they should be treated as the inverse of the scales measured parallel and perpendicular to the local mean magnetic field.

The scaling relations were first introduced for trans-Alfvénic turbulence in GS95, and later generalized for different regimes of MHD turbulence including sub-Alfvénic turbulence (Lazarian \& Vishniac 1999). With the scale-dependent anisotropy of MHD turbulence taken into account, the above linear analysis 
of wave damping can be used to set the damping scales of MHD turbulence. We next briefly describe the cascading of MHD turbulence and list the corresponding damping scales derived in Paper I.

\subsection{Alfvén Modes}

Alfvén modes cascade independently of compressible modes and have cascading rates of (GS95, Lazarian 2006)

$$
\begin{gathered}
\tau_{\text {cas }}^{-1}=k^{2 / 3} L^{-1 / 3} V_{L}, \quad l_{A}<1 / k<L, \\
\tau_{\text {cas }}^{-1}=k_{\perp}^{2 / 3} L^{-1 / 3} V_{L}, \quad 1 / k<l_{A},
\end{gathered}
$$

for super-Alfvénic turbulence, i.e., $M_{A}=V_{L} / V_{A}>1$. Here $l_{A}=L M_{A}^{-3}$ is the scale where turbulence anisotropy starts to develop and increases toward smaller scales. At scales smaller than $l_{A}$, the parallel and perpendicular components of the wavevector with respect to the local direction of the magnetic field are related by

$$
k_{\|} \sim l_{A}^{-1}\left(k_{\perp} l_{A}\right)^{2 / 3} .
$$

From Equation (29) we see that different scaling relations apply in different ranges of scales as the statistical description for motions on large scales can only be applicable to motions perpendicular to the local magnetic field below the scale $l_{A}$. At $1 / k=l_{A}$, the turbulent energy becomes in equipartition with the magnetic energy, and the isotropic scaling as on larger scales still applies as assumed in the original Goldreich \& Sridhar (1995) formulation of MHD turbulence. Toward smaller scales, magnetic effects become more prominent. As a result, the cascade perpendicular to the local direction of the magnetic field dominates and changes the scaling to be anisotropic. Note that as the damping scale is usually far smaller than $l_{A}$, its perpendicular and parallel components with respect to the magnetic field conform to the anisotropic scaling.

Sub-Alfvénic turbulence has $M_{A}<1$ and a cascading rate (Lazarian \& Vishniac 1999)

$$
\begin{aligned}
& \tau_{\text {cas }}^{-1}=\frac{\left(k v_{l}\right)^{2}}{\omega_{A}}=\frac{k^{2} V_{L}^{2}}{k_{\perp} V_{A}}, \quad l_{\text {tr }}<1 / k<L, \\
& \tau_{\text {cas }}^{-1}=v_{l} / l_{\perp}=k_{\perp}^{\frac{2}{3}} L^{-\frac{1}{3}} V_{L} M_{A}^{\frac{1}{3}}, \quad 1 / k<l_{\text {tr }},
\end{aligned}
$$

where $l_{\text {tr }}=L M_{A}^{2}$ is the transition scale from weak to strong turbulence. Weak turbulence only evolves in the perpendicular direction with $k_{\perp}$ increasing during the cascade, while strong turbulence has the scaling relation

$$
k_{\|} \sim L^{-1}\left(k_{\perp} L\right)^{2 / 3} M_{A}^{4 / 3} .
$$

The damping scale of turbulence can be analytically determined by setting $\left|\omega_{I}\right| \sim \tau_{\text {cas }}^{-1}$. With both damping effects included, the general expression of the damping scale is

$$
\begin{aligned}
& k_{\mathrm{dam}, \|}=\frac{-\left(\nu_{n}+\frac{V_{A i}^{2}}{\nu_{i n}}\right)+\sqrt{\left(\nu_{n}+\frac{V_{A i}^{2}}{\nu_{i n}}\right)^{2}+\frac{8 V_{A} \nu_{n} l_{A}}{\xi_{n}}}}{2 \nu_{n} l_{A}},
\end{aligned}
$$

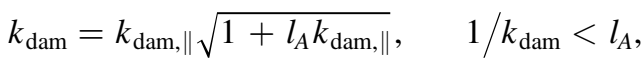

for super-Alfvénic turbulence, and

$$
\begin{aligned}
& k_{\mathrm{dam}, \|}=\frac{-\left(\nu_{n}+\frac{V_{A i}^{2}}{\nu_{i n}}\right)+\sqrt{\left(\nu_{n}+\frac{V_{A i}^{2}}{\nu_{i n}}\right)^{2}+\frac{8 V_{A} \nu_{n} L M_{A}^{-4}}{\xi_{n}}}}{2 \nu_{n} L M_{A}^{-4}},
\end{aligned}
$$

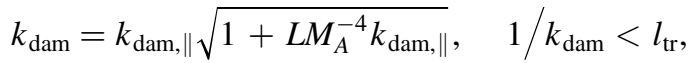

for sub-Alfvénic turbulence. In most situations, only the dominant damping effect needs to be considered. In the case when damping is dominated by ion-neutral collisions, from Equations (5b), (29b), (30), (31b), and (32), we obtain

$$
k_{\mathrm{dam}}=\left(\frac{2 \nu_{n i}}{\xi_{n}}\right)^{\frac{3}{2}} L^{\frac{1}{2}} V_{L}^{-\frac{3}{2}}
$$

for super-Alfvénic turbulence when $k_{\mathrm{dam}}^{-1}<l_{A}$, and

$$
k_{\mathrm{dam}}=\left(\frac{2 \nu_{n i}}{\xi_{n}}\right)^{\frac{3}{2}} L^{\frac{1}{2}} V_{L}^{-\frac{3}{2}} M_{A}^{-\frac{1}{2}}
$$

for sub-Alfvénic turbulence when $k_{\mathrm{dam}}^{-1}<l_{\text {tr }}$.

By applying the scaling relations Equations (30) and (32) to the decoupling scales of the Alfvén modes in Table 1, we find that $k_{\mathrm{dec}, n i}$ is directly related with $k_{\mathrm{dam}}$ in the above equations by

$$
k_{\mathrm{dec}, n i}=\left(\frac{2}{\xi_{n}}\right)^{-\frac{3}{2}} k_{\mathrm{dam}}
$$

for both super- and sub-Alfvénic turbulence. And $k_{\mathrm{dec}, \text { in }}$ takes the form

$$
k_{\mathrm{dec}, i n}=\left(\frac{\nu_{i n}}{V_{A i}}\right)^{\frac{3}{2}} L^{\frac{1}{2}} M_{A}^{-\frac{3}{2}}
$$

in super-Alfvénic turbulence, and

$$
k_{\mathrm{dec}, i n}=\left(\frac{\nu_{i n}}{V_{A i}}\right)^{\frac{3}{2}} L^{\frac{1}{2}} M_{A}^{-2}
$$

in sub-Alfvénic turbulence. Here we use the perpendicular component $k_{\perp}$ to represent total $k$, since we assume that the decoupling and damping scales are sufficiently small where turbulence anisotropy is prominent, which is a common occurrence in astrophysical environments.

When neutral-viscous damping plays a more important role, a combination of Equations (9), (29b), (30), (31b), and (32) yields

$$
k_{\mathrm{dam}}=\nu_{n}^{-\frac{3}{4}} L^{-\frac{1}{4}} V_{L}^{\frac{3}{4}}, \quad 1 / k_{\mathrm{dam}}<l_{A},
$$

for super-Alfvénic turbulence, and

$$
k_{\mathrm{dam}}=\nu_{n}^{-3 / 4} L^{-1 / 4} V_{L}^{3 / 4} M_{A}^{1 / 4}, \quad 1 / k_{\mathrm{dam}}<l_{\mathrm{tr}},
$$

for sub-Alfvénic turbulence. The assumption made here is that the mean free path of neutral-neutral collisions $l_{n}$ is relatively small compared with $l_{A}$ in super-Alfvénic turbulence and $L$ in sub-Alfvénic turbulence, which is reasonable under most ISM conditions.

It is instructive to compare the cutoff wavenumbers derived from linear MHD waves and the damping scales of MHD turbulence. Due to the critical balance, $\left|\omega_{R}\right|=\tau_{\text {cas }}^{-1}$ holds for Alfvénic turbulence. Consequently, the cutoff condition 
Table 2

Cutoff Boundaries of MHD Modes

\begin{tabular}{ccccc}
\hline \hline & \multicolumn{2}{c}{$k_{c}^{+}$} & & $k_{c}^{-}$ \\
\hline Alfvén & Super & IN & Equation (35)* & $\left(\frac{\nu_{\text {in }}}{2 V_{A i}}\right)^{\frac{3}{2}} L^{\frac{1}{2}} M_{A}^{-\frac{3}{2}}$ \\
& & NV & Equation (40) & \\
& Sub & IN & Equation (36) & $\left(\frac{\nu_{\text {in }}}{2 V_{A i}}\right)^{\frac{3}{2}} L^{\frac{1}{2}} M_{A}^{-2}$ \\
& & NV & Equation (41) & \\
& & &
\end{tabular}

\begin{tabular}{lccc}
\hline Fast & Equation $(16 a)$ & Equation $(16 b)$ \\
\hline Slow & Super & $\left(\frac{2 \nu_{n i}}{c_{s} \xi_{n}}\right)^{\frac{3}{4}} L^{-\frac{1}{4}} M_{A}^{\frac{3}{4} *}$ & $\left(\frac{\nu_{i n}}{2 c_{s i}}\right)^{\frac{3}{2}} L^{\frac{1}{2}} M_{A}^{-\frac{3}{2}}$ \\
& Sub & $\left(\frac{2 \nu_{n i}}{c_{s} \xi_{n}}\right)^{\frac{3}{4}} L^{-\frac{1}{4}} M_{A}{ }^{*}$ & $\left(\frac{\nu_{i n}}{2 c_{s i}}\right)^{\frac{3}{2}} L^{\frac{1}{2}} M_{A}^{-2}$ \\
\hline
\end{tabular}

$\left|\omega_{R}\right|=\left|\omega_{I}\right|$ of MHD waves is equivalent to the damping condition $\tau_{\text {cas }}^{-1}=\left|\omega_{I}\right|$ of MHD turbulence. Note that the wave propagation direction, $\cos \theta=k_{\|} / k$, follows the scaling relations given by Equations (30) and (32), and is scale-dependent. By taking this into account, the $k_{c}^{+}$corresponding to Equations (7a) and (10) are fully consistent with $k_{\mathrm{dam}}$ expressed by Equations (35), (36), (40), and (41). In Table 2, we summarize the expressions for the cutoff boundaries $k_{c}^{ \pm}$of the three turbulence modes by taking the scaling relations of MHD turbulence into account. "IN" and "NV" represent ionneutral collisional and neutral-viscous damping. The asterisks indicate that $k_{c}^{+}$coincides with $k_{\mathrm{dam}}$ in corresponding situations.

Regarding the relation between the cutoff boundaries and decoupling scales, from Equations (37)-(39) and Table 2, we deduce

$$
\begin{gathered}
k_{c}^{+}=\left(\frac{2}{\xi_{n}}\right)^{\frac{3}{2}} k_{\mathrm{dec}, n i}, \\
k_{c}^{-}=2^{-\frac{3}{2}} k_{\mathrm{dec}, i n}
\end{gathered}
$$

in the case of ion-neutral collisional damping. The difference between them is independent of turbulence properties.

\subsection{Compressible Modes}

Cho \& Lazarian (2003) found fast modes form in both highand low- $\beta$ media and follow a cascade similar to the one in acoustic turbulence. The cascading rate of fast modes is (Cho \& Lazarian 2003; Yan \& Lazarian 2004)

$$
\begin{aligned}
& \tau_{\text {cas }}^{-1}=\left(\frac{k}{l_{A}}\right)^{\frac{1}{2}} \frac{V_{A}^{2}}{V_{f}}, \quad M_{A}>1, \\
& \tau_{\text {cas }}^{-1}=\left(\frac{k}{L}\right)^{\frac{1}{2}} \frac{V_{L}^{2}}{V_{f}}, \quad M_{A}<1,
\end{aligned}
$$

where $V_{f}$ is the phase speed of fast modes. It takes the form

$$
V_{f}=\sqrt{\frac{1}{2}\left(c_{s}^{2}+V_{A}^{2}\right)+\frac{1}{2} \sqrt{\left(c_{s}^{2}+V_{A}^{2}\right)^{2}-4 c_{s}^{2} V_{A}^{2} \cos ^{2} \theta}}
$$

in the strongly coupled regime, and

$$
V_{f}=\sqrt{\frac{1}{2}\left(c_{s i}^{2}+V_{A i}^{2}\right)+\frac{1}{2} \sqrt{\left(c_{s i}^{2}+V_{A i}^{2}\right)^{2}-4 c_{s i}^{2} V_{A i}^{2} \cos ^{2} \theta}}
$$

in the weakly coupled regime. The transition of $V_{f}$ takes place at $k_{\mathrm{dec}, \text { in }}$ if the turbulence survives damping. Fast modes cascade radially, so the wave propagation direction can be considered fixed during the cascade. The intersection scale between $\tau_{\text {cas }}^{-1}$ with $V_{f}$ from Equation (44) and $\left|\omega_{I}\right|$ (Equation (11b)) corresponds to the damping scale

$$
\begin{aligned}
& k_{\mathrm{dam}}=l_{A}^{-\frac{1}{3}}\left(\frac{2 \nu_{n i} V_{A}^{2}\left(c_{s}^{2}+V_{A}^{2}-2 V_{f}^{2}\right)}{V_{f}\left[\xi_{n} V_{A}^{2}\left(c_{s}^{2}-V_{f}^{2}\right)+\xi_{i} c_{s}^{2} V_{f}^{2}\right]}\right)^{\frac{2}{3}}, \\
& k_{\mathrm{dam}}=L^{-\frac{1}{3}}\left(\frac{2 \nu_{n i} V_{L}^{2}\left(c_{s}^{2}+V_{A}^{2}-2 V_{f}^{2}\right)}{V_{f}\left[\xi_{n} V_{A}^{2}\left(c_{s}^{2}-V_{f}^{2}\right)+\xi_{i} c_{s}^{2} V_{f}^{2}\right]}\right)^{\frac{2}{3}},
\end{aligned}
$$

for super- and sub-Alfvénic turbulence, respectively. In a low- $\beta$ plasma, the above expressions can be written as

$$
\begin{gathered}
k_{\mathrm{dam}}=\left(\frac{2 \nu_{n i}}{\xi_{n}}\right)^{2 / 3} l_{A}^{-1 / 3} V_{A}^{-2 / 3}, \quad M_{A}>1, \\
k_{\mathrm{dam}}=\left(\frac{2 \nu_{n i}}{\xi_{n}}\right)^{2 / 3} V_{L}^{4 / 3} L^{-1 / 3} V_{A}^{-2}, \quad M_{A}<1 .
\end{gathered}
$$

By comparing the damping scale with the neutral-ion decoupling scale (Table 1), we find

$$
\begin{gathered}
\frac{k_{\mathrm{dam}}}{k_{\mathrm{dec}, n i}}=\left(\frac{2}{\xi_{n}}\right)^{\frac{2}{3}}\left(k_{\mathrm{dec}, n i} l_{A}\right)^{-\frac{1}{3}}, \quad M_{A}>1, \\
\frac{k_{\mathrm{dam}}}{k_{\mathrm{dec}, n i}}=\left(\frac{2}{\xi_{n}}\right)^{\frac{2}{3}}\left(k_{\mathrm{dec}, n i} L\right)^{-\frac{1}{3}} M_{A}^{\frac{4}{3}}, \quad M_{A}<1 .
\end{gathered}
$$

Since $\left(k_{\mathrm{dec}, n i} l_{A}\right)^{-1}$ and $\left(k_{\mathrm{dec}, n i} L\right)^{-1}$ are usually much smaller than $1, k_{\mathrm{dam}}<k_{\mathrm{dec}, n i}$ is a common occurrence. This means that the damping of fast modes takes place before neutral-ion decoupling. It is worth mentioning that since the cutoff scale is always smaller than the neutral-ion decoupling scale for fast modes (Equation (18a)), the cutoff does not play a role in the damping of fast modes.

Slow modes cascade passively and have the same $\tau_{\text {cas }}^{-1}$ as Alfvén modes (GS95). According to the critical balance, the damping condition $\tau_{\text {cas }}^{-1}=\left|\omega_{I}\right|$ is equivalent to $k_{\|} V_{A}=\left|\omega_{I}\right|$, while the cutoff condition $\left|\omega_{R}\right|=\left|\omega_{I}\right|$ gives $k_{\|} c_{S}=\left|\omega_{I}\right|$. In a low- $\beta$ medium, $\tau_{\text {cas }}^{-1}$ is always larger than $\left|\omega_{R}\right|$ on a certain scale. This means that the cutoff of slow waves happens before the equilibrium between $\tau_{\text {cas }}^{-1}$ and $\left|\omega_{I}\right|$ is reached. Therefore the damping scale of slow modes depends on the cutoff scale $1 / k_{c}^{+}$ (see Table 2 for the expressions). On the other hand, slow modes dissipate subsequently after Alfvén modes are damped out, so their damping scale should not be smaller than that of Alfvén modes. Accordingly, in combination with 
Equations (35) and (36), we have $k_{\text {dam }}$ of slow modes as

$$
k_{\mathrm{dam}}=\min \left[\left(\frac{2 \nu_{n i}}{c_{S} \xi_{n}}\right)^{\frac{3}{4}} L^{-\frac{1}{4}} M_{A}^{\frac{3}{4}},\left(\frac{2 \nu_{n i}}{\xi_{n}}\right)^{\frac{3}{2}} L^{\frac{1}{2}} V_{L}^{-\frac{3}{2}}\right]
$$

when $1 / k_{\text {dam }}<l_{A}$ in super-Alfvénic turbulence, and

$$
k_{\mathrm{dam}}=\min \left[\left(\frac{2 \nu_{n i}}{c_{s} \xi_{n}}\right)^{\frac{3}{4}} L^{-\frac{1}{4}} M_{A},\left(\frac{2 \nu_{n i}}{\xi_{n}}\right)^{\frac{3}{2}} L^{\frac{1}{2}} V_{L}^{-\frac{3}{2}} M_{A}^{-\frac{1}{2}}\right]
$$

when $1 / k_{\text {dam }}<l_{\text {tr }}$ in sub-Alfvénic turbulence. In fact, the ratio between the two terms, $k_{c}^{+}$of the slow modes and $k_{\text {dam }}$ of Alfvén modes in Equations (49) and (50) reveals

$$
\begin{gathered}
\frac{k_{c, s}^{+}}{k_{\mathrm{dam}, A}}=\left[\left(\frac{\xi_{n}}{2}\right)\left(\frac{V_{A}}{c_{s}}\right)\left(k_{\mathrm{dec}, n i, \|} l_{A}\right)^{-1}\right]^{\frac{3}{4}}, \quad M_{A}>1, \\
\frac{k_{c, s}^{+}}{k_{\mathrm{dam}, A}}=\left[\left(\frac{\xi_{n}}{2}\right)\left(\frac{V_{A}}{c_{s}}\right)\left(k_{\mathrm{dec}, n i, \|} L\right)^{-1} M_{A}^{4}\right]^{\frac{3}{4}}, \quad M_{A}<1,
\end{gathered}
$$

where $\quad k_{\mathrm{dec}, n i, \|}=\nu_{n i} / V_{A}$. The terms $\quad\left(k_{\mathrm{dec}, n i, \|} l_{A}\right)^{-1}$ and $\left(k_{\mathrm{dec}, n i, \|} L\right)^{-1}$ are usually much smaller than 1 . If the $\beta$ value of the medium is not considerably small, $k_{c, s}^{+}<k_{\mathrm{dam}, A}$ stands. Thus we can safely take $k_{\text {dam }}=k_{c}^{+}$for slow modes in most cases.

Slow modes have the same relation between $k_{c}^{-}$and $k_{\mathrm{dec}, \text { in }}$ as Alfvén modes (see Equation (42b)). We then examine the relation between $k_{c}^{+}$and $k_{\mathrm{dec}, n i}$. The neutral-ion decoupling condition $c_{s n} k=\nu_{n i}$ can be written as

$$
\frac{\xi_{n} c_{s n}^{2} k^{2}}{2 \nu_{n i}}=\frac{\xi_{n} c_{s n} k}{2}
$$

while the cutoff condition is (see Equation (13))

$$
\frac{\xi_{n} c_{s}^{2} k_{\perp}^{2}}{2 \nu_{n i}}=c_{s} k_{\|}
$$

Under the condition of strong turbulence anisotropy, i.e., $k \sim k_{\perp}$ and $k_{\perp} \gg k_{\|}$, we see that the cutoff condition can be reached on a larger scale than the neutral-ion decoupling scale. Thus slow modes fall into a different situation from fast and Alfvén modes (see Equations (18a) and (42a)) and can have $k_{c}^{+}<k_{\mathrm{dec}, n i}$. But in terms of damping, due to $k_{\mathrm{dam}}=k_{c}^{+}$, slow modes are similar to fast modes and are also damped out when neutrals and ions are strongly coupled together.

Comparing this section with Section 3, we can see that the main difference between the damping processes of linear MHD waves and MHD turbulence is the consideration of scaledependent anisotropy, which depends on the turbulence properties. For our study on turbulence damping of Alfvén and slow modes, a fixed wave propagation direction, e.g., purely parallel or perpendicular propagation, has no physical significance. Furthermore, as regards scaling estimations, e.g., evaluation of the damping scales, turbulence anisotropy is the essential physical ingredient. Ignorance of it can lead to severely wrong conclusions in related astrophysical applications.
Table 3

Parameters Used for Different Phases of Partially Ionized ISM and SC

\begin{tabular}{lccccc}
\hline \hline & WNM & CNM & MC & DC & SC \\
\hline$n_{\mathrm{H}}\left[\mathrm{cm}^{-3}\right]$ & 0.4 & 30 & 300 & $10^{4}$ & $4.2 \times 10^{12}$ \\
$n_{e} / n_{\mathrm{H}}$ & 0.1 & $10^{-3}$ & $10^{-4}$ & $10^{-6}$ & $1.78 \times 10^{-2}$ \\
$T[\mathrm{~K}]$ & 6000 & 100 & 20 & 10 & 6220 \\
$B[\mu \mathrm{G}]$ & 8.66 & 8.66 & 8.66 & 86.6 & $6.96 \times 10^{7}$ \\
$\beta$ & 0.22 & 0.23 & 0.20 & 0.03 & 0.03 \\
$M_{A}$ & 0.4 & 2.9 & 9.2 & 5.3 & 0.4 \\
\hline
\end{tabular}

\section{NUMERICAL TESTS IN DIFFERENT PHASES OF THE PARTIALLY IONIZED ISM AND SC}

The generality of the two-fluid approximation allows us to apply our results to a wide variety of astrophysical situations. We next apply the analysis on turbulence damping in different phases of partially ionized ISM. To avoid confusion, we stress that by numerical tests, we mean numerically solving the full dispersion relations with given physical parameters, but not performing any MHD simulations.

Table 3 lists the typical physical parameters for the warm neutral medium (WNM), cold neutral medium (CNM), MC, and dense core (DC) in an MC. The parameters $n_{\mathrm{H}}, n_{e} / n_{\mathrm{H}}$, and $T$ are taken from Lazarian et al. (2004). We assume that temperatures are equal for all species. Magnetic field strengths are $\sqrt{3} \times$ the half maximum values of the Zeeman measurements from Figure 1 in Crutcher et al. (2010) as the total strength of the three-dimensional magnetic field. We use the typical driving condition of turbulence by assuming supernova explosions as the main source of energy injection of the ISM turbulence (Spitzer 1978; Bykov \& Toptygin 2001),

$$
L=30 \mathrm{pc}, \quad V_{L}=10 \mathrm{~km} \mathrm{~s}^{-1} .
$$

The Alfvénic Mach number $M_{A}$ can then be determined from the velocity perturbation $V_{L}$ at the injection scale $L$ as $V_{L} / V_{A}$. In addition, we adopt the drag coefficient as $\gamma_{d}=3.5 \times$ $10^{13} \mathrm{~cm}^{3} \mathrm{~g}^{-1} \mathrm{~s}^{-1}$ from Draine et al. (1983) ${ }^{7}$, and the collisional cross-section for neutrals $\sigma_{n n} \sim 10^{-14} \mathrm{~cm}^{2}$ according to Vranjes $\&$ Krstic (2013). The masses of ions and neutrals are $m_{i}=m_{n}=m_{\mathrm{H}}$ for the WNM and CNM, and $m_{i}=29 m_{\mathrm{H}}$, $m_{n}=2.3 m_{\mathrm{H}}$ for the other environments (Shu 1992).

Observations confirm that MHD waves are not only found in the ISM, but are also ubiquitous in the solar atmosphere (e.g., De Pontieu et al. 2007, 2012). The damping processes of MHD waves in the partially ionized solar atmosphere, i.e., photosphere and chromosphere, have been widely suggested as a heating mechanism of the external solar atmosphere (e.g., Osterbrock 1961; Hollweg 1986; Narain \& Ulmschneider 1996; Goodman 2000, 2001). Thus we also extend our analysis to the SC environment. In reality, the physical parameters vary with altitude from the photosphere level. For the sake of simplicity, we neglect the vertical variation, but consider a toy model in a homogeneous medium with all parameters taken at a median height. This simplified analysis serves as a paradigmatic case of damping in a chromosphere-like environment. A more sophisticated model may be required when making comparisons to observational data.

\footnotetext{
This constant $\gamma_{d}$ applies under conditions like MCs where the relative velocities between neutrals and ions are relatively low and the effective crosssection of ion-neutral collisions is much larger than the geometric cross-section (see Shu 1992).
} 
We adopt model C for a quiet Sun in Vernazza et al. (1981) at a height of $1280 \mathrm{~km}$ and only consider hydrogen. The magnetic field strength is obtained by assuming $B=$ $B_{\mathrm{ph}}\left(\rho / \rho_{\mathrm{ph}}\right)^{0.3}$ (Leake \& Arber 2006). With $B_{\mathrm{ph}}=1.5 \times 10^{3}$ $\mathrm{G}$ and $\rho_{\mathrm{ph}}=2.73 \times 10^{-7} \mathrm{~g} \mathrm{~cm}^{-3}$ at the photospheric level used, we obtain $B=69.6 \mathrm{G}$. For the ion-neutral collisional cross-section, we use the value $\left(\approx 1 \times 10^{-14} \mathrm{~cm}^{2}\right)$ proposed by Vranjes \& Krstic (2013). Their approach, containing energy dependence and quantum effects, yields a collisional crosssection two orders of magnitude larger than that obtained from the hard sphere model (Braginskii 1965). Then drag coefficient can be calculated with the expression (Braginskii 1965; Soler et al. 2013a),

$$
\gamma_{d}=\frac{1}{2 m_{\mathrm{H}}} \sqrt{\frac{16 k_{B} T}{\pi m_{\mathrm{H}}}} \sigma_{i n}
$$

In addition, we use the height of the chromosphere as the injection scale $L=2500 \mathrm{~km}$, and assume a turbulent velocity at $L$ as $V_{L}=30 \mathrm{~km} \mathrm{~s}^{-1}$ based on the velocity amplitude measurement of chromospheric waves by Okamoto \& De Pontieu (2011). The parameters are also listed in Table 3.

According to the values of $\beta$ listed in Table 3 , in the following comparisons with numerical results we will use the simplified analytical results introduced in previous sections for dealing with low- $\beta$ media.

\subsection{Alfvén Modes}

We adopt the same turbulence driving condition as in Equation (54) for the Alfvén, fast, and slow modes. Figure 1(a) illustrates the damping of the sub-Alfvénic turbulence in the WNM. The cascading rate is given by Equation $(31 b)$. Note that the scales shown are within the strong turbulence regime. We show the damping rates obtained by numerically solving the two-fluid dispersion relations with only ion-neutral collisional damping (Equation (3)) and both ionneutral collisional and neutral-viscous damping (see Paper I, Equation (19); here we provide the derivation in Appendix C). In this case, ion-neutral collisions and neutral viscosity have comparable damping efficiencies in the strongly coupled regime, so the general expression of $k_{\mathrm{dam}}$ (Equation (34)) applies. The analytical damping rate is from Equation ( $4 b)$, which coincides with the numerical results at both small and large wavenumbers, but underestimates the numerical result at intermediate wavenumbers when $\left|\omega_{I}\right|$ and $\left|\omega_{R}\right|$ are of the same order. The singlefluid damping rate is given by Equation (22b), which traces the numerical one well at small wavenumbers. The numerical solutions do not exhibit a nonpropagating interval with $\omega_{R}=0$.

Figures 1(b) and (c) display the damping of super-Alfvénic turbulence in the CNM and DC. The solutions with both damping effects are in full coincidence with those considering only ion-neutral collisional damping due to negligible viscous damping. Accordingly, $k_{\mathrm{dam}}$ for ion-neutral collisional damping (Equation (35)) applies, which coincides with the lower cutoff boundary $k_{c}^{+}$. By comparison to numerical results, we are convinced that the single-fluid approach can provide a good approximation of the actual damping rate down to the damping scale (or cutoff boundary) of Alfvén modes. The purely imaginary solutions are omitted from the numerical results, corresponding to the cutoff regions.

Figure 1(d) shows the damping of Alfvén modes in the subAlfvénic SC. The filled circles represent the analytical damping rate including both damping effects (see Paper I, equation (21b)),

$$
\left|\omega_{I}\right|=\frac{\left[\tau_{\nu}^{-1}\left(\tau_{\nu}^{-1}+(1+\chi) \nu_{n i}\right)+k^{2} \cos ^{2} \theta V_{A i}^{2}\right] \chi \nu_{n i}}{2\left[k^{2} \cos ^{2} \theta V_{A i}^{2}+\chi \tau_{\nu}^{-1} \nu_{n i}+\left(\tau_{\nu}^{-1}+(1+\chi) \nu_{n i}\right)^{2}\right]} .
$$

Obviously neutral viscosity dominates over ion-neutral collisions in damping Alfvén modes. It meets the prediction in Paper I that neutral viscosity can play a more important role in damping subAlfvénic turbulence than super-Alfvénic turbulence. The exact criteria for determining the relative importance between neutral viscosity and ion-neutral collisions in damping super- and subAlfvénic turbulence can be found in Equation (31) in Paper I. As in the case of the WNM, the general expression by Equation (34) including both damping effects can give a good approximation of $k_{\mathrm{dam}}$. But here we present $k_{\mathrm{dam}}$ with a simpler form (Equation (41)) derived from viscous damping alone as an approximate $k_{\mathrm{dam}}$. We see in the SC condition, owing to the existence of neutral viscosity, that the damping scale is considerably larger than that predicted by ion-neutral collisional damping, and is also larger than the neutral-ion decoupling scale. It shows that not only ionneutral collisions, but neutral viscosity should also be considered as an important mechanism in damping Alfvén modes.

In addition to the damping rate, the propagating component of wave frequency $\left|\omega_{R}\right|$ is also present in Figure 2 as an example in the case of Alfvén modes in MCs. The same symbols are used as in Figure 1, except that the dashed line and open squares are the numerical and analytical (Equation (5a) and $(6 a))\left|\omega_{R}\right|$, respectively. Note that as pointed out earlier in Section 4, due to the critical balance, $\left|\omega_{R}\right|$ in the strongly coupled regime coincides with the cascading rate. We find that $\left|\omega_{R}\right|$ starts to decay at $k_{\mathrm{dec}, n i}$, and is cut off at $k_{c}^{+}$. Then $\left|\omega_{R}\right|$ arises again at $k_{c}^{-}$, but is only fully resumed after reaching $k_{\mathrm{dec}, i n}$. Thus the region $\left[k_{\mathrm{dec}, n i}, k_{\mathrm{dec}, i n}\right]$ contains the cutoff zone $\left[k_{c}^{+}, k_{c}^{-}\right]$. Different from wave damping, which has a damping rate insensitive to decoupling scales, the propagating behavior of waves depends on the properties of fluid coupling, and can only be studied outside the $\left[k_{\mathrm{dec}, n i}, k_{\mathrm{dec}, i n}\right]$ region.

\subsection{Fast Modes}

Figures 3(a)-(d) display the comparison between the analytical and numerical damping rates of fast modes under different ISM conditions and the SC. The cascading rate is from Equation (43), with the wave propagation angle fixed at $45^{\circ}$. The analytical two-fluid (Equations (12b) and (6b)) and single-fluid (Equation (27b)) damping rates are in excellent agreement with the numerical results.

Both $\left|\omega_{R}\right|$ and $\left|\omega_{I}\right|$ are shown in Figure 4 for fast modes in MCs. The numerical $\left|\omega_{R}\right|$ is obtained by numerically solving the dispersion relation (Equation (51) in Soler et al. 2013a). The analytical $\left|\omega_{R}\right|$ is from Equations (12a) and (14). Similar to the case of Alfvén modes, the cutoff interval is contained within $\left[k_{\mathrm{dec}, n i}, k_{\mathrm{dec}, i n}\right]$, and only outside $\left[k_{\mathrm{dec}, n i}, k_{\mathrm{dec}, i n}\right]$ do fast waves have a phase speed equal to the Alfvén speed.

Under all the conditions presented, fast modes are damped out when neutrals and ions are strongly coupled, i.e., $k_{\mathrm{dam}}<k_{\mathrm{dec}, n i}$. In particular, fast modes in the WNM are the most severely damped among the ISM phases. The WNM has a much lower total density than other ISM conditions, which leads to a faster wave phase speed and lower neutral-ion collisional frequency. Accordingly, fast modes in the WNM have a lower cascading rate (Equation (43)) but a higher 


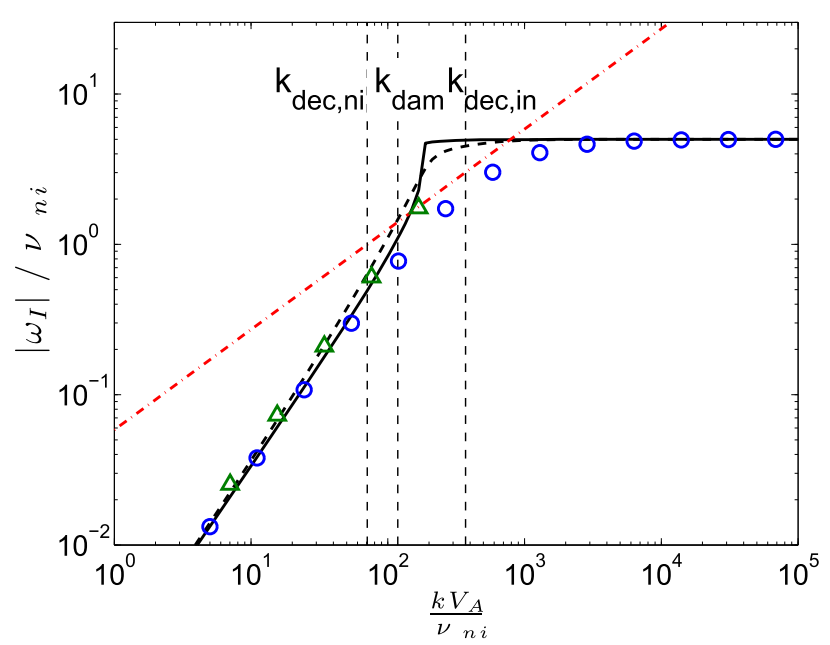

(a) WNM

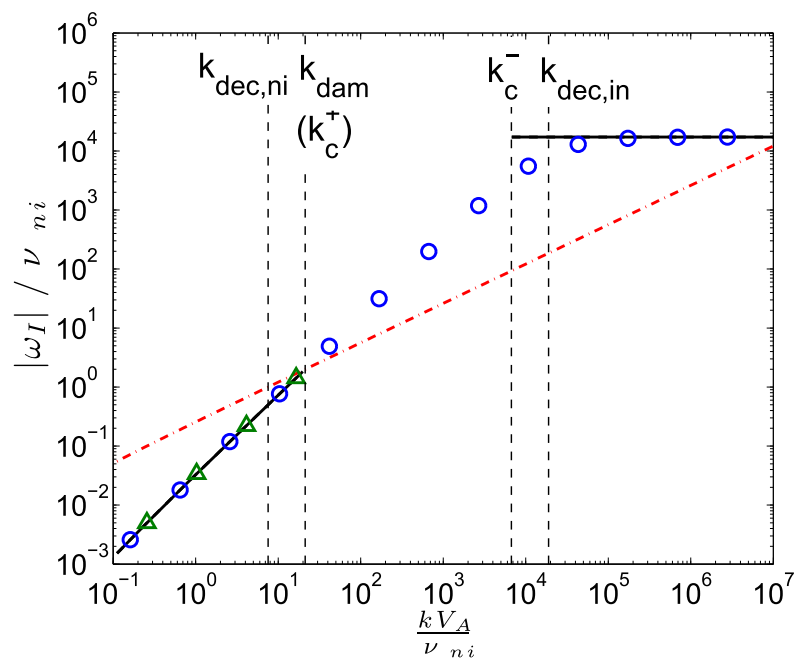

(c) $\mathrm{DC}$

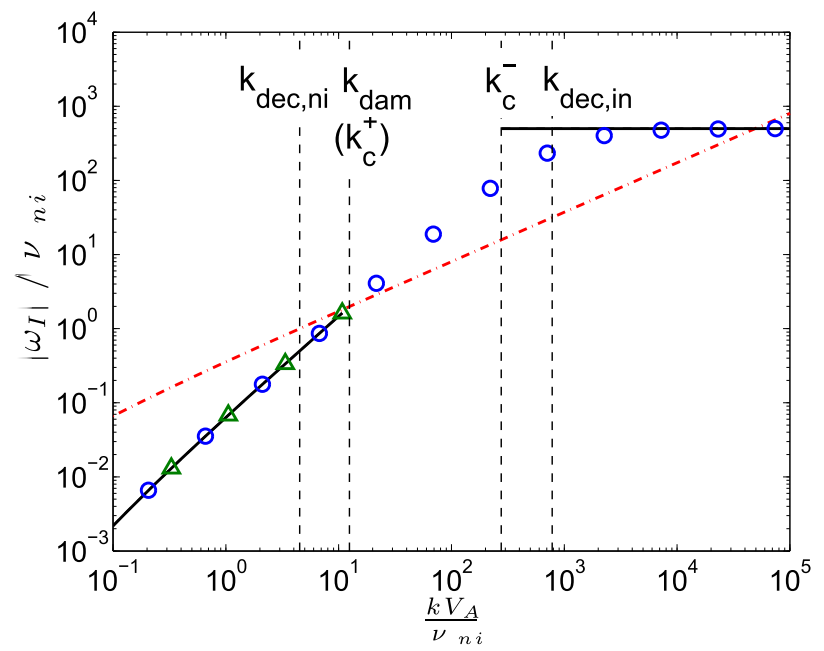

(b) $\mathrm{CNM}$

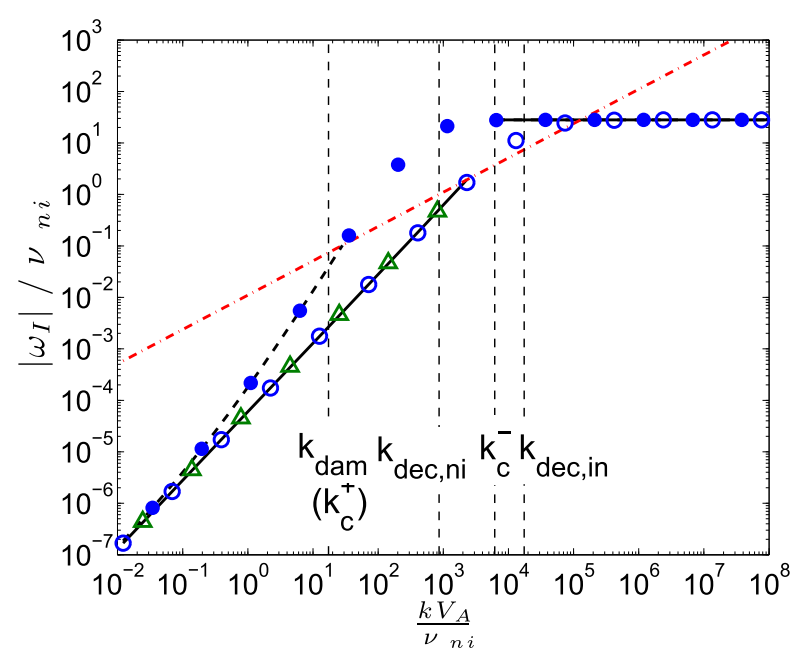

(d) $\mathrm{SC}$

Figure 1. $\left|\omega_{I}\right| / \nu_{n i}$ vs. $k V_{A} / \nu_{n i}$ of Alfvén modes in various environments. Solid lines are numerical damping rates of ion-neutral collisional damping (Equation (3)) Dashed lines are numerical damping rates including both ion-neutral collisional and viscous damping effects (Equation (19) in Paper I). Open circles are analytical damping rates corresponding to ion-neutral collisional damping (Equation (4b)). Triangles show the same as open circles, but for a single-fluid approach (Equation (22b)). The filled circles in (d) show the analytical damping rate considering both damping effects (Equation (56)). Dashed-dotted lines are the cascading rates of Alfvén modes (Equations (29b) and (31b)). They intersect with the damping rates at $k_{\mathrm{dam}}$. The analytical wavenumbers $k_{\mathrm{dec}, n i}, k_{\mathrm{dec}, i n}, k_{\mathrm{dam}}$, and $k_{c}^{ \pm}$are denoted by vertical dashed lines.

damping rate (see the discussion in Section 3.1). This means that the intersection between the two rates can take place at a large scale. In the SC, the high wave phase speed comes from the strong magnetic field strength and leads to a low cascading rate. Although a large ion density yields a high neutral-ion collisional frequency and a low damping rate (Equation (12b)), it can still exceed the substantially slow cascade on a large scale. Therefore fast modes in the SC are also severely damped. The vertical dashed lines indicate the damping scale given by Equation (47). This shows that the approximate $k_{\mathrm{dam}}$ at the low- $\beta$ limit is consistent with numerical results. In fact, according to Equation (46), $k_{\text {dam }}$ has a dependence on the wave propagation direction. Figure 5 presents the damping scale given by Equation (46b) as a function of $\theta$ in the WNM as an example. The damping scale in other cases shows similar results. We find that, despite the existence of anisotropy, the dependence of $k_{\mathrm{dam}}$ on $\theta$ is so weak that a constant damping scale can be applied. This leads to the conclusion that the energy distribution of fast modes remains isotropic over all existing scales.

\subsection{Slow Modes}

Figures 6(a)-(d) show the results for slow modes. The analytical damping rate for the two-fluid description is given by Equation (13b) for $k<k_{c}^{+}$and Equation (6b) for $k>k_{c}^{-}$, and the single-fluid damping rate is from Equation $(28 b)$. The analytical $\left|\omega_{R}\right|$ from Equation (13a) for $k<k_{c}^{+}$and Equation (15) for $k>k_{c}^{-}$is also shown in Figure 7, in the case of an MC. From Figure 7, we find that, similar to other wave modes at high wave frequencies, although $\omega_{R}$ re-emerges at the cutoff boundary $k_{c}^{-}$, the phase speed can only reach $c_{s i}$ at the slightly smaller ion-neutral decoupling scale.

Slow modes exhibit a distinctive feature for wavenumbers above $k_{\mathrm{dec}, n i}$. In addition to the slow modes in ions, a new sort of slow modes emerges in neutrals, which is the "neutral slow mode" reported in Zaqarashvili et al. (2011). It is also the "neutral acoustic mode" coupled with the magnetoacoustic waves discussed in Soler et al. (2013a). It is generated below the neutral-ion decoupling scale when the slow modes on larger scales impose compression and produce perturbations in 


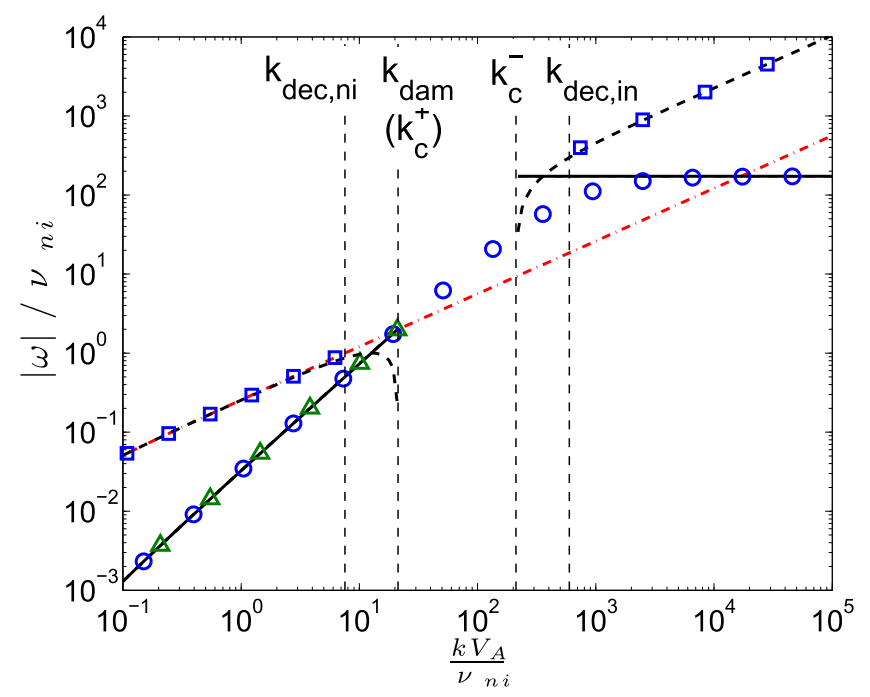

Figure 2. The same as Figure 1 but for an MC. The same symbols as in Figure 1 are used, except that the dashed line and open squares are the numerical (Equation (3)) and analytical (Equations $(5 a)$ and $(6 a))\left|\omega_{R}\right|$.

the neutral fluid. In the rest of the paper, we will term the two branches of slow modes below the scale $1 / k_{\mathrm{dec}, n i}$ as "neutral" and "ion" slow modes, respectively, and term the slow modes in the strongly coupled regime and the ion slow modes together as "usual" slow modes. Next we focus on the neutral and ion slow modes within the wavenumber range $\left[k_{\mathrm{dec}, n i}, k_{\mathrm{dec}, i n}\right]$.

If we extend the wave frequencies of slow modes in the strongly coupled regime (Equation (13)) to the scale $k_{\mathrm{dec}, n i}$, we find they reach the values

$$
\begin{aligned}
& \omega_{R}^{2}\left(k_{\mathrm{dec}, n i}\right) \approx \nu_{n i}^{2} \cos ^{2} \theta, \\
& \omega_{I}\left(k_{\mathrm{dec}, n i}\right) \approx-\frac{\nu_{n i}}{2} \sin ^{2} \theta,
\end{aligned}
$$

where we use the approximations $\xi_{n} \sim 1$ and $c_{s n} \sim c_{s}$. Starting from $k_{\mathrm{dec}, n i}$, the slow modes arising in neutrals have wave frequencies of

$$
\begin{gathered}
\omega_{R}^{2}=c_{s n}^{2} k^{2}, \\
\omega_{I}=-\frac{\nu_{n i}}{2} \sin ^{2} \theta .
\end{gathered}
$$

The propagating component $\omega_{R}$ has the expression corresponding to pure acoustic waves in neutrals. The imaginary part $\omega_{I}$ has the same value as $\omega_{I}\left(k_{\mathrm{dec}, n i}\right)$ in Equation $(57 b)$. By setting $\left|\omega_{R}\right|=\left|\omega_{I}\right|$, we can obtain its cutoff wavenumber $\nu_{n i} /\left(2 c_{s n}\right)$, which is equal to $k_{\mathrm{dec}, n i} / 2$. Below $k_{\mathrm{dec}, i n}$, ions start to separate from neutrals. $\omega_{I}$ of the neutral slow modes becomes

$$
\omega_{I}=-\frac{\nu_{n i}}{2} \text {. }
$$

The above analytical $\left|\omega_{I}\right|$ (Equations (58b) and (59)) are shown by filled circles in Figures 6 and 7. Due to the strong anisotropy at relatively small scales, $\sin \theta \sim 1$ and the difference between Equations (58b) and (59)) is indistinguishable on the plots. The filled squares in Figure 7 specifically show the analytical $\left|\omega_{R}\right|$ (Equation (58a)). Although the slow modes in the neutrals have the properties of pure acoustic waves, they are induced by the slow modes in the strongly coupled regime and are one of the solutions to the dispersion relation of the magnetoacoustic waves in a partially ionized plasma. We treat them as an additional branch of slow modes in our analysis.

The relative damping efficiency of the ion and neutral slow modes at high wave frequencies depends on the ionization fraction of the plasma. The ratio of their damping rates at $k>k_{\mathrm{dec}, \text { in }}$ is $\chi\left(=\rho_{n} / \rho_{i}\right)$. The higher degree of ionization in the $\mathrm{WNM}$ and SC results in a smaller ratio between the two damping rates compared with other phases.

Another difference between the WNM and other environments is the absence of the cutoff region in the WNM. Also, we see from Figure 6(a) that the cascading rate of slow modes is above the damping rate of ion slow modes over all the scales. Thus the usual slow modes in the case of the WNM survive ion-neutral collisional damping. For the rest conditions, the damping scales all coincide with the cutoff boundary $k_{c}^{+}$(see Table 2 for the expression).

We next turn to the ion slow modes within the interval $\left[k_{\mathrm{dec}, n i}, k_{\mathrm{dec}, i n}\right]$. In the vicinity of $k_{\mathrm{dec}, n i}$, ions stay tightly coupled with neutrals, and their motions are overwhelmed by the acoustic perturbations in neutrals. The damping rate is determined by the difference between $\nu_{n i} / 2$ and $\left|\omega_{I}\right|$ of neutral slow modes (Equation (58b)),

$$
\left|\omega_{I}\right|=\frac{\nu_{n i}}{2}-\frac{\nu_{n i}}{2} \sin ^{2} \theta=\frac{\nu_{n i}}{2} \cos ^{2} \theta .
$$

On the other hand, ions are constrained to magnetic field lines. Driven by the magnetic perturbation, the slow modes with an effective sound speed

$$
c_{s, \mathrm{eff}}=\sqrt{\xi_{i}} c_{s i}=\sqrt{2 \gamma k_{B} T / m_{r}}
$$

as the phase speed is generated in ions, where the reduced mass is $m_{r}=\rho / n_{i}$. It originates from the coupling state of ions remaining with neutrals in this range of wavenumbers. Note that $c_{s, \text { eff }}$ is different from $c_{s}=\sqrt{\gamma k_{B} T / m_{r}}$ of the slow modes in the strongly coupled regime. The reduced mass in $c_{s}$ is $m_{r}=\rho / n$, where $n=2 n_{i}+n_{n}$ is the total number density. It does not distinguish the neutral and ionized components since neutrals and ions are frozen together and move as one fluid. But in the range $\left[k_{\mathrm{dec}, n i}, k_{\mathrm{dec}, i n}\right]$, ions experience frequent collisions with neutrals and hence cannot oscillate freely. In the meantime, the occasional collisions acting on neutrals are inadequate to transfer inertia instantly to neutrals. As a result, we see from the expression of $c_{s, \text { eff }}$ that ions alone carry the total mass of the medium to move.

The ion slow modes can only become propagating when the real wave frequency $\left|\omega_{R}\right|=c_{s, \text { eff }} k \cos \theta$ becomes larger than the damping rate $\left|\omega_{I}\right|$ (Equation (60)). By applying the cutoff condition $\left|\omega_{R}\right|=\left|\omega_{I}\right|$, we obtain the cutoff scale

$$
k_{c, t 1}=\frac{\nu_{n i} \cos \theta}{2 c_{s, \mathrm{eff}}},
$$

as the lower wavenumber boundary of the ion slow modes within $\left[k_{\mathrm{dec}, n i}, k_{\mathrm{dec}, i n}\right]$. The ion slow modes emerge at $k_{c, t 1}$, but the phase speed $c_{s, \text { eff }}$ can only be reached above the wavenumber

$$
k_{\mathrm{dec}, t 1}=\frac{\nu_{n i} \cos \theta}{c_{s, \mathrm{eff}}}
$$

satisfying $\left|\omega_{R}\right|=\nu_{n i} \cos ^{2} \theta$. It actually signifies that ions no longer follow the acoustic motions in neutrals, but start to develop their own wave modes propagating along the magnetic field. 


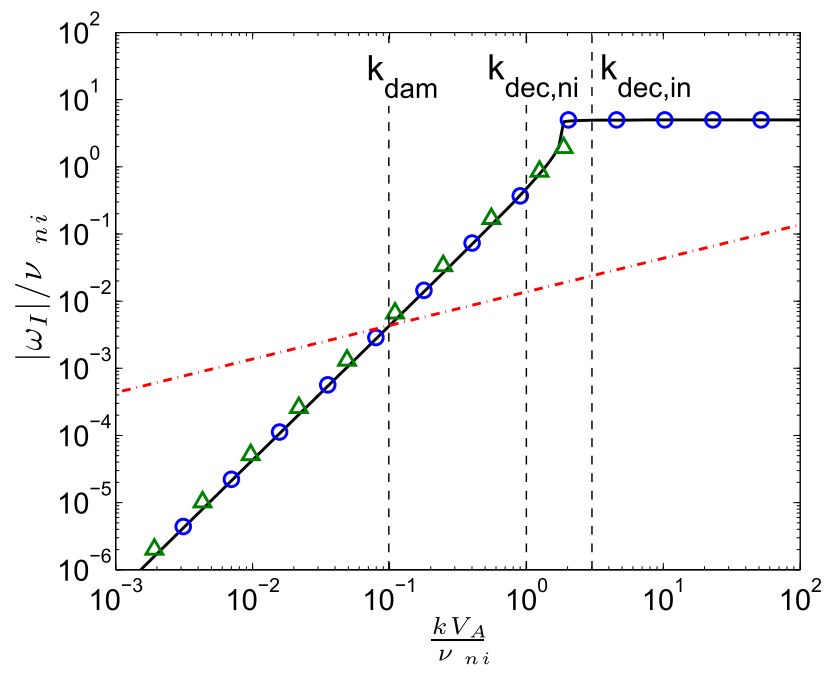

(a) WNM

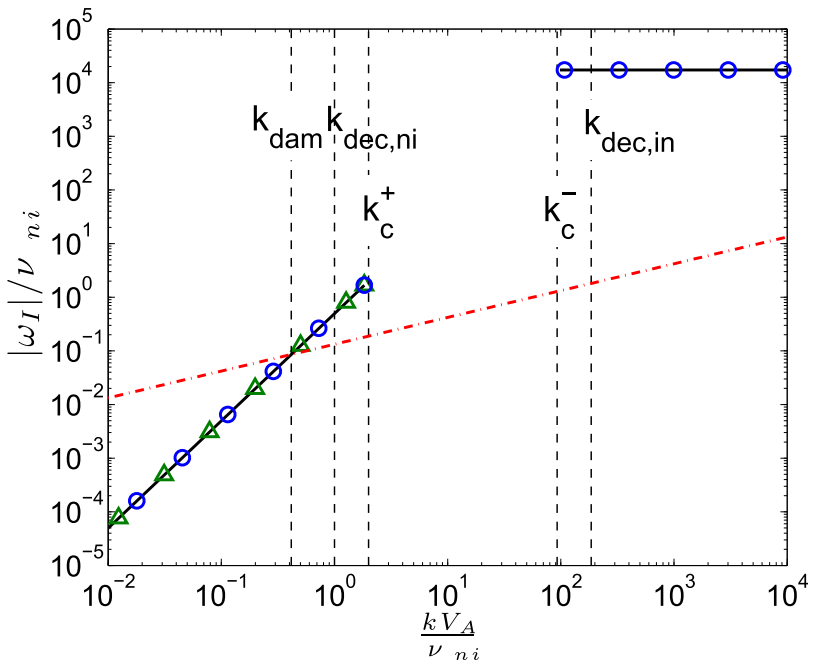

(c) DC

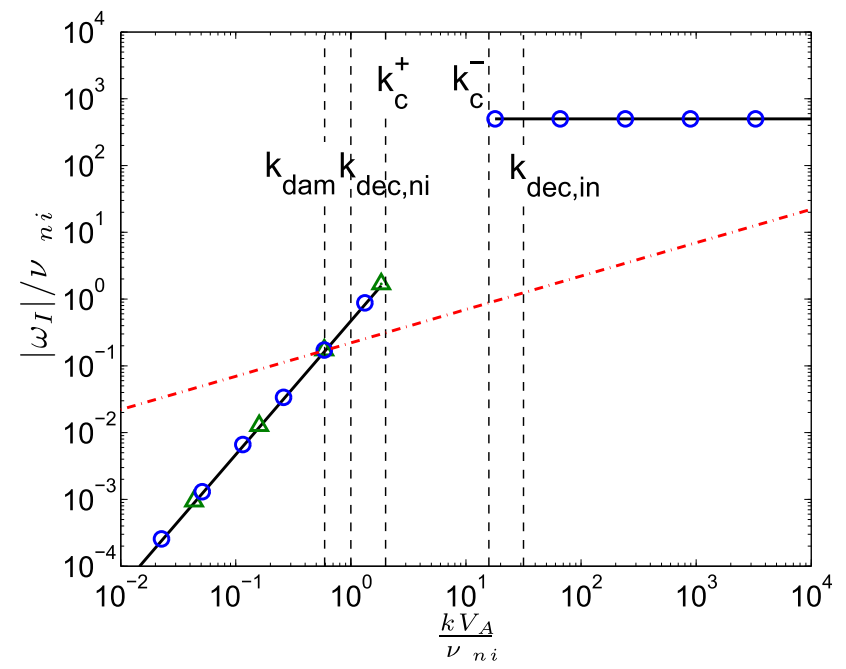

(b) $\mathrm{CNM}$

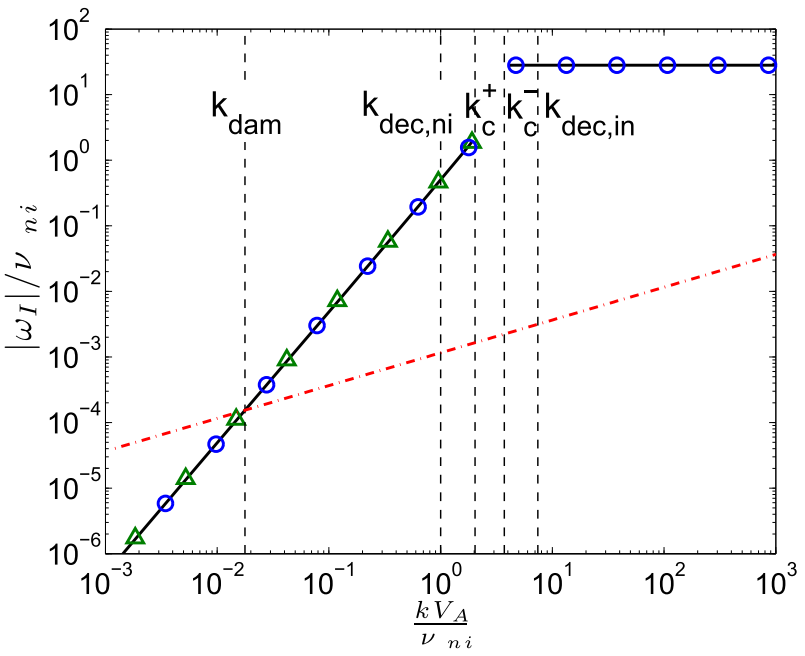

(d) $\mathrm{SC}$

Figure 3. The same as Figure 1 but for fast modes. Here we set $\theta=45^{\circ}$ as an example. Solid lines are numerical solutions to equation (51) in Soler et al. (2013a). For the analytical results, circles correspond to the two-fluid damping rate (Equations (12b) and $(6 b)$ ), and triangles are the single-fluid damping rate (Equation (27b)). Dashed-dotted lines are the cascading rates given by Equation (43).

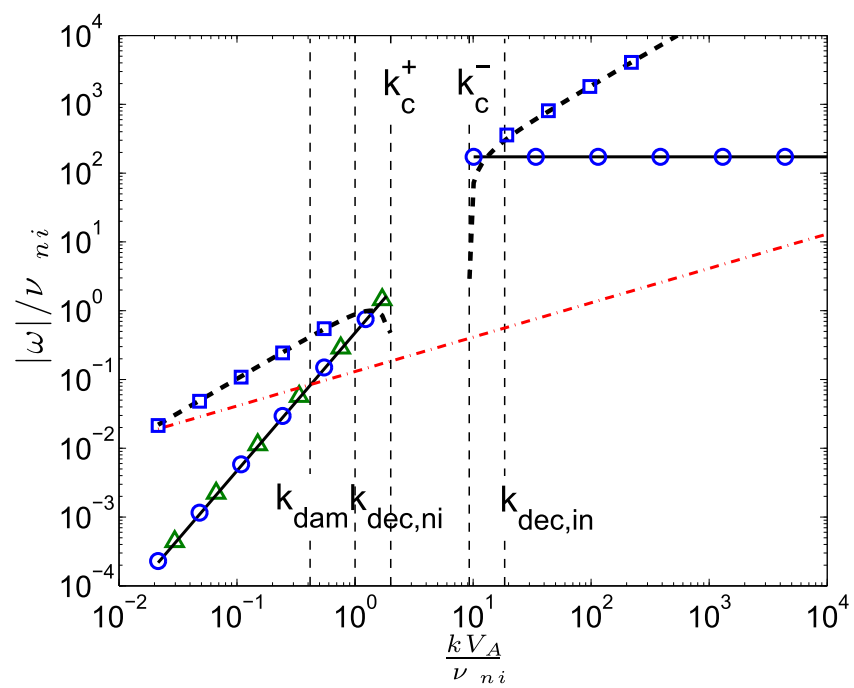

Figure 4. The same as Figure 3 but for MCs. The dashed line and open squares are the numerical (Equation (51) in Soler et al. 2013a) and analytical (Equations (12a) and (14)) $\left|\omega_{R}\right|$

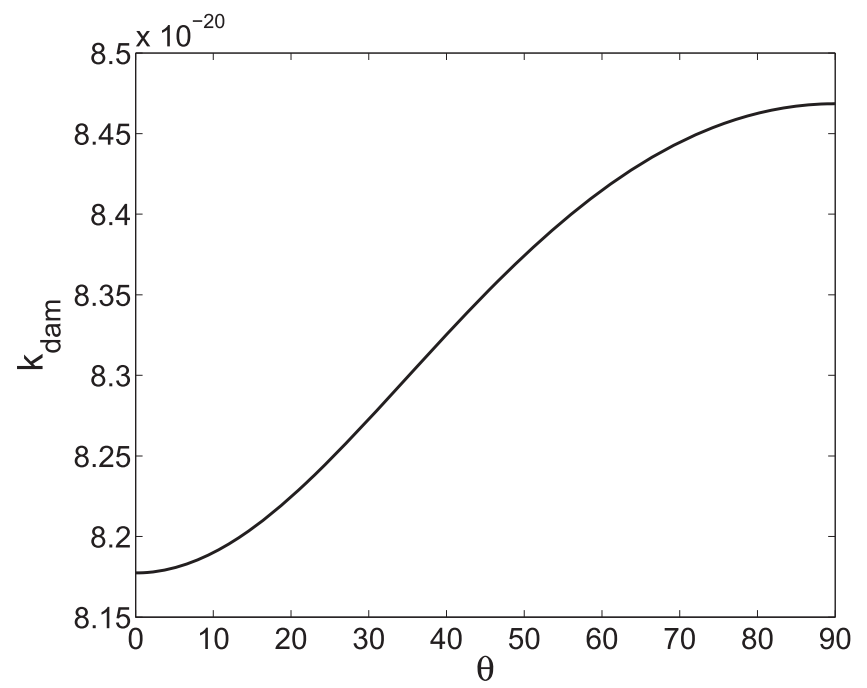

Figure 5. $k_{\mathrm{dam}}$ of fast modes as a function of $\theta$ in the WNM (Equation (46b)). 


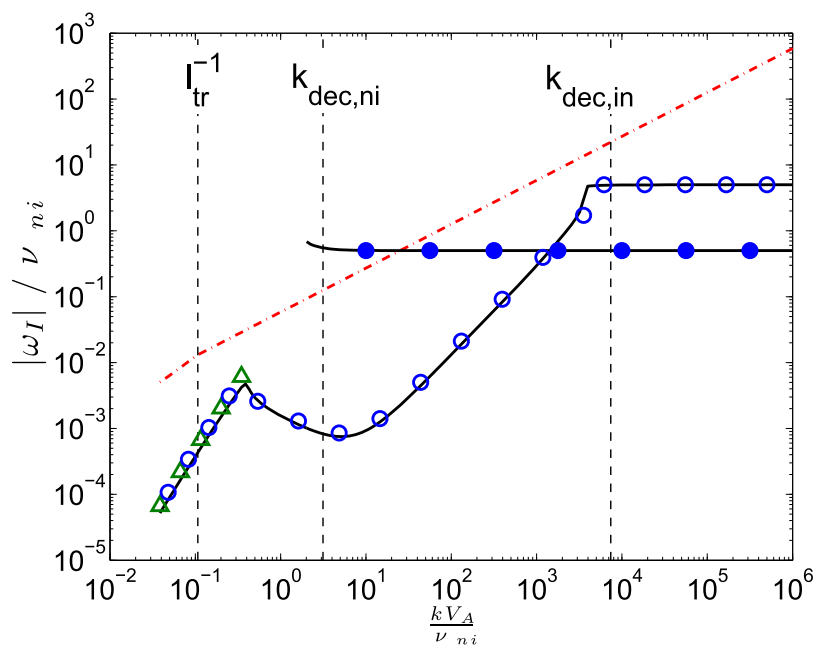

(a) WNM

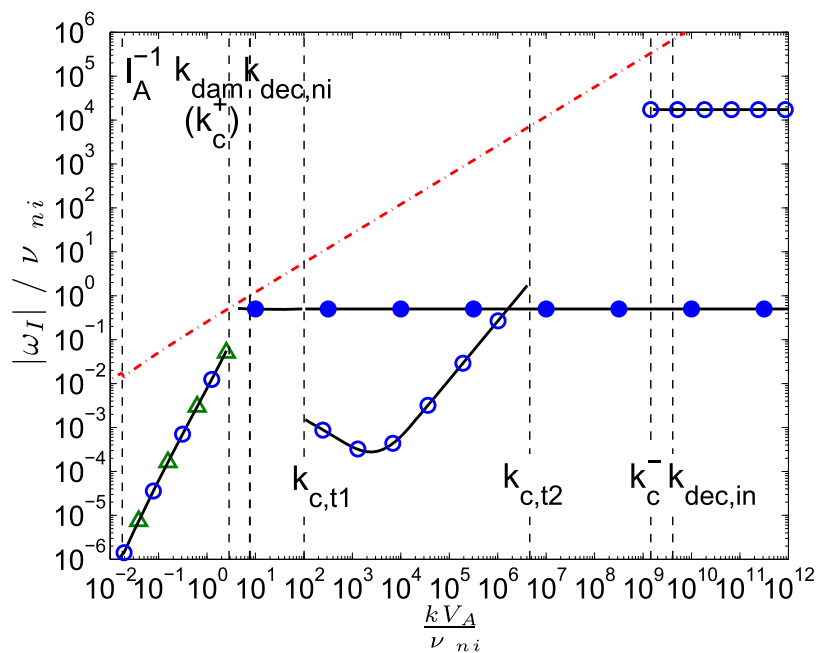

(c) DC

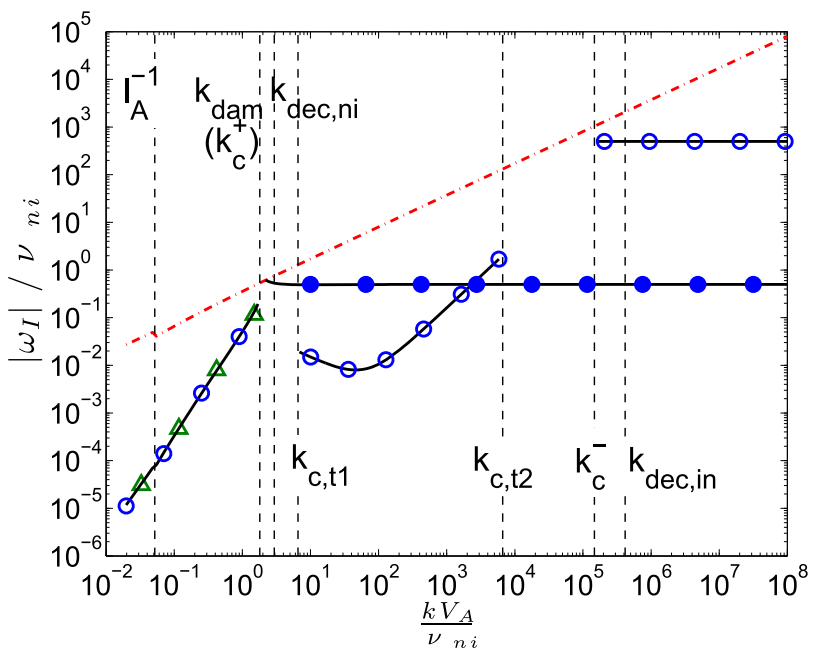

(b) $\mathrm{CNM}$

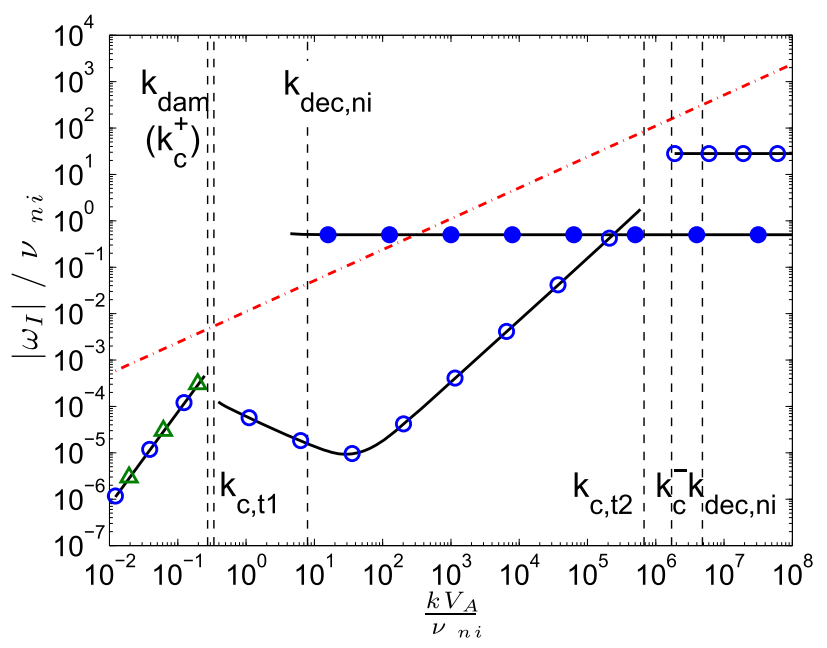

(d) $\mathrm{SC}$

Figure 6. The same as Figure 1 but for slow modes. Solid lines are numerical solutions to equation (51) in Soler et al. (2013a). Only one branch of slow modes exists in the strongly coupled regime. Circles and triangles represent the analytical damping rates from two-fluid (Equation (13b)) and single-fluid (Equation (28b)) approaches. For wavenumbers beyond $k_{\mathrm{dec}, n i}$, there are both neutral and ion slow modes. Their analytical damping rates are represented by filled (Equations $(58 b)$ and (59)) and open (Equations (65b) and (6b)) circles, respectively. The dashed-dotted lines are the same cascading rates as those of Alfvén modes (Equations (29) and (31)). Four cutoff boundaries $k_{c}^{ \pm}, k_{c, t 1}, k_{c, t 2}$ are indicated in the figures.

The appearance of the propagating ion slow modes induces a new component of the damping rate, associated with the wave motions. Since the ion slow modes are driven by magnetic perturbation and the propagation is guided by the magnetic field, no transverse compression can be produced. Therefore, different from the case for slow modes in the strongly coupled regime, there is only damping to the parallel propagation. Correspondingly, the new component of the damping rate is

$$
\left|\omega_{I}\right|=\frac{\omega_{R}^{2}}{2 \nu_{n i}}=\frac{c_{s, \text { eff }}^{2} k^{2} \cos ^{2} \theta}{2 \nu_{n i}} .
$$

We now obtain full expressions of the wave frequencies of the ion slow modes within $\left[k_{\mathrm{dec}, n i}, k_{\mathrm{dec}, i n}\right]$

$$
\begin{gathered}
\omega_{R}^{2}=c_{s, \mathrm{eff}}^{2} k^{2} \cos ^{2} \theta, \\
\omega_{I}=-\left(\frac{\nu_{n i}}{2} \cos ^{2} \theta+\frac{c_{s, \mathrm{eff}}^{2} k^{2} \cos ^{2} \theta}{2 \nu_{n i}}\right) .
\end{gathered}
$$

The relative importance of the two terms in Equation (65b) changes with $k$. By equaling the two terms of the damping rate, we obtain the transition scale

$$
k_{\text {tran }}=\frac{\nu_{n i}}{c_{s, \mathrm{eff}}} .
$$

When $k<k_{\text {tran }}$, the first term dominates the damping rate. It is independent of $\omega_{R}$ and corresponds to the decreasing $\left|\omega_{I}\right|$ with $k$ shown in Figures 6 and 7 under the condition of scaledependent anisotropy. For wavenumbers beyond $k_{\text {tran }}$, the second term becomes dominant, which is proportional to $\omega_{R}^{2}$ and increases toward larger $k$. Note that at $k_{\text {tran }},\left|\omega_{R}\right|=\nu_{n i} \cos \theta$ can be fulfilled, which coincides with the $\left|\omega_{R}\right|$ value at $k_{\mathrm{dec}, n i}$ (Equation (57a)) and is equivalent to the condition $c_{s, \text { eff }} k=\nu_{n i}$. We see that $k_{\text {tran }}$ not only represents the transition in the damping rate, but also corresponds to the critical wavelength within which the effective sound crossing time is equal to the neutral-ion collisional time. That is, below the scale $1 / k_{\text {tran }}$, the 


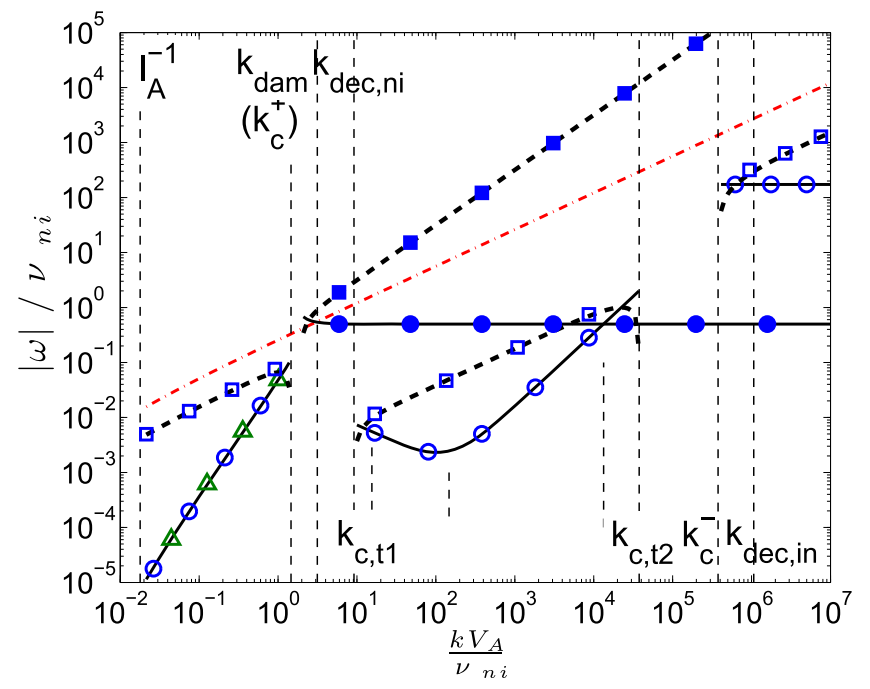

Figure 7. The same as Figure 6 but for an MC. The filled squares are from Equation $(58 a)$ for $\left|\omega_{R}\right|$ of the neutral slow modes. The open squares are from Equations (13a), (65a), and (15). The short vertical dashed lines within $\left[k_{c, t 1}, k_{c, t 2}\right]$ represent $k_{\mathrm{dec}, t 1}, k_{\mathrm{tran}}, k_{\mathrm{dec}, t 2}$ from left to right, respectively.

disturbance associated with the ion slow modes cannot be effectively transmitted to neutrals through neutral-ion collisions. Neutrals further drift apart from ions, and ions are less effectively coupled to neutrals. The differential motions between ions and neutrals become more significant, which consequently causes stronger damping to the ion slow modes.

The propagating component $\left|\omega_{R}\right|$ starts to decay when $\left|\omega_{R}\right|$ reaches the value of $\nu_{n i}$, corresponding to the scale

$$
k_{\mathrm{dec}, t 2}=\frac{\nu_{n i}}{c_{s, \mathrm{eff}} \cos \theta} \text {. }
$$

Neutrals become essentially unaffected by the ion slow modes. Subsequently $\left|\omega_{R}\right|$ and $\left|\omega_{I}\right|$ are in equality and the ion slow modes are cutoff at the scale

$$
k_{c, t 2}=\frac{2 \nu_{n i}}{c_{s, \text { eff }} \cos \theta}
$$

which is also the upper wavenumber boundary of the ion slow modes within $\left[k_{\mathrm{dec}, n i}, k_{\mathrm{dec}, \text { in }}\right]$.

The analytical $\left|\omega_{I}\right|$ (Equation (65b)) is shown by open circles within $\left[k_{\mathrm{dec}, n i}, k_{\mathrm{dec}, i n}\right]$ for all the environments in Figures 6 and 7. And $\left|\omega_{R}\right|$ given by Equation ( $\left.65 a\right)$ is also displayed by open squares in Figure 7 . The scales $k_{c, t 1}$ and $k_{c, t 2}$ are indicated by the vertical dashed lines in Figures $6(\mathrm{a})-(\mathrm{d})$. The three short vertical dashed lines within $\left[k_{c, t 1}, k_{c, t 2}\right]$ in Figure 7 indicate $k_{\mathrm{dec}, t 1}, k_{\text {tran }}, k_{\mathrm{dec}, t 2}$ from large to small scales, respectively. Their expressions using scale-dependent anisotropy are given in Appendix D.

Table 4 is a summary diagram of the critical scales and wave frequencies of ion slow modes, as well as those of the slow modes in both strongly and weakly coupled regimes. All the expressions are applicable in low- $\beta$ environments. The scale expressions presented here contain the propagation direction angle $\theta$. If we disregard the variance of $\cos \theta$ with scales, we find they are symmetrically linked by

$$
k_{\text {tran }}=\frac{k_{\mathrm{dec}, t 1}}{\cos \theta}=\frac{2 k_{c, t 1}}{\cos \theta} \approx \frac{\xi_{n} k_{c}^{+}}{2 \sqrt{\xi_{i}} \cos \theta}
$$

$$
=k_{\mathrm{dec}, t 2} \cos \theta=\frac{k_{c, t 2} \cos \theta}{2}=\frac{2 \sqrt{\xi_{i}} \cos \theta k_{c}^{-}}{\xi_{n}},
$$

where the last term in Equation (69a) is obtained by assuming $\sin ^{2} \theta \sim 1$ and $c_{s} \sim c_{s i}$. If we only focus on the relation among the critical scales smaller than $k_{\text {tran }}^{-1}$, we find

$$
2 k_{\mathrm{dec}, t 2}=k_{c, t 2}=\frac{4 \sqrt{\xi_{i}}}{\xi_{n}} k_{c}^{-}=\frac{2 \sqrt{\xi_{i}}}{\xi_{n}} k_{\mathrm{dec}, i n} .
$$

The same relation also holds for $k_{\mathrm{dec}, n i}, k_{c}^{+}, k_{c}^{-}$, and $k_{\mathrm{dec}, \text { in }}$ of the Alfvén and fast modes. That is (see expressions in Table 1 and Equations (7) and (16))

$$
2 k_{\mathrm{dec}, n i}=k_{c}^{+}=\frac{4 \sqrt{\xi_{i}}}{\xi_{n}} k_{c}^{-}=\frac{2 \sqrt{\xi_{i}}}{\xi_{n}} k_{\mathrm{dec}, \text { in }}
$$

when $\xi_{n} \sim 1$. And again we do not consider the change of $\cos \theta$ with $k$ for Alfvén waves here. In fact, the wave behavior of the ion slow modes below the scale $k_{\text {tran }}^{-1}$ fully resembles Alfvén waves over the whole range of scales we present, with $V_{A i}$ replaced by $c_{s i}$. Note that similar to $c_{s, \text { eff }}=\sqrt{\xi_{i}} c_{s i}$, there is $V_{A}=\sqrt{\xi_{i}} V_{A i}$.

The above analysis suggests that with $k_{\text {tran }}$ acting as a dividing wavenumber, the wave spectrum can be divided into two zones, i.e., $k<k_{\text {tran }}$ and $k>k_{\text {tran. }}$. The two zones have similarities in the sense that the scales $k_{c}^{+}, k_{c, t 1}, k_{\mathrm{dec}, t 1}$ in one zone play similar roles to $k_{c, t 2}, k_{c}^{-}, k_{\mathrm{dec}, \text { in }}$ in the other. But there also exist differences, resulting from the varying coupling states between neutrals and ions with scales. As already discussed, one of the main differences is that for the slow modes in the strongly coupled regime there is no damping for parallel propagation (Equation (13b)), but for the ion slow modes within $\left[k_{\text {tran }}, k_{c, t 2}\right]$, damping only appears for purely parallel propagation (Equation $(65 b)$ ). In addition, we observe that $\left|\omega_{I}\right|$ within $\left[k_{c, t 1}, k_{\text {trans }}\right]$ (Equation $\left.(65 b)\right)$ can be approximately written as

$$
\left|\omega_{I}\right| \sim \frac{\xi_{i} \cos ^{2} \theta}{\xi_{n}} \frac{\nu_{i n}}{2} .
$$

The factor $\xi_{i} \cos ^{2} \theta / \xi_{n}$ reflects much weaker frictional damping of the ion slow waves compared with $\left|\omega_{I}\right|$ (Equation $(6 b)$ ) of the slow modes in the weakly coupled regime.

To reinforce our understanding of wave behavior, we also provide the force analysis for both fast and slow waves in Appendix E.

The neutral slow modes originate from the compression produced by magnetoacoustic waves. They can only develop after neutrals decouple from ions and propagate with a sound speed in neutrals. ${ }^{8}$ In terms of turbulence properties, below the scale $1 / k_{\mathrm{dec}, n i}$, for Alfvén and fast modes in a low- $\beta$ medium, hydrodynamic turbulence starts to evolve in neutrals where no sound waves arise. But in the case of slow modes, neutrals begin to carry the acoustic turbulence caused by interacting sound waves (Zakharov 1967; Zakharov \& Sagdeev 1970; L'vov et al. 2000) at scales smaller than $1 / k_{\mathrm{dec}, n i}$. The wave motions of neutral fluids also lead to another distinctive feature of slow modes from other modes. Instead of having a purely nonpropagating interval, propagating ion slow waves appear within $\left[k_{c}^{+}, k_{c}^{-}\right]$. This suggests that in comparison with hydrodynamic motions, the wave motions of neutral slow

\footnotetext{
8 In high- $\beta$ plasma, since the fast modes will behave like sound waves, the sound waves in neutrals will become the neutral "fast" modes.
} 
Table 4

Critical Scales and Wave Frequencies of the Slow Modes

\begin{tabular}{|c|c|c|c|c|c|c|c|c|}
\hline Regimes & Strongly Coupled & & & "ns Coupled with Neutra & & & Weakly C & pled \\
\hline Scales & $k_{c}^{+}$(Equation $\left.(17 a)\right)$ & $\begin{array}{c}k_{c, t 1} \text { (Equation (62)) } \\
\frac{\nu_{n i} \cos \theta}{2 c_{s, \text { eff }}}\end{array}$ & $\begin{array}{c}k_{\mathrm{dec}, t 1}(\text { Equation (63)) } \\
\frac{\nu_{n i} \cos \theta}{c_{s, \mathrm{eff}}}\end{array}$ & 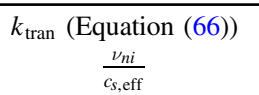 & $\begin{array}{c}k_{\mathrm{dec}, t 2} \text { (Equation (67)) } \\
\frac{\nu_{n i}}{c_{s, \text { eff }} \cos \theta}\end{array}$ & $\begin{array}{c}k_{c, t 22} \text { (Equation (68)) } \\
\frac{2 \nu_{n i}}{c_{s, \text { eff }} \cos \theta}\end{array}$ & $\begin{array}{c}k_{c}^{-}(\text {Equation }(17 b)) \\
\frac{\nu_{\text {in }}}{2 c_{s i} \cos \theta}\end{array}$ & $\begin{array}{c}k_{\mathrm{dec}, \text { in }}(\text { Table 1) } \\
\frac{\nu_{\text {in }}}{c_{s i} \cos \theta}\end{array}$ \\
\hline $\begin{array}{l}\left|\omega_{R}\right| \\
\left|\omega_{I}\right|\end{array}$ & $\begin{array}{l}<c_{s} k \cos \theta \\
\frac{\xi_{n} c_{s}^{2} k^{2} \sin ^{2} \theta}{2 \nu_{n i}}\end{array}$ & $<c_{s, \mathrm{eff}} k \cos \theta$ & & $\begin{array}{c}c_{s, \mathrm{eff}} k \cos \theta \\
\frac{\nu_{n i} \cos ^{2} \theta}{2}+\frac{c_{s, \mathrm{eff}}^{2} k^{2} \cos ^{2} \theta}{2 \nu_{n i}}\end{array}$ & & $<c_{s, \mathrm{eff}} k \cos \theta$ & $<c_{s i} k \cos \theta$ & $c_{s i} k \cos \theta$ \\
\hline
\end{tabular}


modes cause weaker friction against the magnetic pressure force on ions, and thus the ion slow modes can be driven by the persistent perturbations of the magnetic field and sustained in ions even at the "cutoff" wavenumbers.

Note that in Table 4 and the force analysis of slow waves in Appendix E, we adopt a fixed wave propagation direction and disregard its dependence on length scales for simplicity. It is necessary to point out that this treatment using a fixed propagation angle can indeed provide a simpler and more intuitively obvious description of wave behavior than that considering scale-dependent anisotropy, but it is only applicable in the analysis of linear MHD waves.

The above comparisons with numerical results show that the single-fluid approach is able to depict the damping behavior of MHD modes correctly at large scales until the cutoff boundary $k_{c}^{+}$is reached. We see for the Alfvén and slow modes, that the cutoff boundary $k_{c}^{+}$of linear waves is also the $k_{\text {dam }}$ of turbulence in the CNM, MC, DC, and SC. However, the WNM does not exhibit a cutoff region for all three wave modes. The parameter space required for the existence of wave cutoffs can be confined by equaling $k_{c}^{+}$and $k_{c}^{-}$. Due to the higher ionization degree than other ISM phases, MHD waves in the WNM are less affected by collisions with neutrals and can avoid being cut off.

Another remark needs to be made about the relation between the wave cutoff and fluid decoupling. We found that $k_{\mathrm{dec}, n i}$ $\left(k_{\mathrm{dec}, \text { in }}\right)$ and $k_{c}^{+}\left(k_{c}^{-}\right)$are of the same order of magnitude, and the interval of $\left[k_{\mathrm{dec}, n i}, k_{\mathrm{dec}, i n}\right]$ is relatively larger than $\left[k_{c}^{+}, k_{c}^{-}\right]$. Their physical connection is obvious. Only after neutrals separate themselves from the MHD wave motions of ions can they develop their own motions and exert significant influence on ions, i.e., collisional friction strong enough to cut off the MHD waves. On the other hand, although propagating waves re-emerge at $k_{c}^{-}$, they can only be fully resumed after ions are freed from coupling with neutrals at $k_{\mathrm{dec}, i n}$.

\section{APPLICATIONS TO CR PROPAGATION IN A PARTIALLY IONIZED MEDIUM}

It is necessary to introduce turbulence damping to achieve a correct understanding of the interaction of particles and MHD turbulence. Yan \& Lazarian (2002, 2004, 2008) described CR transport in a fully ionized medium and clarified that fast modes are the most effective scatterers of CRs despite their damping. It is instructive to extend their study to cover a partially ionized medium and build up a complete picture of CR propagation in the ISM. We next follow the analytical approach as presented in Yan \& Lazarian (2008) to investigate the damping effects on the scattering of CRs by MHD turbulence. Instead of using an ad hoc model of MHD turbulence, e.g., slab/two-dimensional turbulence geometry, their theory is based on the theoretically motivated (Goldreich \& Sridhar 1995; Lazarian \& Vishniac 1999; Lithwick \& Goldreich 2001) and numerically tested (Maron \& Goldreich 2001; Cho et al. 2002; Cho \& Lazarian 2002, 2003; Kowal et al. 2009, 2012; Kowal \& Lazarian 2010; Beresnyak 2014, 2015) model of MHD turbulence, and explains different sets of observational data, e.g., the "Palmer consensus" for the mean free path of CRs in the interplanetary medium (Yan \& Lazarian 2008), scattering of CRs in the warm ionized media in the Galaxy (Yan \& Lazarian 2008), particle acceleration in solar flares (Yan et al. 2008), and has also been used to describe CR physics in the super-Alfvénic turbulence in clusters of galaxies (Brunetti \& Lazarian 2007, 2011). Using the same model of MHD turbulence to study both the damping processes and $\mathrm{CR}$ propagation in a partially ionized medium ensures the selfconsistency of our results. In addition, Yan \& Lazarian (2008) demonstrated that only the turbulent eddies sampled by a CR particle have a significant scattering effect, while the magnetic perturbations on larger scales are not involved. This is consistent with the fact that the Goldreich \& Sridhar (1995) model of MHD turbulence is only valid in the reference system with respect to the local magnetic field (see the discussion in Section 4).

\subsection{Pitch-angle Scattering of CRs}

We investigate pitch-angle scattering of CRs based on the formalism of diffusion coefficients obtained from FokkerPlanck theory (Jokipii 1966; Schlickeiser \& Miller 1998; Yan \& Lazarian 2002, 2004). The energy spectra of the different modes we adopt were obtained from three-dimensional MHD simulations (Cho et al. 2002; Yan \& Lazarian 2003). In addition, we follow the nonlinear theory (NLT) employed in Yan \& Lazarian (2008) for our calculation of gyroresonance. The fluctuations of the magnetic field in MHD turbulence result in variations of particle velocities in both the perpendicular and parallel directions with regard to magnetic field lines. This substantially broadens the $\delta$-function resonance predicted in quasi-linear scattering theory, especially for large-amplitude MHD waves. In fact, the quasi-linear theory (QLT) is only applicable to infinitesimal magnetic fluctuations, which is obviously not the case for the realistic turbulent magnetic fields in diffuse ISM and MCs. The full set of expressions for the Fokker-Planck diffusion coefficient $D_{\mu \mu}$ in a low- $\beta$ medium and the notations used are presented in Appendix F (see Yan \& Lazarian 2004, 2008 for more details on the derivation).

We choose the phases of the WNM and MC as the representative examples of sub- and super-Alfvénic turbulence. Figures 8 and 9 show the calculated $D_{\mu \mu}$ (normalized by $\Omega$ ) as a function of $\mu$ for $100 \mathrm{PeV}$ CRs in the WNM and $30 \mathrm{TeV}$ CRs in the $\mathrm{MC}$, where $\mu$ is the cosine of particle pitch angle. The CRs' energy is chosen with their Larmor radius $r_{L}$ larger than the maximum damping scale among the three turbulence modes. Here

$$
r_{L}=\frac{v_{\perp}}{\Omega}, \quad \Omega=\frac{e B}{\Gamma m_{p} c} .
$$

We separately calculate the transit-time damping (TTD, solid line) for the fast and slow modes, and gyroresonance interactions (dashed line) for all turbulent modes. The gyroresonance result calculated using QLT (see Jokipii 1966; Schlickeiser 2002) is also displayed for comparison (open circles). Crosses show the approximate result using QLT for gyroresonance with fast modes, given by

$$
D_{\mu \mu}^{G}(\mathrm{QLT})=\frac{v \pi \sqrt{\mu}\left(1-\mu^{2}\right)}{4 L \sqrt{R}}\left(\frac{2}{7}-\frac{2 \sqrt{1-\mu^{2}}}{21 \mu^{2}}\right) .
$$

This simplified formula is obtained under the condition $k_{\mathrm{dam}, \perp} r_{L}<1$. Nevertheless, we see good consistency between it and the numerical integral at small pitch angles.

The diffusion coefficients in the WNM and MC exhibit similar behavior. Among the three modes, fast turbulence has the largest damping scale. Despite severe damping, the TTD from fast modes dominates CR scattering at large pitch angles. In contrast, gyroresonance is more efficient in scattering at small pitch angles. The overall contribution from Alfvén modes is smaller compared with that from fast modes. In particular in the WNM, due to the more prominent anisotropy over all scales in sub-Alfvén 


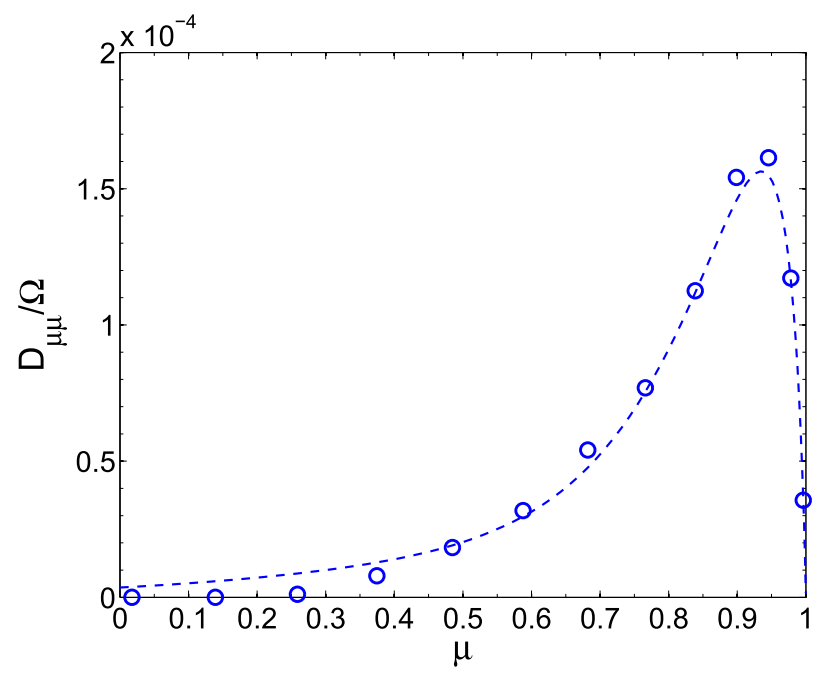

(a) Alfvén

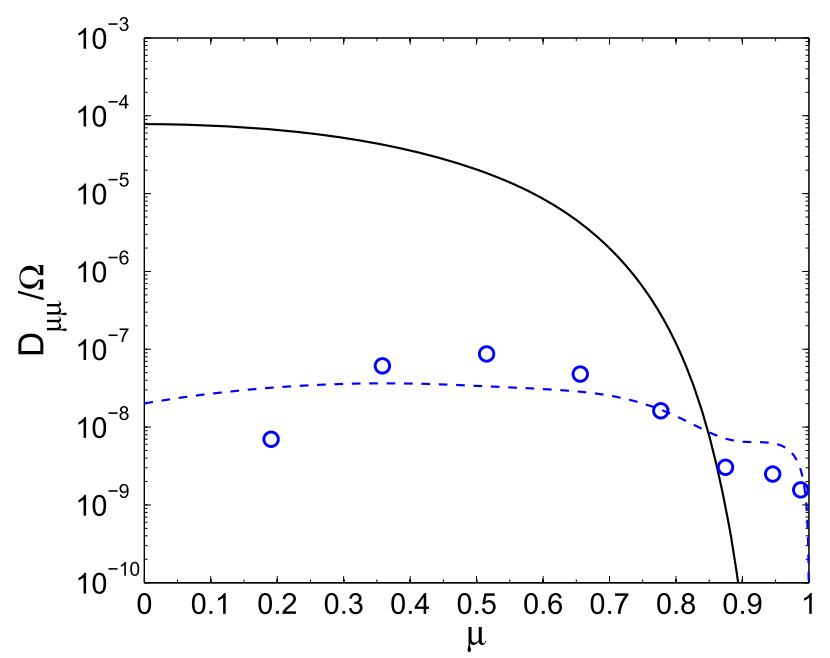

(c) Slow

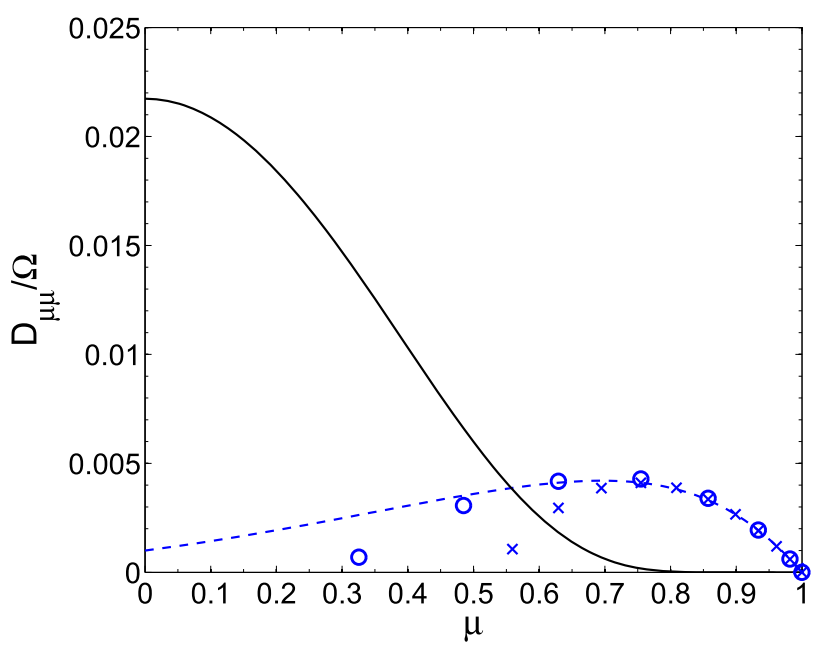

(b) Fast

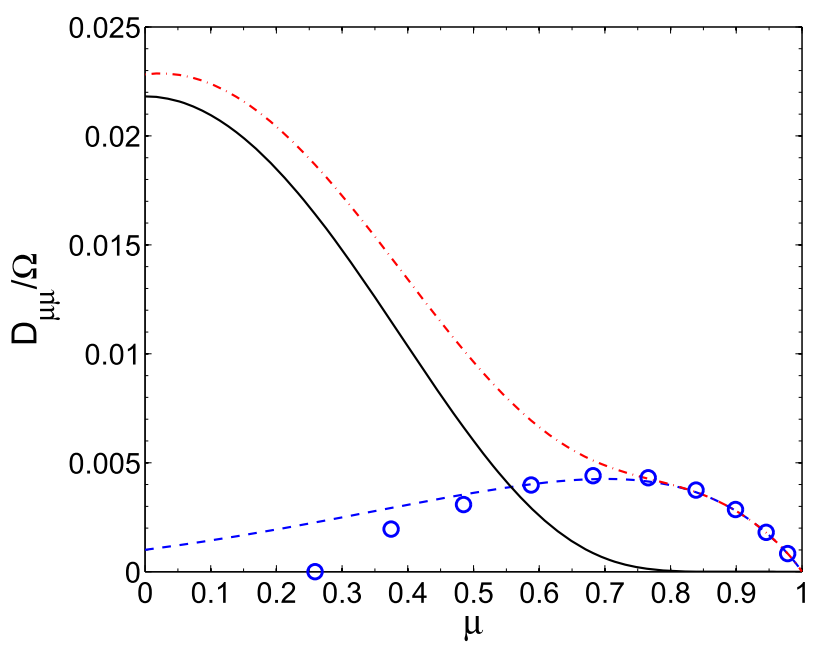

(d) Total

Figure 8. Pitch-angle diffusion coefficients (normalized by $\Omega$ ) of $100 \mathrm{PeV}$ CRs in (a) Alfvén, (b) fast, and (c) slow modes in the WNM. Solid and dashed lines refer to TTD and gyroresonance by applying NLT. Open circles are QLT results for gyroresonance. Crosses in (b) show the approximate QLT result for gyroresonance given by Equation (74). (d) Total diffusion coefficients from three modes. The dashed-dotted line is the sum of the solid and dashed lines.

turbulence, Alfvén modes are largely suppressed in CR scattering. Comparing Figures 8(b) and (d), we find that fast modes contribute alone in the total diffusion coefficient. These results are in accordance with the previous findings in Yan \& Lazarian $(2002,2004,2008)$. The scattering by slow modes depends on the $\beta$ of the medium (see Equations (133)-(135)). A low $\beta$ value in the ISM conditions (see Table 3 ) makes the effect of slow modes negligible. In addition, through comparison with the results of gyroresonance from QLT, we find that the nonlinear effect results in a much wider range of pitch angles, while QLT gives $D_{\mu \mu}=0$ at large pitch angles due to its discrete resonances, $k_{\|, \text {res }}=\Omega /(v \mu)$. This resonance condition cannot be satisfied at small $\mu$ as the turbulence is damped before cascading down to small scales. Only at the large $\mu$ end does gyroresonance from QLT come into coincidence with that from NLT.

To examine the damping effect on $\mathrm{CR}$ scattering, we again calculate the diffusion coefficients but for CRs with relatively low energies. The CRs' energies are chosen with their Larmor radius $r_{L}$ smaller than the minimum damping scale among the three turbulence modes. But since in the WNM slow modes survive ion-neutral collisional damping (see Section 5.3) and can extend to the gyroscale of ions, we choose $10 \mathrm{TeV}$ CRs with $r_{L}$ smaller than the damping scale of Alfvén modes, which is smaller than that of fast modes. For the MC, Alfvén modes have the smallest damping scale. Accordingly, we adopt $10 \mathrm{GeV}$ as the CR energy.

Figures 10 and 11 show the results in the WNM and MC respectively. The asterisks represent the simplified expression for TTD with fast modes,

$$
D_{\mu \mu}^{T}(\mathrm{NLT})=\frac{v \sqrt{\pi}\left(1-\mu^{2}\right)^{2}}{8 L \Delta \mu} \exp \left(-\frac{\mu^{2}}{(\Delta \mu)^{2}}\right)\left(\sqrt{k_{\mathrm{dam}} L}-1\right),
$$

which is obtained under the condition $k_{\mathrm{dam}, \perp} r_{L}<1$ and agrees well with integral result for low-rigidity CRs over all pitch angles. Obviously, the TTD with fast modes becomes the only significant scattering effect. The remaining gyroresonance of slow modes in the WNM has a negligible effect. Compared with the results for high-energy CRs with $r_{L}$ larger than the damping scales of turbulence modes in Figures 8 and 9, here we can clearly see the resonance gap at small pitch angles due 
The Astrophysical Journal, 826:166 (32pp), 2016 August 1

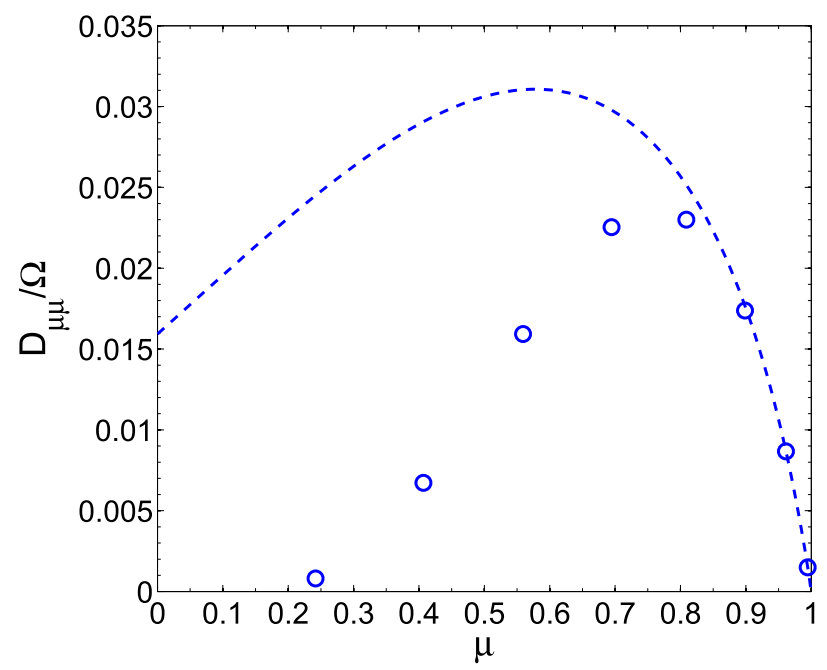

(a) Alfvén

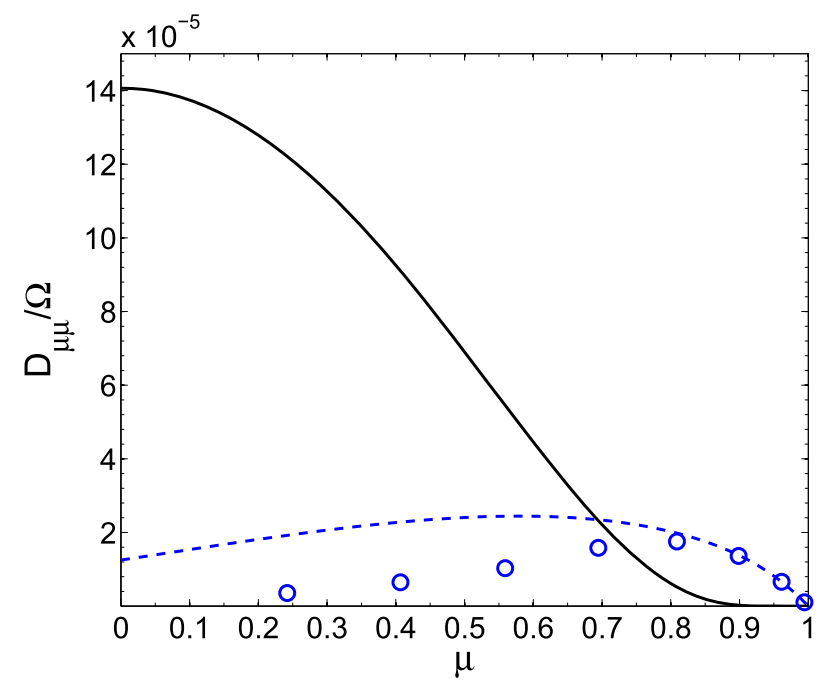

(c) Slow
XU, YAN, \& LAZARIAN

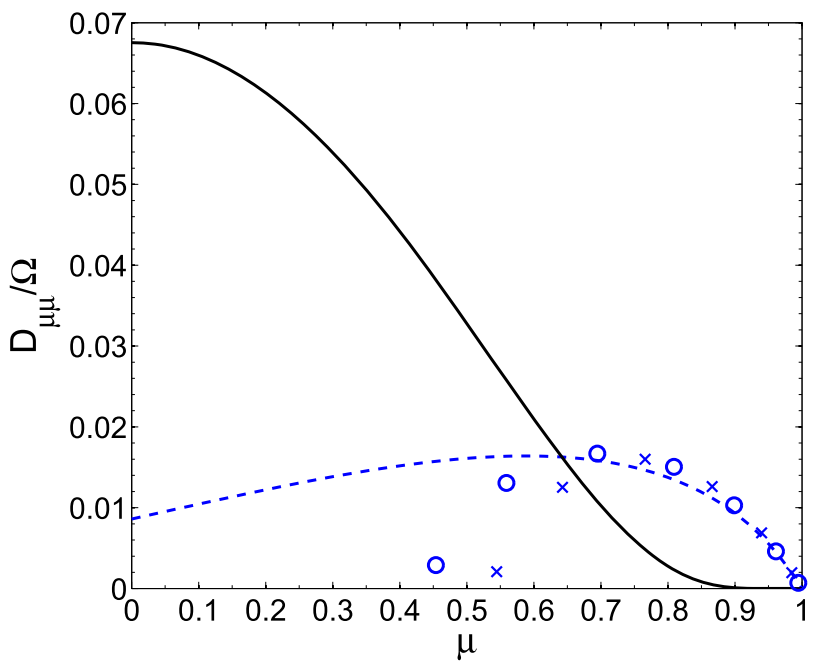

(b) Fast

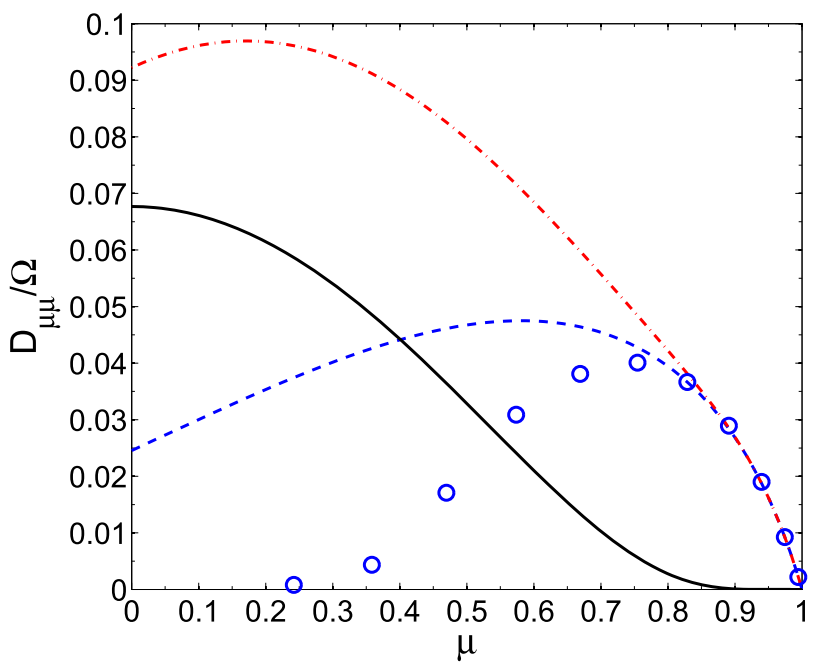

(d) Total

Figure 9. The same as Figure 8 but for $30 \mathrm{TeV}$ CRs in an MC.

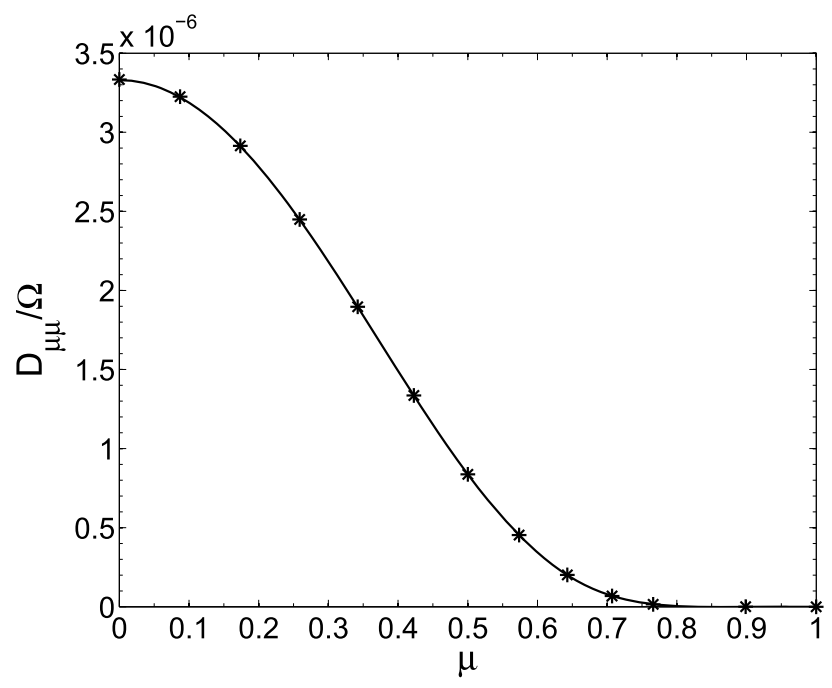

(a) Fast

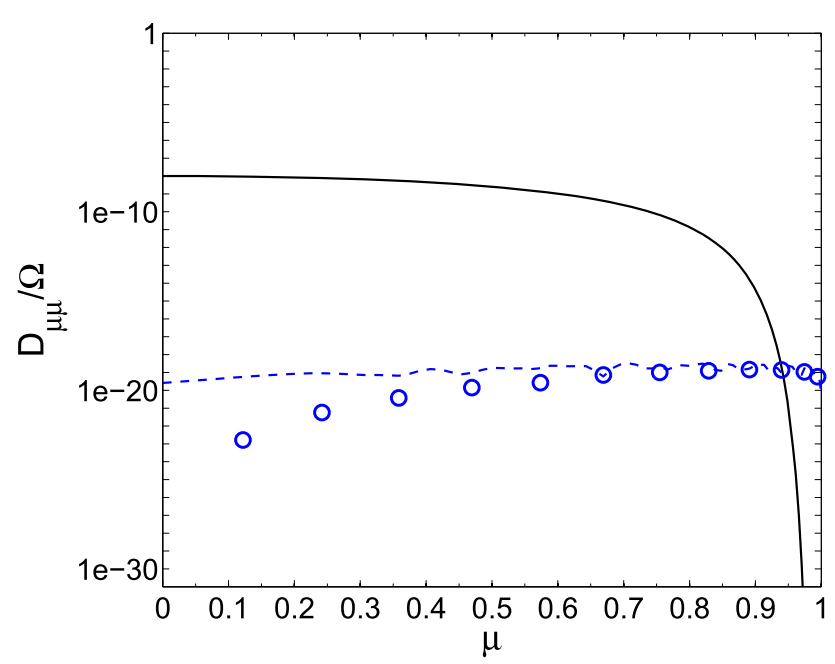

(b) Slow

Figure 10. The same as Figure 8 but for $10 \mathrm{TeV}$ CRs in (a) fast and (b) slow modes in the WNM. The asterisks in (a) are analytical results given by Equation (75). 


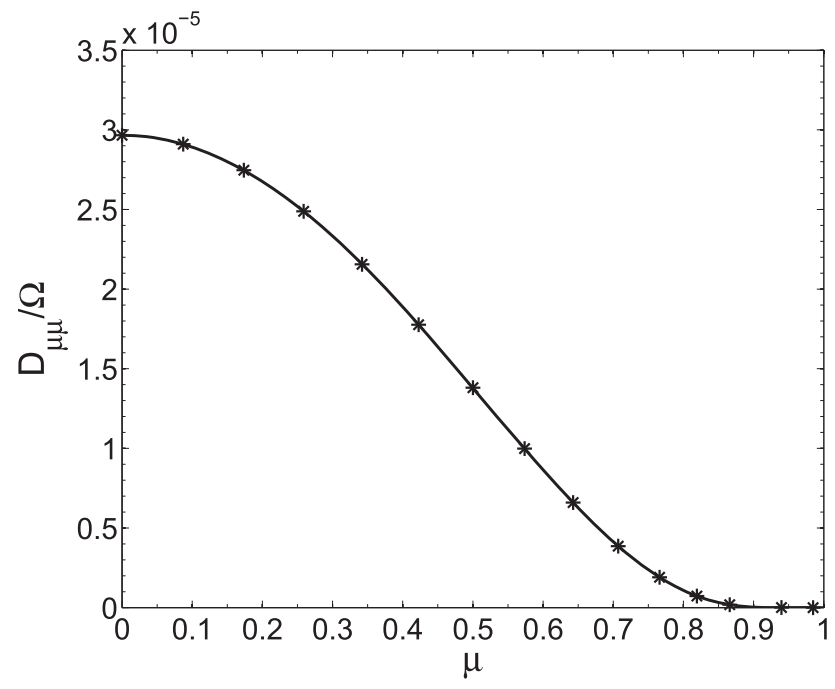

(a) Fast

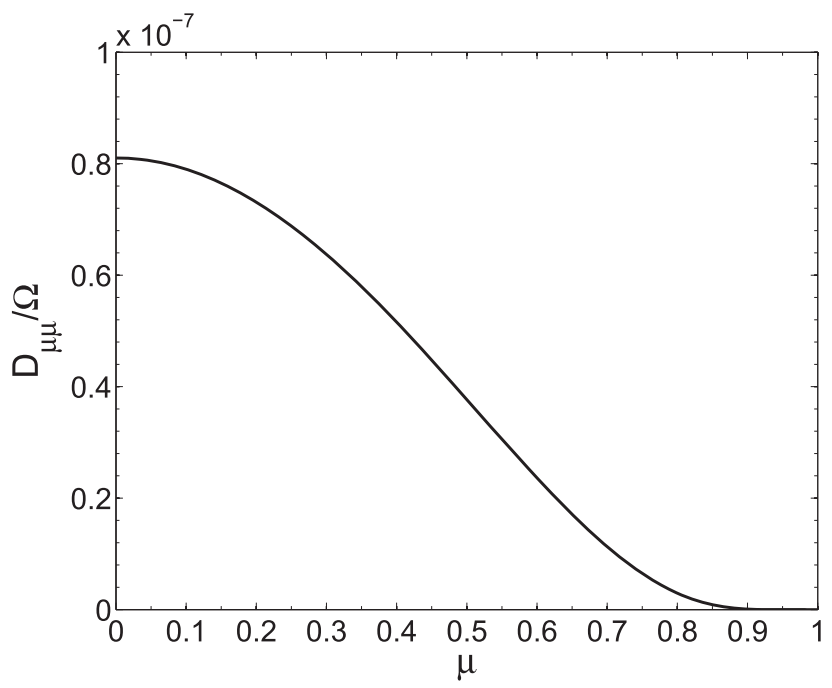

(b) Slow

Figure 11. The same as Figure 8 but for $10 \mathrm{GeV}$ CRs in (a) fast and (b) slow modes in the MC.

to the absence of gyroresonance. The TTD alone cannot fulfill the scattering of CRs with small pitch angles.

\subsection{Scattering Frequency of TTD and Gyroresonance}

To gain a deeper understanding of the dependence of TTD and gyroresonance on the CR energy and the influence of turbulence damping, we illustrate the scattering frequency $\nu=2 D_{\mu \mu} /\left(1-\mu^{2}\right)$ of the TTD and gyroresonance separately in the WNM (Figure 12) and MC (Figure 13). Since the major scattering agent differs at small and large pitch angles, we present the results of TTD at $\mu=0.1$ in Figures 12(a) and 13(a), and gyroresonance at $\mu=0.8$ in Figures 12(b) and 13(b). The vertical dashed lines indicate the $\mathrm{CR}$ energies with $r_{L}$ equal to the damping scales of Alfvén $\left(E_{\mathrm{dam}, A}\right)$ and fast $\left(E_{\mathrm{dam}, f}\right)$ modes. Slow modes are not considered due to their negligible effect.

In the WNM, as shown in Figure 12(a), when damping is absent, $\nu$ (dashed line) through TTD with fast modes decreases with CR energy. Otherwise $\nu$ (solid line) remains steady until $E_{\mathrm{dam}, f}$ (vertical dashed line) is reached, and then coincides with the result without damping. The analytical scattering frequency (asterisks) is derived by using Equation (75), and can apply to the energy range below $E_{\mathrm{dam}, f}$. As illustrated by Equation (75), for CRs with $r_{L}<k_{\text {dam }}^{-1}, D_{\mu \mu}^{T}$ depends on the damping scale. Since the CR velocity is approximately equal to the light speed and its change with energy is marginal, Equation (75) yields a constant $D_{\mu \mu}^{T}$ and $\nu$ at a fixed $\mu$. This implies that only the turbulence on scales larger than $r_{L}$ contributes to TTD interaction. As a result, in the case of no turbulence damping, $\nu$ of lower-energy CRs has a higher value because turbulence on a larger range of scales is involved in TTD scattering. While in the case with damping, $\nu$ of TTD is independent of CR energy for CRs with $r_{L}<1 / k_{\mathrm{dam}}$, and decreases with energy when $r_{L}>1 / k_{\text {dam }}$.

Figure 12(b) shows the total $\nu$ of gyroresonance from both Alfvén and fast modes in the WNM. The results free of damping and in the presence of damping come into coincidence when the CR energy is over $E_{\mathrm{dam} .}$. The respective roles of Alfvén and fast modes are explicitly displayed. When there is no damping, Alfvén modes have a negligible effect over the whole energy range due to strong turbulence anisotropy in the WNM. So $\nu$ for gyroresonance with fast modes (open triangles) coincides with the total $\nu$. The crosses are analytical $\nu$ employing Equation (74). Although Equation (74) is the simplified $D_{\mu \mu}^{G}$ using QLT for $k_{\mathrm{dam}, \perp} r_{L}<1$, the gyroresonance condition $k_{\|}=\Omega /(v \mu)=\sqrt{1-\mu^{2}} /\left(r_{L} \mu\right)$ can still be satisfied at large $\mu$, so that it can reach a good agreement with the numerical result using NLT. ${ }^{9}$ Similar to TTD, gyroresonance with fast modes has decreasing $\nu$ with increasing CR energies, but their physical origins are different. For TTD, there is not a specific resonant scale. Turbulence over all the scales above $r_{L}$ contributes to particle scattering. However, gyroresonance can only become effective on specified scales which are determined by the CR energy. We observe in Equation (74) that $D_{\mu \mu}^{G} \propto 1 / \sqrt{R}$, where $R$ is $\mathrm{CR}$ rigidity. This demonstrates that higher-energy CRs are scattered by larger-size turbulence eddies, and consequently have larger mean free paths and smaller $D_{\mu \mu}^{G}$ and $\nu$.

When damping is taken into account, since gyroresonance has a preferential resonant scale, $\nu$ corresponding to Alfvén (filled circles) and fast (filled triangles) modes only become efficient after $r_{L}$ exceeds their damping scales. The analytical $D_{\mu \mu}^{G}$ given by Equation (74) can only apply to CRs with energies larger than $E_{\mathrm{dam}, f}$ in this situation. Therefore, TTD is the only effective interaction and governs particle scattering at lower energies. Since Alfvén modes have a smaller damping scale than fast modes, their contribution can be seen within the energy range $\left[E_{\mathrm{dam}, A}, E_{\mathrm{dam}, f}\right]$, but fast modes dominate the scattering of CRs with energies higher than $E_{\mathrm{dam}, f}$.

In the case of a super-Alfvénic MC, we see much higher $\nu$ values for both TTD and gyroresonance. The $\nu$ of TTD in Figure 13(a) shows a similar trend as that in Figure 12(a) for the WNM. But for $\nu$ of gyroresonance (Figure 13(b)), the Alfvén modes become more important in the MC compared with the WNM due to less prominent turbulence anisotropy. Figures 13(c) and (d) display the components of $\nu$ from Alfvén and fast contributions for cases without and with damping. When damping is absent, $\nu$ of gyroresonance with Alfvén modes (open circles) increases toward higher energies. The opposite trend compared to gyroresonance with fast modes originates from the scale-dependent turbulence anisotropy of

\footnotetext{
9 In fact, Equation (74) is only valid for $\mu>0.5314$ to ensure a positive value of $D_{\mu \mu}$.
} 


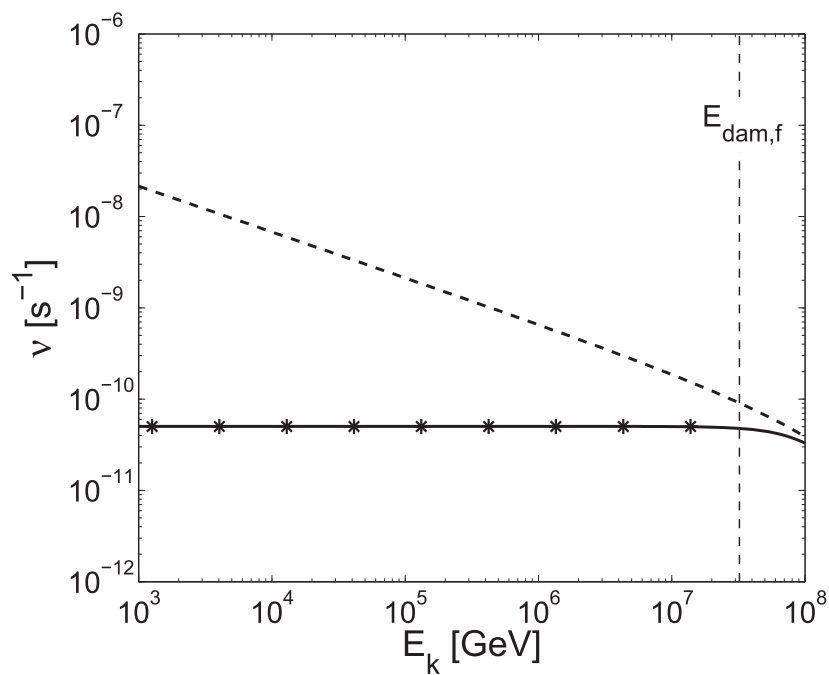

(a) TTD

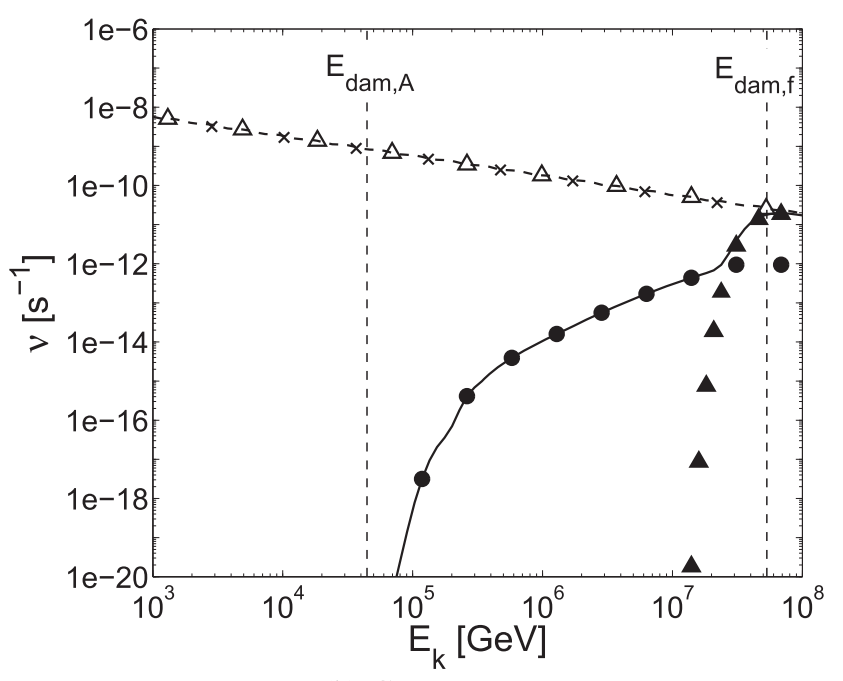

(b) Gyroresonance

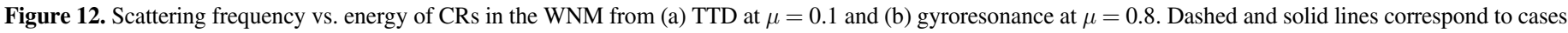

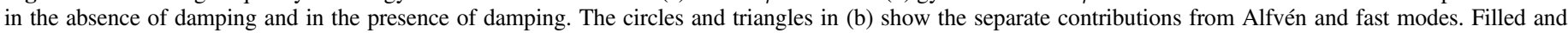

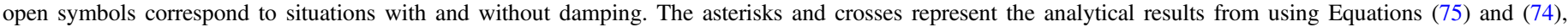
respectively.

Alfvén modes. Lower-energy CRs interact with many elongated eddies with $k_{\perp} \gg k_{\|} \sim 1 / r_{L}$ within one gyroperiod and this random walk causes inefficient scattering, while higherenergy CRs are scattered by larger-size eddies which are less anisotropic and hence the scattering efficiency increases (Yan $\&$ Lazarian 2004). We see in Figure 13(c), approaching $l_{A}$, that weak turbulence anisotropy makes Alfvén modes have a comparable efficiency to fast modes in gyroresonance scattering. Their joint effect flattens $\nu$ at high energies.

In brief, we find that the scattering frequency of both TTD and gyroresonance with fast modes decreases with the CR energy, while that of gyroresonance with Alfvén modes increases with energy. The damping effect can only make a difference in CR scattering when $r_{L}<1 / k_{\mathrm{dam}}$. Then TTD becomes the only scattering agent and has a scattering efficiency independent of particle energy. At higher energies with $r_{L}>1 / k_{\text {dam }}$, the importance of gyroresonance with Alfvén modes depends on the anisotropy degree of the turbulence. In super-Alfvénic turbulence, Alfvén modes can become more efficient than fast modes in gyroresonance scattering at large scales comparable to $l_{A}$.

\subsection{Results for the Parallel Mean Free Path of CRs}

Given the results of $D_{\mu \mu}$, the parallel mean free path $\lambda_{\|}$of CRs can be evaluated by integrating over $\mu$,

$$
\lambda_{\|}=\frac{3 v}{4} \int_{0}^{1} d \mu \frac{\left(1-\mu^{2}\right)^{2}}{D_{\mu \mu}(\mu)},
$$

where $D_{\mu \mu}$ is the total contributions from both TTD and gyroresonance with the three modes calculated using NLT. Figures 14(a)-(d) present $\lambda_{\|}$as a function of CR energy $E_{k}$. The upper limit of the energy range is restrained by $r_{L}<L$ in the WNM and $r_{L}<l_{A}$ in other environments.

We can clearly see the impact of turbulence damping on $\lambda_{\|}$. In the case of the WNM (Figure 14(a)), even in the undamped range of turbulence, neither the Alfvén nor slow modes can effectively scatter CRs. Only when the CR energy approaches $E_{\mathrm{dam}, f}$ can
CRs be confined by gyroresonance with fast modes. Nevertheless, the resulting $\lambda_{\|}$is larger than $l_{\text {tr }}$ over the whole energy range. In the WNM it shows that direct interactions with turbulence modes are invalid in scattering CRs. Instead, other mechanisms, e.g., field line wandering, may play a more important role in determining $\lambda_{\|}$.

In the other ISM phases (Figures 14(b)-(d)), we observe a nonmonotonic U-shaped dependence of $\lambda_{\|}$on the CR energy. The dramatic decrease of $\lambda_{\|}$at $E_{\mathrm{dam}, A}$ and the further decrease of $\lambda_{\|}$at $E_{\mathrm{dam}, f}$ come from the contribution of gyroresonance with Alfvén and fast modes, respectively, while the rise of $\lambda_{\|}$at $E_{k}>E_{\mathrm{dam}, f}$ is in accordance with the decreasing $\nu$ of TTD with energy. The marginal change of $\lambda_{\|}$at $E_{\mathrm{dam}, s}$ reflects the insignificant role of slow modes in CR scattering.

On the other hand, although TTD contributes over the entire energy range and is more efficient in CR scattering, it is incapable of scattering CRs with small pitch angles. Thus TTD alone is not enough to confine CRs and leads to infinite $\lambda_{\|}$in the energy range where gyroresonance is absent. Therefore, the lower-energy limit of effective CR scattering and finite $\lambda_{\|}$is determined by the damping scale of the Alfvén mode.

Table 5 summarizes the lowest energies of CRs with their scattering unaffected by turbulence damping in different partially ionized ISM phases, which are determined by the damping scales of fast modes. The scattering and acceleration of CRs with lower energies are subject to turbulence damping. Note that different to the situation discussed here, Yan \& Lazarian (2004) showed that in a fully ionized plasma, damping of fast modes strongly depends on the angle $\theta$ between $\boldsymbol{k}$ and $\boldsymbol{B}$, which results in an anisotropic distribution of fast mode energy at small scales. In their case, the lowest energy for CR scattering unaffected by turbulence damping should be a function of $\theta$.

\subsection{Other Mechanisms for Confining CRs}

Our results show that in a partially ionized medium, MHD turbulence, especially the fast modes, is severely damped by ionneutral collisions. Consequently, only high-energy CRs can be efficiently scattered through interactions with turbulence modes. In addition to scattering, the diffusion of CRs can also arise from 


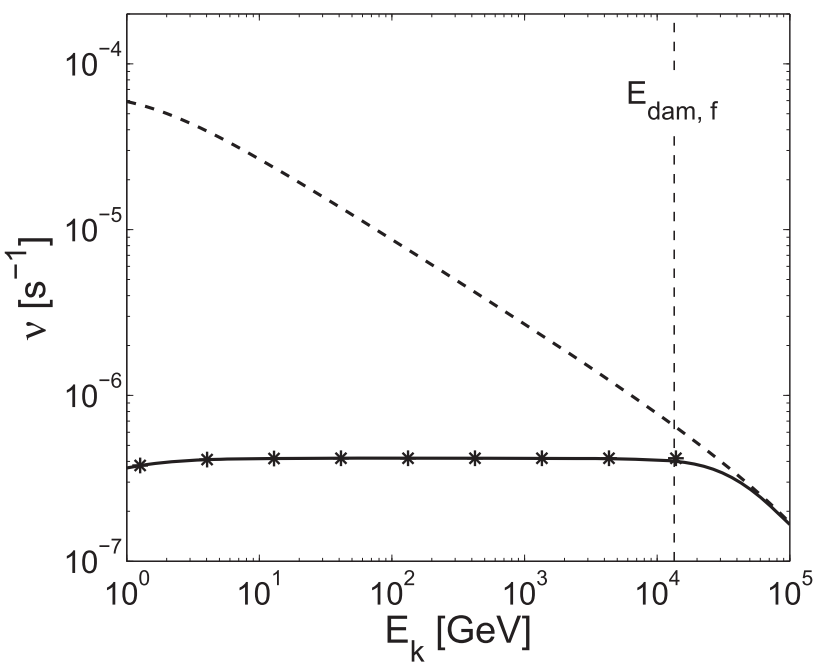

(a) TTD

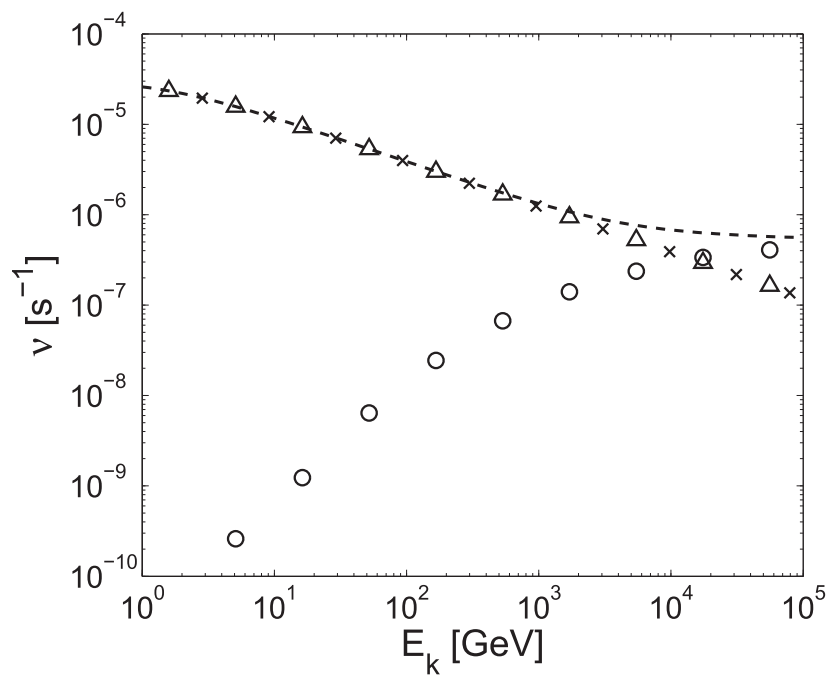

(c) Gyroresonance, without damping

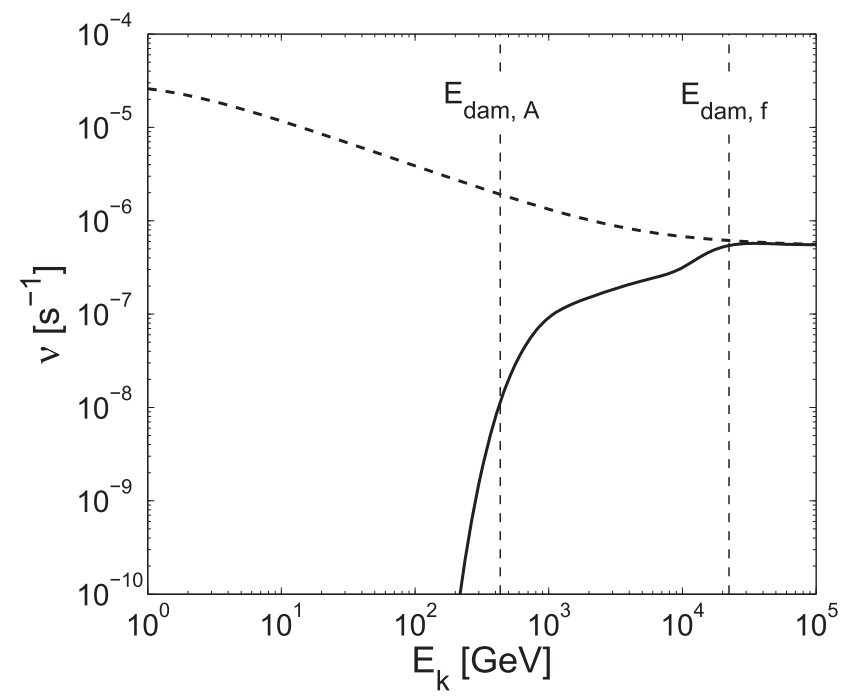

(b) Gyroresonance

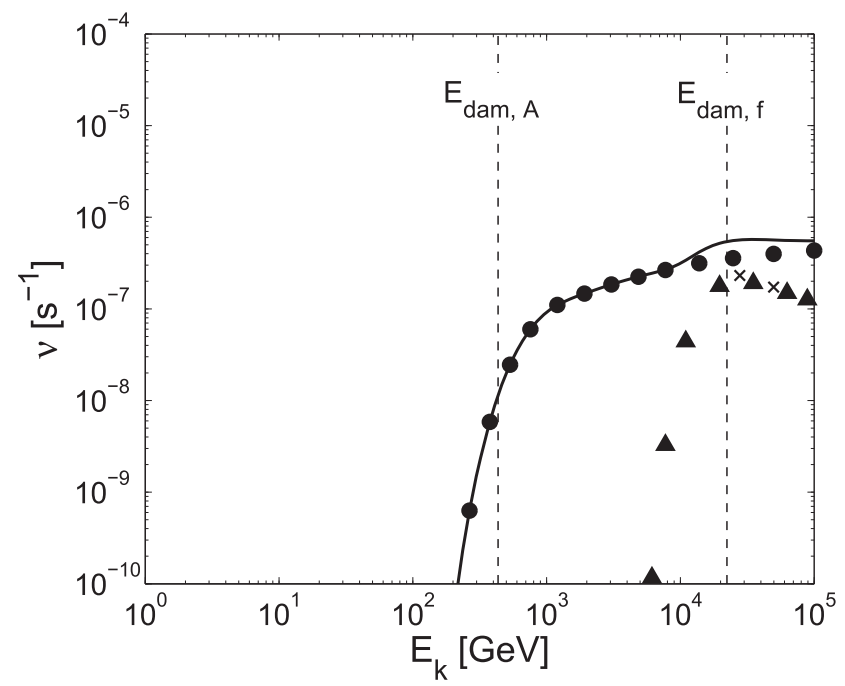

(d) Gyroresonance, with damping

Figure 13. Same as Figure 12 but for the MC. (b) is replotted in (c) and (d), showing the separate contributions from Alfvén (circles) and fast (triangles) modes.

the spatial wandering of magnetic field lines (Jokipii 1966). In three-dimensional turbulence, the wandering of field lines is induced by turbulent motions and characterized by turbulence properties. Lazarian \& Vishniac (1999) quantitively described the field line wandering according to the scaling laws of GS95-type MHD turbulence, and demonstrated its key role in determining the rate at which magnetic reconnection proceeds. Based on the Lazarian \& Vishniac (1999) prescription for magnetic field wandering and the related field line diffusion, significant theoretical reformulations of, e.g., thermal production (Narayan \& Medvedev 2001; Lazarian 2006) and CR propagation (Yan \& Lazarian 2008, 2012; Lazarian \& Yan 2014) have been achieved.

Other mechanisms which can enhance scattering of CRs include streaming instability (Wentzel 1974; Cesarsky 1980) and gyroresonance instability (Lazarian \& Beresnyak 2006; Yan \& Lazarian 2011). The additional MHD waves excited by CRs can in turn efficiently scatter CRs within a range of energies (e.g., $\lesssim 100 \mathrm{GeV}$ ). Yan et al. (2012) investigated the shock acceleration of CRs in the presence of streaming instability. They found that the CR flux near supernova remnants is strongly enhanced compared with typical Galactic values, so the growth rates of streaming instability can overcome the background turbulence damping and boost scattering of CRs. This finding enabled them to reproduce the observed gamma-ray emission from the supernova remnant W28.

The growth rate of the resonant waves excited by streaming CRs is

$$
\Gamma_{g, \mathrm{CR}} \sim \Omega_{0} \frac{n_{\mathrm{CR}}(>\Gamma)}{n_{i}}\left(\frac{v_{\text {stream }}}{V_{A}}-1\right),
$$

where $\Omega_{0}=e B / m_{p} c$ is the non-relativistic CR gyrofrequency. $n_{\mathrm{CR}}(>\Gamma)$ is the number density of CRs with energies larger than $\Gamma \mathrm{GeV}$, and the $r_{L}$ value corresponding to $\Gamma$ gives the wavevector of the generated waves, i.e., $r_{L} \sim 1 / k_{\|} \sim 1 / k$, which propagate closely parallel to the magnetic field. $v_{\text {stream }}$ is the streaming velocity of CRs, which should be larger than $V_{A}$ so as to amplify the waves.

The instability can only set in when $\Gamma_{g, \mathrm{CR}}$ exceeds the total damping rate, which includes the ion-neutral collisional and neutral-viscous damping discussed in this paper, and also the nonlinear damping by background MHD turbulence (Yan \& 


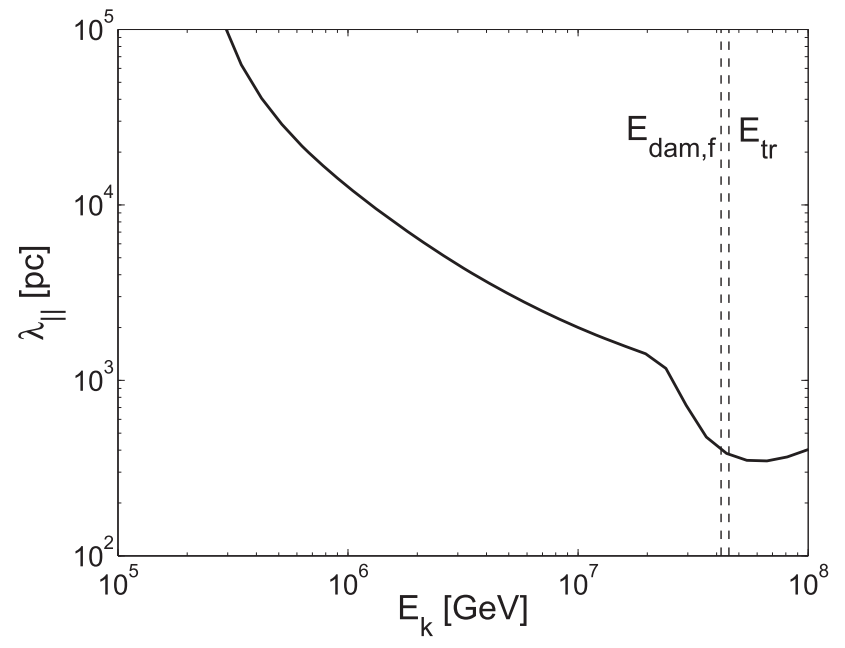

(a) WNM

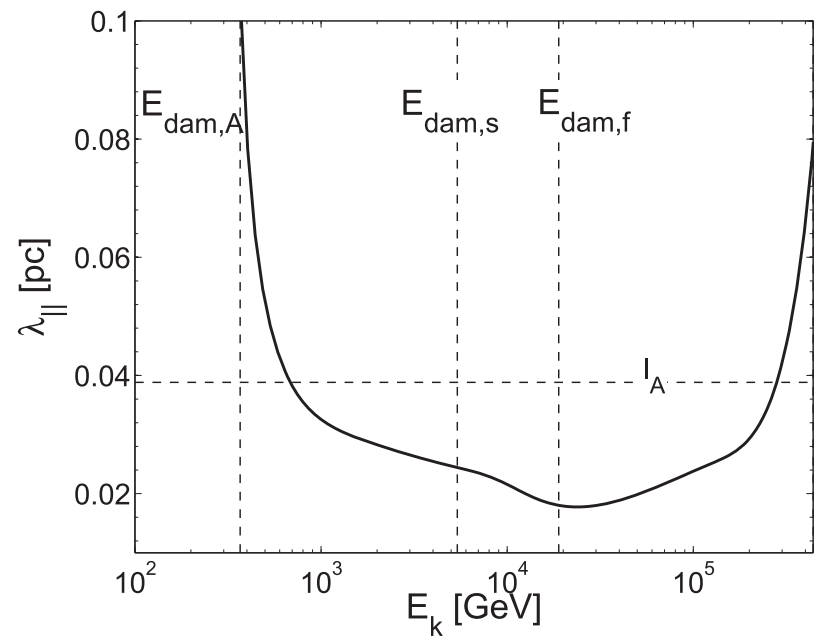

(c) $\mathrm{MC}$

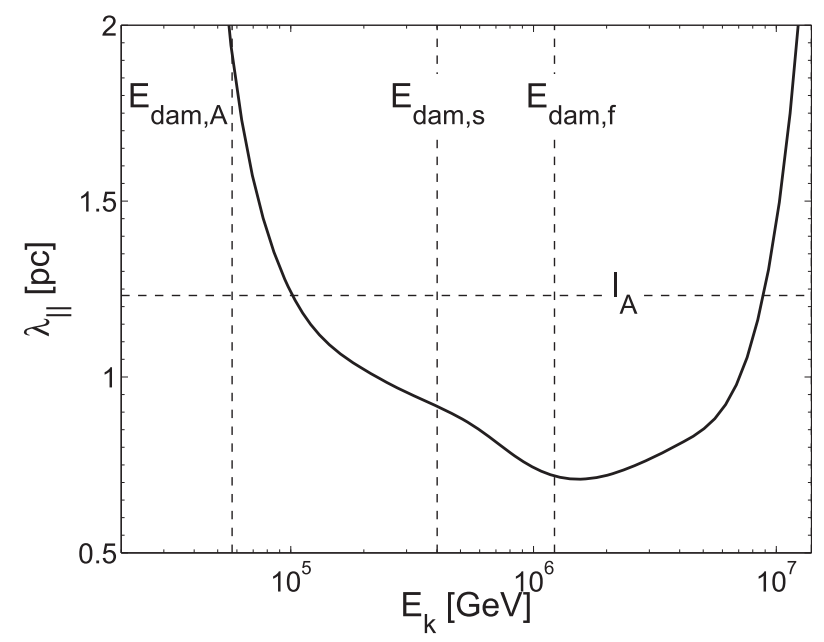

(b) $\mathrm{CNM}$

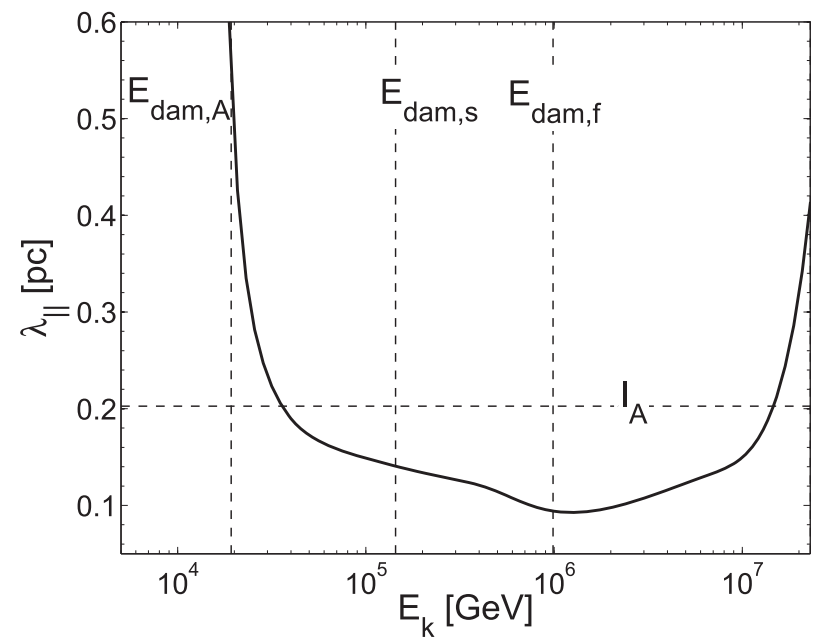

(d) DC

Figure 14. Parallel mean free path of CRs as a function of their energies in the (a) WNM, (b) CNM, (c) MC, and (d) DC. Vertical dashed lines represent the CR energies with $r_{L}$ equal to the damping scales of Alfvén $\left(E_{\mathrm{dam}, A}\right)$, slow $\left(E_{\mathrm{dam}, s}\right)$, and fast $\left(E_{\mathrm{dam}, f}\right)$ modes. Note that $E_{\mathrm{dam}, A}$ is below the energy range shown in (a). $E_{\mathrm{tr}}$ is the energy corresponding to $l_{\mathrm{tr}}$. The horizontal dashed lines in (b), (c), and (d) refer to the length scales of $l_{A}$.

Table 5

The Minimum Energy of CRs Unaffected by Turbulence Damping in Different ISM Phases

\begin{tabular}{lcccc}
\hline \hline \multirow{2}{*}{ ISM Phases } & \multicolumn{3}{c}{$k_{\text {dam }}^{-1}$} & \multirow{2}{*}{$E_{k, \text { min }}$} \\
\cline { 2 - 4 } & Alfvén & Fast & Slow & \\
\hline WNM & $0.003 \mathrm{pc}$ & $4.0 \mathrm{pc}$ & $\ldots$ & $45.3 \mathrm{PeV}$ \\
CNM & $0.005 \mathrm{pc}$ & $0.1 \mathrm{pc}$ & $0.04 \mathrm{pc}$ & $1.2 \mathrm{PeV}$ \\
MC & $6.7 \mathrm{au}$ & $0.002 \mathrm{pc}$ & $98.2 \mathrm{au}$ & $18.9 \mathrm{TeV}$ \\
DC & $35.0 \mathrm{au}$ & $0.009 \mathrm{pc}$ & $261.7 \mathrm{au}$ & $0.99 \mathrm{PeV}$ \\
\hline
\end{tabular}

Lazarian 2002, 2004, 2011; Farmer \& Goldreich 2004; Beresnyak \& Lazarian 2008). The nonlinear damping rate takes the form (Yan \& Lazarian 2011)

$$
\Gamma_{\text {turb }}=r_{L}^{-\frac{1}{2}} l_{A}^{-\frac{1}{2}} V_{A}
$$

for $r_{L}<l_{A}$ in super-Alfvénic turbulence, and

$$
\Gamma_{\text {turb }}=r_{L}^{-\frac{1}{2}} L^{-\frac{1}{2}} V_{L}
$$

for $r_{L}<l_{\text {tr }}$ in sub-Alfvénic turbulence.
As an example, Figure 15 compares the growth and damping rates at different $\mathrm{CR}$ energies in the WNM environment. The ionneutral collisional damping rate for parallel Alfvén waves (Equation (4b) with $\theta=0$ ) applies, which has the value of $\left|\omega_{I}\right|=\nu_{\text {in }} / 2$ at large wavenumbers corresponding to low CR energies. Note that neutral-viscous damping is unimportant for the generated waves in this particular case. The nonlinear damping only exists for CRs with $r_{L}$ larger than the parallel damping scale of the background Alfvénic turbulence. The parallel damping scale of Alfvénic turbulence in the case of the WNM is given by Equation (34). For the growth rate represented by the solid line, we assume $v_{\text {stream }}$ has the same order of magnitude as $V_{A}$ and adopt the CR density near the Sun, $n_{\mathrm{CR}}(>\Gamma)=2 \times 10^{-10} \Gamma^{-1.6} \mathrm{~cm}^{-3}$ (Wentzel 1974). This shows that the streaming instability can only overcome the collisional damping and effectively contribute to particle scattering for CRs with energies lower than $\sim 10 \mathrm{GeV}$. Crosses show a higher growth rate with the CR density increased by three orders of magnitude. As a result, CRs with up to $\sim 1 \mathrm{TeV}$ can be significantly scattered and confined.

From the above example we can see that to establish a comprehensive picture of $\mathrm{CR}$ propagation, other scattering mechanisms should also be incorporated in addition to direct 


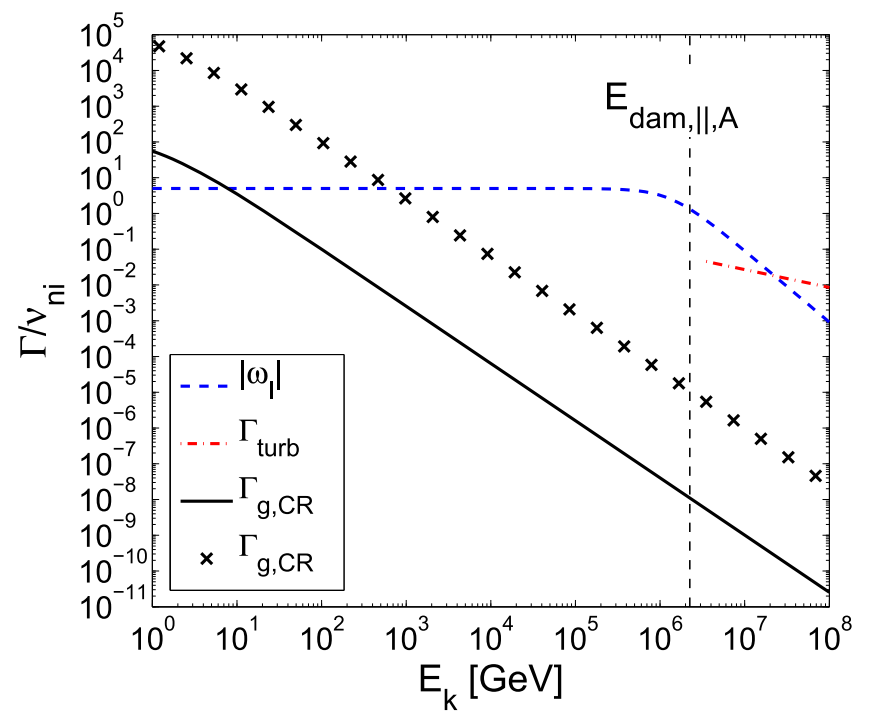

Figure 15. Growth and damping rates (normalized by $\nu_{n i}$ ) of streaming instability as a function of CR energy in WNM. The dashed line is the ion-neutral collisional damping rate. The dashed-dotted line is the nonlinear damping rate. The growth rates with different $\mathrm{CR}$ number densities, $n_{\mathrm{CR}}(>\Gamma)=2 \times 10^{-10} \Gamma^{-1.6} \mathrm{~cm}^{-3}$ (solid line), and $n_{\mathrm{CR}}(>\Gamma)=2 \times 10^{-7} \Gamma^{-1.6} \mathrm{~cm}^{-3}$ (crosses) are shown. The vertical dashed line indicates the $\mathrm{CR}$ energy with $r_{L}$ equal to the parallel damping scale of the background Alfvén modes.

interactions with turbulence modes. For relatively low-energy CRs, streaming instability can be a promising mechanism of CR confinement by scattering. For high-energy CRs with their $r_{L}$ exceeding the damping scale of MHD turbulence, TTD and gyroresonance scattering operate. For CRs with intermediate energies, their confinement is mainly attributed to field line wandering as mentioned earlier.

We again stress that in analyzing these scattering processes in a partially ionized medium, ion-neutral collisional damping is an essential physical ingredient, which determines the dissipation scale of the turbulence cascade and is the major constraint for the growth of instabilities. Taking the damping effect into account is necessary to attain realistic calculations of diffusion coefficients and a proper understanding of $\mathrm{CR}$ propagation in a partially ionized medium.

\section{DISCUSSION}

The two-fluid dispersion relations for magnetoacoustic waves used in this paper are taken from earlier works by Soler et al. (2013a) and Zaqarashvili et al. (2011), where the basic equations and detailed derivations are provided. Also, an approximate study of propagating waves (i.e., $\omega_{R}$ ) in both high and low- $\beta$ plasmas was shown in Soler et al. (2013a). However, these works mainly focus on numerical studies, which depend on the specific parameter space adopted. In the current work, we performed a comprehensive analysis of both the propagation and damping of MHD waves in a partially ionized medium, and for the first time achieved the expressions of wave frequencies for all branches of slow waves over the whole range of the wave spectrum. In addition, we present the solutions to both two-fluid and single-fluid dispersion relations derived from different analytical procedures. As the most noticeable difference, this work is devoted to the damping of MHD turbulence, thus accordingly we introduce the effect of turbulence anisotropy in the behavior of wave modes, instead of using a constant propagation angle as in Soler et al. (2013a) and Zaqarashvili et al. (2011). We furthermore reveal the physical connections between MHD turbulence and linear waves in terms of damping.

In view of the astrophysical environments dealt with in this paper, we restrict ourselves to analysis in low- $\beta$ conditions, but the simplified wave frequencies in high- $\beta$ conditions can also be easily obtained from the general solution in Equation (11). For instance, in a high- $\beta$ and weakly ionized gas, we find that the wave frequencies in the strongly coupled regime are

$$
\begin{gathered}
\omega_{R}^{2}=c_{s}^{2} k^{2}, \\
\omega_{I}=-\frac{\xi_{n} V_{A}^{4} k_{\perp}^{2}}{2 \nu_{n i} c_{s}^{2}}=-\left(\frac{2}{\beta}\right)^{2} \frac{\xi_{n} c_{s}^{2} k_{\perp}^{2}}{2 \nu_{n i}},
\end{gathered}
$$

for fast modes, and

$$
\begin{gathered}
\omega_{R}^{2}=V_{A}^{2} k^{2} \cos ^{2} \theta, \\
\omega_{I}=-\frac{\xi_{n} V_{A}^{2} k^{2}}{2 \nu_{n i}},
\end{gathered}
$$

for slow modes. Different from the low- $\beta$ case, the propagation of slow waves in a high- $\beta$ medium becomes similar to that of pseudo-Alfvén waves. But unlike the damping rate of Alfvén waves (Equation $(5 b)$ ), slow waves are subject to frictional damping in both the parallel and perpendicular directions to the magnetic field. Fast waves propagate isotropically at the speed of sound. Similar to the slow waves in a low- $\beta$ medium, the magnetic field is unaffected by purely parallel propagation of sound waves and thus damping is absent. In addition, the damping rate of the fast waves at high- $\beta$ is reduced by $\sim \beta^{-2}$ (Equation $(80 b)$ ). It reflects that sound waves are only marginally damped in an environment where thermal energy dominates over magnetic energy. In the weakly coupled regime, fast and slow waves have propagating parts of $\omega_{R}^{2}=c_{s i}^{2} k^{2}$ and $\omega_{R}^{2}=V_{A i}^{2} k^{2} \cos ^{2} \theta$, respectively, and the same damping rate as in Equation (6b). The analytical solutions under high- $\beta$ conditions can also be obtained via the single-fluid approach as described in Section 3.2, but only for the strongly coupled regime. For example, the wave frequencies at the incompressible limit are given in Equation (88) in Appendix B, which are consistent with Equations (80) and (81) at $\beta \rightarrow \infty$. Specific cases of parallel and perpendicular propagation at high- $\beta$ are also displayed in Appendix B (Equations (97) and (98)).

In a partially ionized medium, the wave behavior strongly depends on the coupling state between neutrals and ions. We specifically distinguish the neutral-ion and ion-neutral decoupling scales of three modes and the regimes with different coupling degrees separated by them. The cutoff boundaries of waves are closely correlated with the decoupling scales. In fact, the cutoff interval approximately shares the same domain as that between the neutral-ion and ion-neutral decoupling scales. This coincidence indicates that fluid decoupling can be the physical origin of the wave cutoff. Unlike Alfvén and fast waves, propagating waves can arise within the original cutoff region of slow waves, which results in two cutoff intervals sitting among three propagating wave branches. We provide full analytical expressions of all the cutoff boundaries and the wave frequencies of both neutral and ion slow waves in different coupling regimes. Slow waves on scales within $\left[k_{\mathrm{dec}, n i}, k_{\mathrm{dec}, i n}\right]$ exhibit more complicated properties in both the propagating and damping 
components of the wave frequencies. Accordingly, the wave spectrum on intermediate scales is also divided into different coupling regimes, confined by multiple critical scales which are linked together in a symmetric pattern (see Section 5.3). The numerical evidence for the existence of ion slow modes can be found in, e.g., Oishi \& Mac Low (2006); Li et al. (2008). By performing two-fluid MHD simulations, they confirmed the propagation of slow MHD waves below a scale of $\sim V_{A} / \nu_{n i}$. In addition, the neutral slow modes were also identified in numerical simulations by Tilley \& Balsara (2008). We caution that depending on the ionization fraction and wave propagation direction, the disparity between $k_{c, t 1}$ (Eq. (62)) and $k_{\mathrm{dec}, n i}$ (Table 1) can be large. Accordingly, there can be an extended range of wavenumbers where only one branch of slow modes exists, and the numerical simulations with insufficient resolution cannot capture both ion and neutral slow modes at small scales. Moreover, the numerical setup for the wave propagation direction also matters since the ion slow modes are more severely damped at a smaller $\theta$ (see Table 4 and Fig. 16(c)).

In order to test the validity of the single-fluid approach, we adopted Newton's iteration method to solve the single-fluid dispersion relation. By comparing the solutions with those for the two-fluid dispersion relation under the weak damping assumption, we see consistent results at large scales down to the lower cutoff boundary $k_{c}^{+}$. Different to the previous findings in Zaqarashvili et al. $(2011,2012)$, we found that for both the cutoff scale $\left(k_{c}^{+}\right)$of Alfvén waves and the damping rate of slow waves, the results from single-fluid and two-fluid approaches are in good agreement. We further numerically confirmed the validity of the single-fluid approximation in describing MHD waves in partially ionized ISM and SC environments. This means that, for practical purposes, the single-fluid treatment can also be used to determine the damping scales of both incompressible and compressible turbulence modes in the strongly coupled regime under similar conditions.

To obtain the damping scales of MHD turbulence, we follow the present-day understanding of turbulence and treat the turbulence cascades of the Alfvén, fast, and slow modes separately. It turns out that the scale-dependent anisotropy of turbulence plays a critical role in regulating wave behavior and deriving turbulence damping. We see a close relation between the cutoffs appearing in linear MHD waves and the damping of MHD turbulence. In particular, because of the intrinsic critical balance of the turbulent motions (GS95), the cascade of Alfvén modes is truncated at the lower cutoff boundary where the nonpropagating waves arise. As a result, the wave cutoff should also be taken into account when deriving turbulence damping scales.

In addition, we apply the analytical results on turbulence damping to a variety of partially ionized ISM phases and the SC.

1. We find that neutral viscosity plays a significant role in damping Alfién modes in the WNM and SC, while ionneutral collisions act as the dominant damping effect in the other environments.

2. Fast modes under all conditions are damped out in the strongly coupled regime. The damping is particularly severe in the WNM and SC because the cascading rates in both environments are substantially low and, in addition, the damping rate in the WNM is relatively high due to its low density. In addition, different from the case of a fully ionized medium, the damping of fast modes only has a weak dependence on the wave propagation direction.
3. In the case of slow modes, since the cutoff appears earlier than turbulence damping, the damping scales of the slow modes are given by the largest cutoff scales under all conditions except for the WNM. Due to the absence of cutoff intervals, slow modes in the WNM survive ionneutral collisional damping. In fact, all three modes in the WNM do not have cutoffs. The physical explanation for this is that, due to the relatively high ionization fraction in the WNM, coupling of neutrals with ions on larger scales is easier since every neutral has more opportunities to collide with ions, and decoupling of ions from neutrals on smaller scales is also easier since every ion has fewer chances to collide with neutrals. Consequently, the separation between the neutral-ion and ion-neutral decoupling scales is much shorter and cutoff can be avoided.

As one important application of the present study, we explored the effect of damping on CR propagation in a partially ionized medium. The local reference system where GS95-type scalings stand is very important for studying CR propagation, since the scattering of a CR particle is determined by its interaction with the local magnetic field perturbation instead of the background field. This ensures realistic statistics of magnetic fluctuations which we use to calculate CR scattering.

Alfvén modes are found to be ineffective scatterers of CRs (Chandran 2000; Yan \& Lazarian 2002, 2004). During the energy cascade of Alfvén modes, turbulent eddies become more and more elongated along magnetic field lines, with energy concentrating in the direction perpendicular to the local mean magnetic field. Thus a CR particle with $r_{L}$ comparable to the parallel scale of eddies interacts with many uncorrelated eddies in the perpendicular direction within one gyroperiod. The random walk leads to very inefficient gyroresonance scattering of CRs by Alfvén modes. But in the presence of ion-neutral collisional damping, the turbulence cascade is heavily damped and truncated at a large scale, where turbulence anisotropy is relatively weak. In particular in super-Alfvénic turbulence, turbulent eddies are nearly isotropic when $r_{L}$ approaches $l_{A}$. As a result, high-energy CRs can be effectively scattered by gyroresonance with Alfvén modes.

Other effects on CR scattering, e.g., the nonlinearity of strong Alfvénic perturbations and turbulent reconnection (Brunetti \& Lazarian 2016), are not included in the current study. Their potential importance will be explored in a future work.

\section{SUMMARY}

In this paper we continued the work in Paper I on damping of MHD turbulence in a partially ionized medium. We place more emphasis on the compressible modes and apply the analytical results to a variety of low- $\beta$ ISM phases and the SC. As an important application, we studied CR propagation in a partially ionized medium considering the ion-neutral collisional damping, as well as neutral-viscous damping, of MHD turbulence. Here we summarize the main results.

1. We present an explicit correlation between the cutoff boundaries of MHD waves and decoupling scales. Under the consideration of scale-dependent anisotropy, they are

$$
k_{c}^{+}=\left(\frac{2}{\xi_{n}}\right)^{\frac{3}{2}} k_{\mathrm{dec}, n i}
$$




$$
k_{c}^{-}=2^{-\frac{3}{2}} k_{\mathrm{dec}, \text { in }}
$$

for Alfvén modes, and

$$
\begin{gathered}
k_{c}^{+}=\frac{2}{\xi_{n}} k_{\mathrm{dec}, n i}, \\
k_{c}^{-}=\frac{1}{2} k_{\mathrm{dec}, i n}
\end{gathered}
$$

for fast modes. Slow modes have the same

$$
k_{c}^{-}=2^{-\frac{3}{2}} k_{\mathrm{dec}, i n}
$$

as Alfvén modes, but the relation between $k_{c}^{+}$and $k_{\mathrm{dec}, n i}$ depends on the turbulence properties.

2. The single-fluid approach is capable of correctly describing wave behavior and damping properties in the strong coupling regime.

3. The cutoff of the MHD waves should also be taken into account when deriving the damping scales of turbulence cascades.

4. We showed the importance of neutral viscosity in damping Alfvén modes in the WNM and SC. In particular for the Alfvén modes in the SC, neutral viscosity is the dominant damping effect instead of ionneutral collisions.

5. We thoroughly investigated the behavior of slow waves and provided full expressions for the multiple cutoffs and wave frequencies in different coupling regimes.

6. The scale-dependent anisotropy of GS95-type turbulence is important for understanding wave behavior, calculating turbulence damping scales, and studying interactions between CRs and magnetic perturbations.

7. We evaluated the scattering efficiencies of TTD and gyroresonance with the three turbulence modes, and found that due to the severe damping in the partially ionized medium, only high-energy CRs can be effectively scattered through direct interactions with turbulence modes. Compared with other environments, CRs in the WNM are poorly confined due to its prominent turbulence anisotropy, as well as the largest damping scale of the fast modes. For CRs with lower energies, other effects such as field line wandering and streaming instability can set in and contribute to confining CRs.

S.X. and H.Y. are supported by NSFC grant AST-11473006. S.X. acknowledges support from the China Scholarship Council during her stay at the University of WisconsinMadison. S.X. also thanks Heshou Zhang for useful discussions. A.L. acknowledges NSF grant AST 1212096, a distinguished visitor PVE/CAPES appointment at the Physics Graduate Program of the Federal University of Rio Grande do Norte, and thanks the INCT INEspao and Physics Graduate Program/UFRN for hospitality.

\section{APPENDIX A}

\section{A SUMMARY OF THE NOTATION USED IN THIS PAPER}

\section{Drag coefficient}

Ion mass density

Neutral mass density

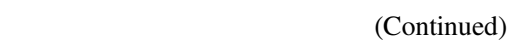

Total mass density

Proton mass

Mass of hydrogen atom

Ion mass

Neutral mass

Reduced mass

Ion number density

Neutral number density

Total number density

Ion-neutral collisional cross-section

Electronic charge

Neutral-ion collision frequency

Ion-neutral collision frequency

Ion fraction

Neutral fraction

Ratio of neutral and ion densities

Wave frequency

Real part of wave frequency

Imaginary part of wave frequency

Wavenumber

$x, y, z$ component of $k$

$k$ component parallel to local magnetic field

$k$ component perpendicular to local magnetic field

Wave propagation angle with respect to magnetic field

Alfvén speed

Alfvén speed of ion-electron gas

Alfvén speed of neutral gas

Magnetic field

Sound speed

Sound speed of neutral gas

Thermal speed of neutrals

Sound speed of ion-electron gas

Ion gas pressure

Neutral gas pressure

Temperature

Boltzmann constant

Adiabatic constant

Ratio of gas pressure to magnetic pressure

Injection scale of turbulence

Injection scale of MHD turbulence in super-Alfvénic turbulence

Transition scale from weak to strong MHD turbulence in sub-

Alfvénic turbulence

Turbulent velocity at $L$

Turbulent velocity at $l$

Turbulent velocity at $l_{\mathrm{tr}}$

Alfvénic Mach number

Damping scale

Ion velocity

Neutral velocity

Mean free path for a neutral particle

Cross-section for a neutral-neutral collision

Speed of light

Lorentz factor

CR particle's velocity

CR particle's gyrofrequency

CR particle's non-relativistic gyrofrequency

CR particle's pitch angle cosine

Pitch-angle diffusion coefficient

Scattering frequency

Growth rate of streaming instability

Streaming speed of CRs

Number density of CRs

Nonlinear damping rate 
APPENDIX B

APPROXIMATE SOLUTIONS FOR THE SINGLE-FLUID

\section{DISPERSION RELATION}

Here we list the analytical solutions to the single-fluid dispersion relation at some tractable limits following the method described in Section 3.2.

1. $\theta \rightarrow 0^{\circ}$

$$
\lambda_{1,2}= \pm c_{s n}, \lambda_{3,4}=\frac{V_{A n}}{2}\left(-i \tilde{k} \pm \sqrt{4-\tilde{k}^{2}}\right) .
$$

2. $\theta \rightarrow 90^{\circ}$

$$
\lambda_{1,2}= \pm c_{s n}
$$

3. $\beta \rightarrow 0$

$$
\begin{aligned}
& \lambda_{1,2}= \pm c_{s n} \cos \theta \\
& \lambda_{3,4}=\frac{V_{A n}}{2}\left(-i \tilde{k} \sec \theta \pm \sqrt{4-\tilde{k}^{2} \sec ^{2} \theta}\right) .
\end{aligned}
$$

4. $\beta \rightarrow \infty$

$\lambda_{1,2}= \pm c_{s n}, \lambda_{3,4}=-\frac{i V_{A n} \cos \theta\left(\tilde{k} \sec ^{2} \theta \pm \sqrt{\tilde{k}^{2} \sec ^{4} \theta-4}\right)}{2}$.

5. $\tilde{k} \rightarrow 0$ (ideal MHD case)

$$
\lambda_{1,2}= \pm \frac{\sqrt{c_{s n}^{2}+V_{A n}^{2}+\Delta}}{\sqrt{2}}, \lambda_{3,4}= \pm \frac{\sqrt{c_{s n}^{2}+V_{A n}^{2}-\Delta}}{\sqrt{2}} \text {. }
$$

where

$$
\Delta=\sqrt{\left(c_{s n}^{2}+V_{A n}^{2}\right)^{2}-4 c_{s n}^{2} V_{A n}^{2} \cos ^{2} \theta} .
$$

6. Small $\theta$ and $\beta \rightarrow 0$

$$
\begin{aligned}
\lambda_{1,2} & = \pm \frac{1+\cos ^{2} \theta}{2} c_{S n} \\
\lambda_{3} & =-\frac{V_{A n} \zeta}{2} i-\frac{4 \zeta-2 \tilde{k} \sec \theta \zeta^{2}+\zeta^{3}}{4\left(2 \zeta^{2}+3 \tilde{k} \sec \theta \zeta-4\right)} i \\
\lambda_{4} & =-\frac{V_{A n} \kappa}{2} i-\frac{4 \kappa-2 \tilde{k} \sec \theta \kappa^{2}+\kappa^{3}}{4\left(2 \kappa^{2}-3 \tilde{k} \sec \theta \kappa-4\right)} i
\end{aligned}
$$

where

$$
\zeta=\tilde{k}-i \sqrt{4-\tilde{k}^{2}}, \kappa=\tilde{k}+i \sqrt{4-\tilde{k}^{2}} .
$$

7. Small $\theta$ and $\beta \rightarrow \infty$

$$
\begin{aligned}
\lambda_{1,2} & = \pm c_{s n} \\
\lambda_{3} & =-\frac{V_{A n} \zeta}{2} i-\frac{4 \cos ^{2} \theta-2 \tilde{k} \sec \theta \zeta+\zeta^{2}}{4(\tilde{k} \sec \theta-\zeta)} i \\
\lambda_{4} & =-\frac{V_{A n} \kappa}{2} i-\frac{4 \cos ^{2} \theta-2 \tilde{k} \sec \theta \kappa+\kappa^{2}}{4(\tilde{k} \sec \theta-\kappa)} i
\end{aligned}
$$

8. $\theta \leqslant 90^{\circ}$

$$
\begin{aligned}
& \lambda_{1}=c_{S n}+\frac{c_{s n} \sin ^{2} \theta V_{A n}^{2}}{2\left(c_{s n}^{2}+i c_{s n} \tilde{k} \sec \theta V_{A n}-V_{A n}^{2}\right)}, \\
& \lambda_{2}=-c_{S n}-\frac{c_{s n} \sin ^{2} \theta V_{A n}^{2}}{2\left(c_{s n}^{2}-i c_{s n} \tilde{k} \sec \theta V_{A n}-V_{A n}^{2}\right)}, \\
& \lambda_{3}=-\frac{i V_{A n}}{\tilde{k} \sec ^{3} \theta} .
\end{aligned}
$$

9. Low $\beta$ and $\theta \rightarrow 0$

$$
\lambda_{1,2}= \pm c_{s n}, \lambda_{3}=-\frac{V_{A n} \zeta}{2} i, \lambda_{4}=-\frac{V_{A n} \kappa}{2} i .
$$

10. Low $\beta$ and $\theta \rightarrow 90^{\circ}$

$$
\lambda_{1,2}=\frac{V_{A n}}{2}\left(-i \tilde{k} \tan \theta \pm \sqrt{4-\tilde{k}^{2} \tan \theta^{2}}\right) .
$$

11. High $\beta$ and $\theta \rightarrow 0$

$$
\lambda_{1,2}= \pm c_{s n}, \lambda_{3,4}=-\frac{V_{A n}\left(\tilde{k} \pm \sqrt{\tilde{k}^{2}-4}\right)}{2} i .
$$

12. High $\beta$ and $\theta \rightarrow 90^{\circ}$

$$
\lambda_{1,2}= \pm c_{s n}, \lambda_{3}=-i V_{A n} \tilde{k} \sec \theta .
$$

13. Small $\tilde{k}$ and $\beta \rightarrow 0$

$$
\begin{aligned}
\lambda_{1,2} & = \pm \frac{c_{s n}}{\sqrt{2}}, \\
\lambda_{3} & =V_{A n}-\frac{i \tilde{k} V_{A n} \sec \theta}{3 i \tilde{k} \sec \theta+2}, \\
\lambda_{4} & =-V_{A n}+\frac{\tilde{k} V_{A n} \sec \theta}{3 \tilde{k} \sec \theta+2 i} .
\end{aligned}
$$

14. Small $\tilde{k}$ and $\beta \rightarrow \infty$

$$
\begin{aligned}
\lambda_{1,2} & = \pm c_{S n}, \\
\lambda_{3} & =\frac{V_{A n}}{\sqrt{2}}-\frac{i \tilde{k} V_{A n} \sec \theta}{\sqrt{2}(i \bar{k} \sec \theta+\sqrt{2})}, \\
\lambda_{4} & =-\frac{V_{A n}}{\sqrt{2}}-\frac{i \tilde{k} V_{A n} \sec \theta}{\sqrt{2}(-i \tilde{k} \sec \theta+\sqrt{2})} .
\end{aligned}
$$

15. Small $\tilde{k}$ and $\theta \rightarrow 0$

$$
\begin{aligned}
\lambda_{1,2} & = \pm c_{s n}, \\
\lambda_{3} & =V_{A n}+\frac{i \tilde{k} V_{A n}^{2}\left(V_{A n}^{2}-c_{s n}^{2}\right)}{i \tilde{k} V_{A n}\left(c_{s n}^{2}-3 V_{A n}^{2}\right)+2 V_{A n}\left(c_{s n}^{2}-V_{A n}^{2}\right)}, \\
\lambda_{4} & =-V_{A n}+\frac{i \tilde{k} V_{A n}^{2}\left(V_{A n}^{2}-c_{s n}^{2}\right)}{-i \tilde{k} V_{A n}\left(c_{s n}^{2}-3 V_{A n}^{2}\right)+2 V_{A n}\left(c_{s n}^{2}-V_{A n}^{2}\right)} .
\end{aligned}
$$

16. Small $\tilde{k}$ and $\theta \rightarrow 90^{\circ}$

$$
\lambda_{1,2}= \pm \frac{2\left(V_{A n}^{2}+c_{s n}^{2}\right)^{\frac{3}{2}}}{3 V_{A n}^{2}+2 c_{s n}^{2}} .
$$

\section{APPENDIX C}

DERIVATION OF THE GENERAL DISPERSION RELATION OF ALFVÉN WAVES CONSIDERING BOTH ION-NEUTRAL COLLISIONAL AND NEUTRALVISCOUS DAMPING

The momentum equations for ions and neutrals incorporating both ion-neutral friction and neutral-viscous terms are

$$
\begin{aligned}
& \rho_{i} \frac{\partial \boldsymbol{v}_{i}}{\partial t}+\rho_{i}\left(\boldsymbol{v}_{i} \cdot \nabla\right) \boldsymbol{v}_{i}=-\nabla P_{i}+\frac{1}{4 \pi}(\nabla \times \boldsymbol{B}) \\
& \times \boldsymbol{B}-\rho_{i} \nu_{\text {in }}\left(\boldsymbol{v}_{i}-\boldsymbol{v}_{n}\right), \\
& \rho_{n} \frac{\partial \boldsymbol{v}_{n}}{\partial t}+\rho_{n}\left(\boldsymbol{v}_{n} \cdot \nabla\right) \boldsymbol{v}_{n}=-\nabla P_{n}-\rho_{n} \nu_{n i}\left(\boldsymbol{v}_{n}-\boldsymbol{v}_{i}\right) \\
& -\rho_{n} \nu_{n} \nabla^{2} \boldsymbol{v}_{n},
\end{aligned}
$$


where $v_{i}, P i$, and $v_{n}, P_{n}$ are the velocity and pressure of the ions and neutrals, respectively. The induction equation is

$$
\frac{\partial \boldsymbol{B}}{\partial t}=\nabla \times\left(\boldsymbol{v}_{i} \times \boldsymbol{B}\right) .
$$

To perform a linear stability analysis, we consider linear perturbations superimposed on a static and homogeneous configuration,

$$
\begin{aligned}
& \boldsymbol{v}_{i}=\delta \boldsymbol{v}_{i}, \quad \boldsymbol{v}_{n}=\delta \boldsymbol{v}_{n}, \\
& \rho_{i}=\rho_{i 0}+\delta \rho_{i}, \quad \rho_{n}=\rho_{n 0}+\delta \rho_{n}, \boldsymbol{B}=\boldsymbol{B}_{0}+\delta \boldsymbol{B},
\end{aligned}
$$

where $\boldsymbol{B}_{0}, \rho_{i 0}$, and $\rho_{n 0}$ are the magnetic field, ion and neutral mass densities at the equilibrium state, and $\delta \boldsymbol{B}, \delta \rho_{i}$, and $\delta \rho_{n}$ are the corresponding perturbed variables. $\delta \boldsymbol{v}_{i}$ and $\delta \boldsymbol{v}_{n}$ are the ion and neutral velocity perturbations. We assume the background magnetic field is orientated along the $z$-direction and waves propagate in the $x-z$ plane (e.g., $k^{2}=k_{x}^{2}+k_{z}^{2}$ ). Thus the transverse Alfvén waves are polarized along the $y$-axis. By inserting Equation (105) into Equations (103) and (104), we deduce a set of linearized equations governing the $y$ components of perturbations,

$$
\begin{aligned}
\rho_{i 0} \frac{\partial \delta v_{i y}}{\partial t}= & \frac{B_{0}}{4 \pi} \frac{\partial \delta B_{y}}{\partial z}-\nu_{n i} \rho_{n 0}\left(\delta v_{i y}-\delta v_{n y}\right) \\
\rho_{n 0} \frac{\partial \delta v_{n y}}{\partial t}= & \nu_{n i} \rho_{n 0}\left(\delta v_{i y}-\delta v_{n y}\right) \\
& -\rho_{n 0} \nu_{n}\left(\frac{\partial^{2} \delta v_{n y}}{\partial x^{2}}+\frac{\partial^{2} \delta v_{n y}}{\partial y^{2}}+\frac{\partial^{2} \delta v_{n y}}{\partial z^{2}}\right), \\
& \frac{\partial \delta B_{y}}{\partial t}=B_{0} \frac{\partial \delta v_{i y}}{\partial z}
\end{aligned}
$$

Considering that the disturbance is proportional to $e^{i\left(k_{x} x+k_{z} z-\omega t\right)}$, the above equations yield the dispersion relation

$$
\begin{gathered}
\omega^{3}+i\left(\tau_{\nu}^{-1}+(1+\chi) \nu_{n i}\right) \omega^{2}-\left(k^{2} \cos ^{2} \theta V_{A i}^{2}+\chi \tau_{\nu}^{-1} \nu_{n i}\right) \omega \\
-i\left(\tau_{\nu}^{-1}+\nu_{n i}\right) k^{2} \cos ^{2} \theta V_{A i}^{2}=0 .
\end{gathered}
$$

When $\tau_{\nu}^{-1}=0$, the above dispersion relation recovers the classic one of Alfvén waves as shown in Equation (3).

\section{APPENDIX D \\ CRITICAL SCALES OF ION SLOW MODES CONSIDERING SCALE-DEPENDENT ANISOTROPY}

By substituting the scaling relations described in Equations (30) and (32) into Equations (62), (63), (67), and (68), we obtain expressions

$$
\begin{aligned}
& k_{c, t 1}=\left(\frac{\nu_{n i}}{2 \sqrt{\xi_{i}} c_{s i}}\right)^{\frac{3}{4}} L^{-\frac{1}{4}} M_{A}^{\frac{3}{4}}, \quad k_{c, t 2}=\left(\frac{2 \nu_{n i}}{\sqrt{\xi_{i}} c_{s i}}\right)^{\frac{3}{2}} L^{\frac{1}{2}} M_{A}^{-\frac{3}{2}}, \\
& k_{\mathrm{dec}, t 1}=\left(\frac{\nu_{n i}}{\sqrt{\xi_{i}} c_{s i}}\right)^{\frac{3}{4}} L^{-\frac{1}{4}} M_{A}^{\frac{3}{4}}, \quad k_{\mathrm{dec}, t 2}=\left(\frac{\nu_{n i}}{\sqrt{\xi_{i}} c_{s i}}\right)^{\frac{3}{2}} L^{\frac{1}{2}} M_{A}^{-\frac{3}{2}},
\end{aligned}
$$

for $1 / k<l_{A}$ in super-Alfvénic turbulence, and

$$
\begin{aligned}
& k_{c, t 1}=\left(\frac{\nu_{n i}}{2 \sqrt{\xi_{i}} c_{s i}}\right)^{\frac{3}{4}} L^{-\frac{1}{4}} M_{A}, \quad k_{c, t 2}=\left(\frac{2 \nu_{n i}}{\sqrt{\xi_{i}} c_{s i}}\right)^{\frac{3}{2}} L^{\frac{1}{2}} M_{A}^{-2}, \\
& k_{\mathrm{dec}, t 1}=\left(\frac{\nu_{n i}}{\sqrt{\xi_{i}} c_{s i}}\right)^{\frac{3}{4}} L^{-\frac{1}{4}} M_{A}, \quad k_{\mathrm{dec}, t 2}=\left(\frac{\nu_{n i}}{\sqrt{\xi_{i}} c_{s i}}\right)^{\frac{3}{2}} L^{\frac{1}{2}} M_{A}^{-2},
\end{aligned}
$$

for $1 / k<l_{\text {tr }}$ in sub-Alfvénic turbulence, under the consideration of strong turbulence anisotropy.

\section{APPENDIX E}

\section{FORCE ANALYSIS OF MAGNETOACOUSTIC WAVES}

The wave behavior can be better understood with the aid of force analysis. Here we follow the method in Soler et al. (2013b) for Alfvén waves to perform an analysis on the forces for magnetoacoustic waves. We start from the momentum equations of ions and neutrals (see, e.g., Zaqarashvili et al. 2011),

$$
\begin{gathered}
-i \omega v_{i x}=-i \frac{c_{s i}^{2} k_{x}^{2}}{\omega}\left(v_{i x}+v_{i z}\right)-i \frac{2 V_{A i}^{2} k_{x}^{2}}{\omega} v_{i x} \\
-\nu_{i n}\left(v_{i x}-v_{n x}\right), \\
-i \omega v_{i z}=-i \frac{c_{s i}^{2} k_{x}^{2}}{\omega}\left(v_{i x}+v_{i z}\right)-\nu_{i n}\left(v_{i z}-v_{n z}\right), \\
-i \omega v_{n x}=-i \frac{c_{s n}^{2} k_{x}^{2}}{\omega}\left(v_{n x}+v_{n z}\right)+\nu_{n i}\left(v_{i x}-v_{n x}\right), \\
-i \omega v_{n z}=-i \frac{c_{s n}^{2} k_{x}^{2}}{\omega}\left(v_{n x}+v_{n z}\right)+\nu_{n i}\left(v_{i z}-v_{n z}\right) .
\end{gathered}
$$

Here $v_{i}$ and $v_{n}$ are velocity perturbations in ions and neutrals. We consider that the constant magnetic field is along the $z$ direction, and waves propagate in $x-z$ plane. For a qualitative study of wave behavior, we fix the wave propagation direction as $45^{\circ}$ with respect to the magnetic field, i.e., $k_{x}=k_{z}$, to simplify the algebraic procedures. To conduct the force analysis for ion and neutral particles in different directions, from the above equations we obtain

$$
\begin{gathered}
f_{i, x}=\left|P_{i}\right|+|M|-\left|F_{x}\right|, \\
f_{i, z}=\left|P_{i}\right|-\left|F_{z}\right|, \\
f_{n, x}=\left|P_{n}\right|+\left|F_{x}\right|, \\
f_{n, z}=\left|P_{n}\right|+\left|F_{z}\right| .
\end{gathered}
$$

Here $\left|P_{i}\right|$ and $\left|P_{n}\right|$ are the gas pressure forces acting on ions and neutrals. $|M|$ is the magnetic pressure force and only acts on ions in the perpendicular direction to the magnetic field. $\left|F_{x}\right|$ and $\left|F_{z}\right|$ are friction forces between neutrals and ions in the $x$-and $z$ directions. Note that $\rho_{i}$ and $\rho_{n}$ omitted in Equation (112) are contained in the expressions of forces. After some algebraic manipulations, we next take the parameters of the MC phase and use the numerically solved $\omega$ of fast and slow waves to calculate the corresponding forces. To further simplify the problem, we also set $m_{i}=2 m_{n}$ to have equal sound speeds in the neutrals and ions. 
In Figure 16, we display the wave frequencies and different forces $|f|$ normalized by $|M|$ for magnetoacoustic waves using the parameters of the MC. The analytical expressions $\left|\omega_{R}\right|$ and $\left|\omega_{I}\right|$ of some branches are indicated.

(a) Fast waves. We see from Figure 16(b), $\left|F_{x}\right| \sim|M|$ (solid line) at large scales makes two fluids strongly coupled and oscillate together. The forces in Equation (113) satisfy

$$
\frac{f_{i, x}}{\rho_{i}} \approx \frac{f_{n, x}}{\rho_{n}}>\frac{f_{i, z}}{\rho_{i}} \approx \frac{f_{n, z}}{\rho_{n}}
$$

to make neutrals move together with ions. The perpendicular component of force is larger due to the involvement of magnetic pressure. $\left|F_{z}\right| /|M|$ (dashed line) increases toward $k_{\mathrm{dec}, n i}$ and exceeds $\left|P_{i}\right| /|M|$ (open circles) at $k_{\mathrm{dec}, n i}$. Within the cutoff interval, $\left|F_{x}\right| /|M|$ also increases to be larger than 1 . The strong friction force acting on ions efficiently dissipates the oscillatory motions of ions. In the meantime, due to the weak coupling with ions, $\left|P_{n}\right| /|M|$ (filled circles) in neutrals substantially drops to the same level as $\left|P_{i}\right| /|M|$ after the cutoff interval, and further decreases together with $\left|F_{z}\right| /|M|$ toward smaller scales. Accordingly, no wave motions can be developed in neutrals after they decouple from ions at $k_{\mathrm{dec}, n i}$.

At scales smaller than $1 / k_{\mathrm{dec}, \text { in }}$, the forces have relations

$$
\begin{gathered}
\frac{f_{i, x}}{\rho_{i}} \gg \frac{f_{n, x}}{\rho_{n}}, \frac{f_{i, z}}{\rho_{i}} \gg \frac{f_{n, z}}{\rho_{n}}, \\
f_{i, x} \gg f_{i, z}, f_{n, x} \gg f_{n, z} .
\end{gathered}
$$

With decreasing frictions in both directions, ions oscillate under the pressure forces and are marginally affected by neutrals. The damped fast waves re-emerge, but only in ions, with a phase speed $V_{A i}$ instead of $V_{A}$ (shown in Figure 16(a)).

(b) Usual slow waves. Owning to the constant propagation direction $\left(\theta=45^{\circ}\right)$ adopted, we observe different wave behaviors of the slow waves here (Figure 16(c)) from those in Figure 7 despite the same environment parameters used. First, since the relation $k_{\|} \ll k_{\perp} \sim k$ breaks down, $k_{\mathrm{dec}, n i}$ is smaller than $k_{c}^{+}$(see Equations (52) and (53)). Thus $k_{\mathrm{dec}, n i}$ becomes the cutoff scale of the usual slow waves in this case. Second, according to the expression in Table 4, the ratio $k_{c, t 1} / k_{c, t 2}$ substantially increases due to the relatively large value of $\cos \theta$. Meanwhile, the ratio $\left|\omega_{I}\right| /\left|\omega_{R}\right|$ of the ion slow waves within $\left[k_{c, t 1}, k_{c, t 2}\right]$ also increases, which leads to more efficient damping and a shorter wavenumber interval of this wave branch. In addition, with a constant $\cos \theta$, we cannot observe a decreasing $\left|\omega_{I}\right|$ with increasing $k$. From the above comparison we clearly see that the wave propagation direction can significantly influence the slow wave behavior.

Similar analysis to that of the fast modes applies to the usual slow modes on scales larger than $1 / k_{\mathrm{dec}, n i}$. Within the interval $\left[k_{\mathrm{dec}, n i}, k_{c, t 1}\right]$, ions are still strongly coupled with neutrals and undergo the same forces as neutrals, which we will discuss later for the neutral slow waves. The propagating ion slow waves arise at $k_{c, t 1}$ after $\left|P_{i}\right| /|M|$ substantially increases and $|M|$ dominates over $\left|F_{x}\right|$. But due to the increase of $\left|F_{z}\right| /|M|$, the wave is strongly damped with $\left|\omega_{I}\right|>\left|\omega_{R}\right|$ (see Figure 16(c)) and cut off quickly at $k_{c, t 2}$. The ion slow modes can only be fully resumed beyond $k_{\mathrm{dec}, \text { in }}$ when $\left|F_{z}\right| /|M|$ decreases to 1 . Then friction forces further decrease and become negligible compared with $|M|$ toward smaller scales. The forces have the relations

$$
\frac{f_{i, x}}{\rho_{i}}>\frac{f_{i, z}}{\rho_{i}} \gg \frac{f_{n, z}}{\rho_{n}}>\frac{f_{n, x}}{\rho_{n}} .
$$

(c) Neutral slow waves. As shown in Figure 16(e), with $\theta=45^{\circ}$, we can clearly see the change in the damping rate $\left|\omega_{I}\right|$ of the neutral slow modes (see Equations (58b) and (59)). Figure 16(f) illustrates the forces corresponding to the neutral slow modes. $\left|P_{n}\right| /|M|$ significantly increases and results in a nearly isotropic force on the neutrals. The compression produced by the usual slow modes at larger scales drives oscillations in neutrals. Without much interference from ions, isotropically propagating sound waves are able to arise and endure in the neutral fluid. Before reaching $k_{\mathrm{dec}, i n}$, ions are still coupled with neutrals. As a results, the forces are

$$
\frac{f_{n, x}}{\rho_{n}} \approx \frac{f_{n, z}}{\rho_{n}}>\frac{f_{i, x}}{\rho_{i}} \gg \frac{f_{i, z}}{\rho_{i}} .
$$

At scales smaller than $k_{\mathrm{dec}, i n}^{-1},\left|F_{x}\right| /|M|$ drops and converges with $\left|F_{z}\right| /|M|$. Accordingly $f_{i, x} / \rho_{i}$ further decreases and becomes significantly smaller compared with the forces on neutrals.

\section{APPENDIX F}

\section{THE FOKKER-PLANCK DIFFUSION COEFFICIENTS}

(a) Super-Alfvénic turbulence. In the case of super-Alfvénic turbulence, since the magnetic perturbations over the scales $\left[L, l_{A}\right]$ make an insignificant contribution to scattering for CRs with $r_{L}<l_{A}$, we perform the integration to calculate $D_{\mu \mu}$ from the injection scale of GS95 turbulence $l_{A}$ to the damping scale of turbulence for the sake of simplicity. This simplification in our calculation does not affect the results as long as the CR energy is within the energy range under consideration.

In Alfvénic turbulence, by applying NLT, we can obtain

$$
\begin{aligned}
D_{\mu \mu}^{G}(\mathrm{NLT})= & \frac{4 v \sqrt{\pi}\left(1-\mu^{2}\right)}{3 L R^{2}} \int_{1}^{k_{\mathrm{dam}} L} x x_{\perp}^{-10 / 3} d x \\
& \times \int_{0}^{1}\left(1+\frac{\mu V_{A}}{v}\right)^{2} \frac{d \eta}{\eta \Delta \mu} \frac{J_{1}^{2}(w)}{w^{2}} \\
& \times \exp \left[-\frac{x_{\|}}{x_{\perp}^{2 / 3}}-\frac{\left(\mu-\frac{1}{\eta x R}\right)^{2}}{\Delta \mu^{2}}\right],
\end{aligned}
$$

where " $G$ " refers to gyroresonance. Note that in the above equation and the similar following equations, the term $x_{\perp}$ is put outside the integral over $\eta$ for the clarity of presentation. $\eta$ contained in $x_{\perp}$ should be taken into account when one calculates the integration. Here the dimensionless quantities are: $R=v /(\Omega L)$ (CR rigidity), $x=k L$, and $\eta=\cos \theta$. $\Delta \mu=\Delta v_{\|} / v$ represents the dispersion in particle pitch angles due to the nonlinear effect, where $\Delta v_{\|} \sim \sqrt{M_{A}} v_{\perp}$ is related to the magnetic perturbation (Voelk 1975; Yan \& Lazarian 2008). $J_{n}(w)$ is the Bessel function with $w=k_{\perp} v_{\perp} / \Omega$. In QLT, with 


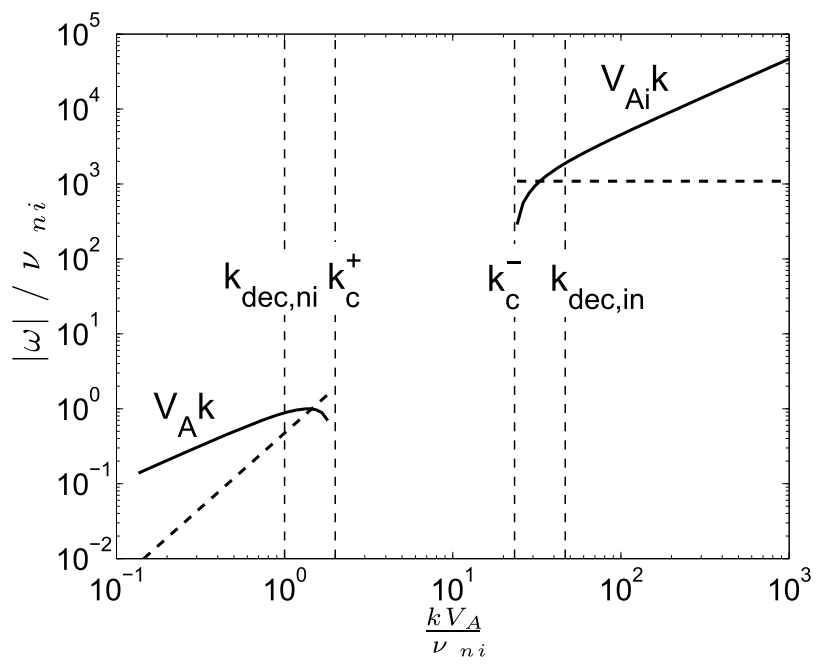

(a) Wave frequencies of fast modes

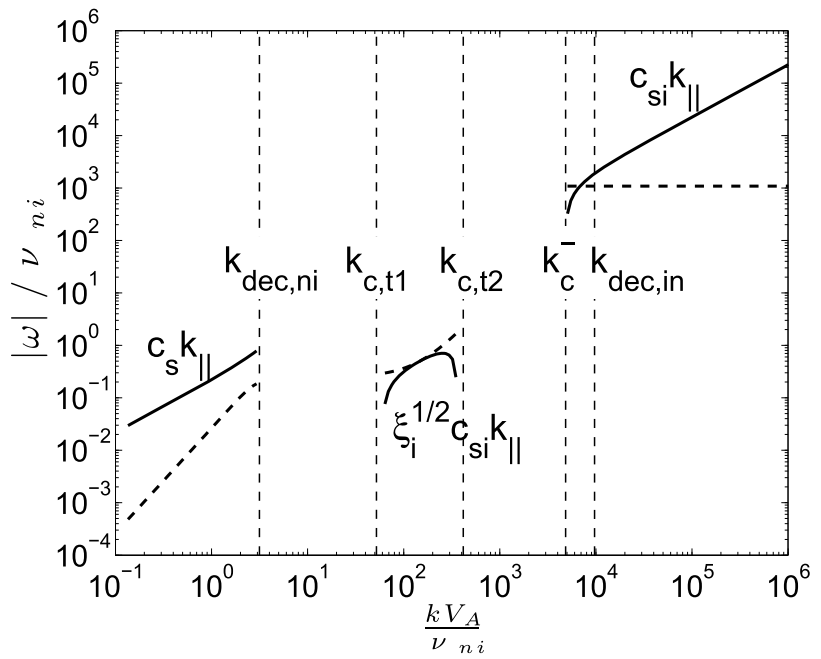

(c) Wave frequencies of usual slow modes

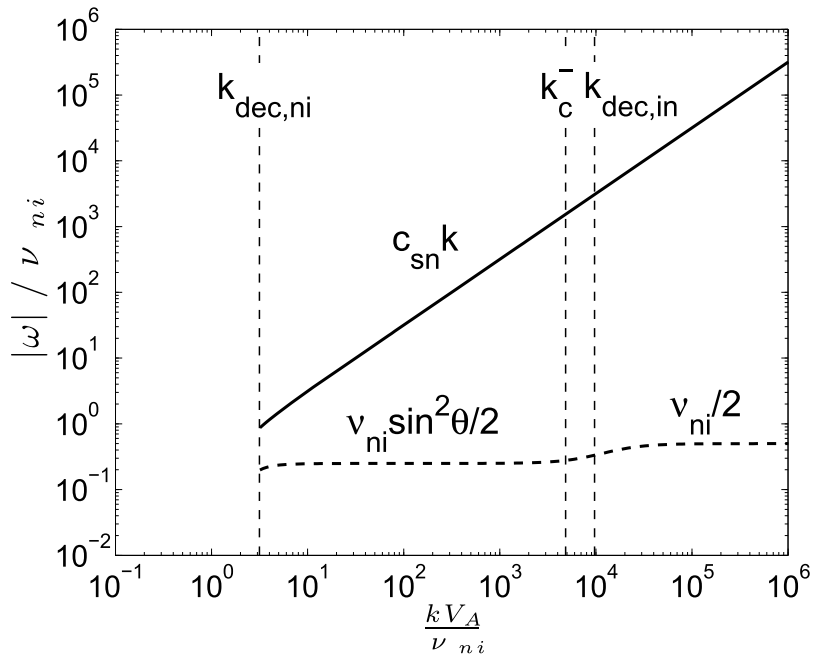

(e) Wave frequencies of neutral slow modes

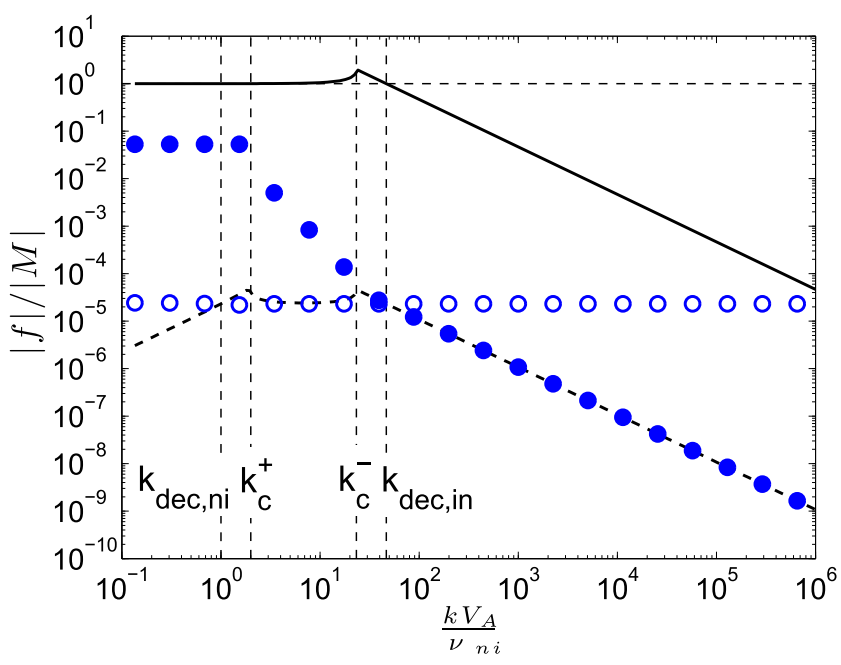

(b) Forces of fast modes

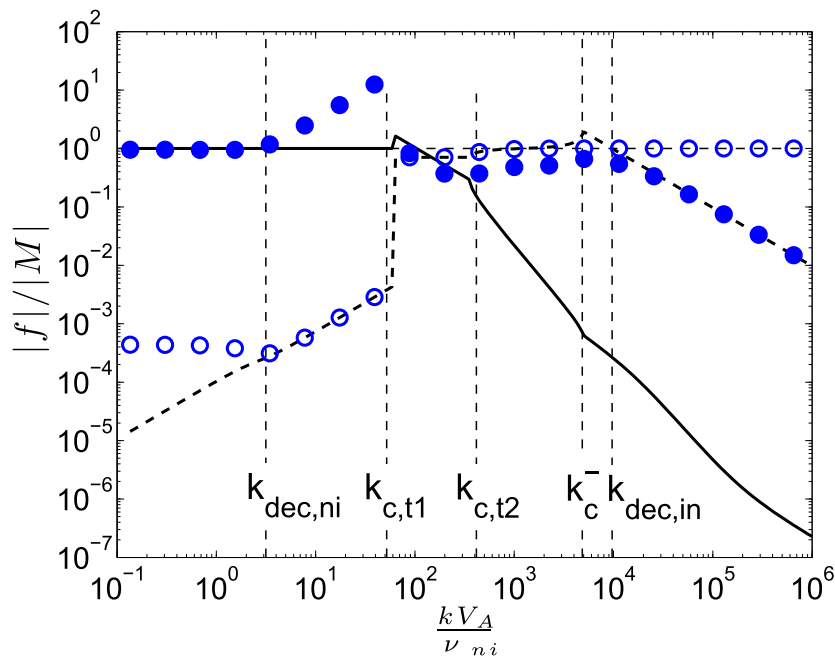

(d) Forces of usual slow modes

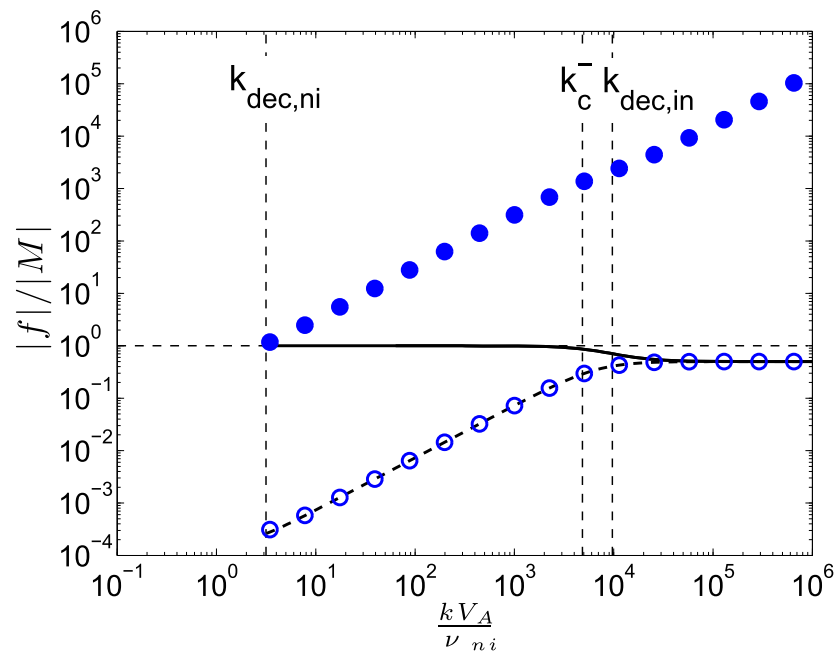

(f) Forces of neutral slow modes

Figure 16. Wave frequencies and force ratios vs. normalized $k$ of magnetoacoustic waves using MC parameters. In (a), (c), and (e), the propagating $\left(\left|\omega_{R}\right|\right)$ and damping $\left(\left|\omega_{I}\right|\right)$ components of wave frequencies are represented by the solid and dashed lines, respectively. In (b), (d), and (f), the displayed force ratios are $\left|F_{x}\right| /|M|($ solid line), $\left|F_{z}\right| /|M|$ (dashed line), $\left|P_{i}\right| /|M|$ (open circles), and $\left|P_{n}\right| /|M|$ (filled circles). The horizontal dashed lines indicate the position $|f| /|M|=1$. 
the $\delta$-function resonance condition used, $D_{\mu \mu}^{G}$ becomes

$$
\begin{aligned}
D_{\mu \mu}^{G}(\mathrm{QLT})= & \frac{4 v \pi\left(1-\mu^{2}\right)}{3 L R^{2}} \int_{1}^{k_{\mathrm{dam}} L} x^{2} x_{\perp}^{-10 / 3} d x \\
& \times \int_{0}^{1}\left(1+\frac{\mu V_{A}}{v}\right)^{2} \frac{d \eta}{\eta \mu} \frac{J_{1}^{2}(w)}{w^{2}} \\
& \times \exp \left(-\frac{x_{\|}}{x_{\perp}^{2 / 3}}\right) \delta\left(x-\frac{1}{\eta \mu R}\right) .
\end{aligned}
$$

For fast modes, we obtain

$$
\begin{aligned}
D_{\mu \mu}^{G}(\mathrm{NLT})= & \frac{v \sqrt{\pi}\left(1-\mu^{2}\right)}{L R^{2}} \int_{1}^{k_{\mathrm{dam}} L} x^{-5 / 2} d x \\
& \times \int_{0}^{1}\left(1+\frac{\mu V_{A}}{v \eta}\right)^{2} \frac{\eta d \eta}{\Delta \mu}\left(J_{1}{ }^{\prime}(w)\right)^{2} \\
& \left.\times \exp \left[-\frac{1}{\Delta \mu^{2}}\right)^{2}\right], \\
D_{\mu \mu}^{T}(\mathrm{NLT})= & \frac{v \sqrt{\pi}\left(1-\mu^{2}\right)}{L R^{2}} \int_{1}^{k_{\mathrm{dam}} L} x^{-5 / 2} d x \\
& \times \int_{0}^{1}\left(1+\frac{\mu V_{A}}{v \eta}\right)^{2} \frac{\eta d \eta}{\Delta \mu} J_{1}^{2}(w) \\
& \left.\times \exp \left[-\frac{V_{A}}{\eta \nu}\right)^{2}\right], \\
& {\left[\mu^{2}\right] }
\end{aligned}
$$

corresponding to gyroresonance and TTD interaction. For lowrigidity CRs with $r_{L}$ shorter than the damping scale of the turbulence, $J_{n}(w)$ can take the asymptotic form $(w / 2)^{n} / n$ ! at small argument. Then $D_{\mu \mu}^{T}$ (NLT) approximately becomes

$$
\begin{aligned}
D_{\mu \mu}^{T}(\mathrm{NLT})= & \frac{v \sqrt{\pi}\left(1-\mu^{2}\right)^{2}}{8 L \Delta \mu} \\
& \times \exp \left(-\frac{\mu^{2}}{(\Delta \mu)^{2}}\right)\left(\sqrt{k_{\mathrm{dam}} L}-1\right) .
\end{aligned}
$$

The $D_{\mu \mu}^{G}$ in QLT takes the form

$$
\begin{aligned}
& D_{\mu \mu}^{G}(\mathrm{QLT})=\frac{v \pi\left(1-\mu^{2}\right)}{L R^{2}} \int_{1}^{k_{\mathrm{dam}} L} x^{-3 / 2} d x \\
& \quad \times \int_{0}^{1}\left(1+\frac{\mu V_{A}}{v \eta}\right)^{2} \frac{\eta d \eta}{\mu}\left(J_{1}{ }^{\prime}(w)\right)^{2} \delta\left(x-\frac{1}{\eta \mu R}\right) .
\end{aligned}
$$

Its simplified form for low-rigidity CRs is

$$
D_{\mu \mu}^{G}(\mathrm{QLT})=\frac{v \pi \sqrt{\mu}\left(1-\mu^{2}\right)}{4 L \sqrt{R}}\left(\frac{2}{7}-\frac{2 \sqrt{1-\mu^{2}}}{21 \mu^{2}}\right) .
$$

The diffusion coefficients in the case of slow modes are

$$
\begin{aligned}
D_{\mu \mu}^{G}(\mathrm{NLT})= & \frac{v \sqrt{\pi}\left(1-\mu^{2}\right) \beta^{2}}{3 L R^{2}} \int_{1}^{k_{\mathrm{dam}} L} x x_{\perp}^{-10 / 3} d x \\
& \times \int_{0}^{1}\left(1+\frac{\mu c_{s}}{v}\right)^{2}\left(1-\eta^{2}\right) \frac{\eta^{3} d \eta}{\Delta \mu}\left(J_{1}^{\prime}(w)\right)^{2} \\
& \times \exp \left[-\frac{x_{\|}}{x_{\perp}^{2 / 3}}-\frac{\left(\mu-\frac{1}{\eta x R}\right)^{2}}{\Delta \mu^{2}}\right]
\end{aligned}
$$

$$
\begin{aligned}
D_{\mu \mu}^{T}(\mathrm{NLT})= & \frac{v \sqrt{\pi}\left(1-\mu^{2}\right) \beta^{2}}{3 L R^{2}} \int_{1}^{k_{\mathrm{dam}} L} x x_{\perp}^{-10 / 3} d x \\
& \times \int_{0}^{1}\left(1+\frac{\mu c_{s}}{v}\right)^{2}\left(1-\eta^{2}\right) \frac{\eta^{3} d \eta}{\Delta \mu} J_{1}^{2}(w) \\
& \times \exp \left[-\frac{x_{\|}}{x_{\perp}^{2 / 3}}-\frac{\left(\mu-\frac{c_{s}}{v}\right)^{2}}{\Delta \mu^{2}}\right] \\
D_{\mu \mu}^{G}(\mathrm{QLT})= & \frac{v \pi\left(1-\mu^{2}\right) \beta^{2}}{3 L R^{2}} \int_{1}^{k_{\mathrm{dam}} L} x^{2} x_{\perp}^{-10 / 3} d x \\
& \times \int_{0}^{1}\left(1+\frac{\mu c_{s}}{v}\right)^{2}\left(1-\eta^{2}\right) \frac{\eta^{3} d \eta}{\mu}\left(J_{1}{ }^{\prime}(w)\right)^{2} \\
& \times \exp \left(-\frac{x_{\|}}{x_{\perp}^{2 / 3}}\right) \delta\left(x-\frac{1}{\eta \mu R}\right)
\end{aligned}
$$

(b) Sub-Alfvénic turbulence. Sub-Alfvénic turbulence has different energy spectra for the weak and strong turbulence regimes. Accordingly, the diffusion coefficient is a sum of the components in both the weak and strong turbulence.

For the gyroresonance with Alfvén modes, we have $D_{\mu \mu}^{G}=D_{\mu \mu, w}^{G}+D_{\mu \mu, s}^{G}$. Here

$$
\begin{aligned}
D_{\mu \mu, w}^{G}(\mathrm{NLT})= & \frac{2 v \sqrt{\pi}\left(1-\mu^{2}\right) M_{A}^{2}}{L R^{2}} \\
& \times \int_{1}^{M_{A}^{-2}} x_{\perp}^{-2} d x_{\perp}\left(1+\frac{\mu V_{A}}{v}\right)^{2} \\
& \times \frac{1}{\Delta \mu} \frac{J_{1}^{2}(w)}{w^{2}} \exp \left[-\frac{\left(\mu-\frac{1}{R}\right)^{2}}{\Delta \mu^{2}}\right]
\end{aligned}
$$

is $D_{\mu \mu}^{G}$ in weak turbulence, and

$$
\begin{aligned}
D_{\mu \mu, s}^{G}(\mathrm{NLT})= & \frac{4 v \sqrt{\pi}\left(1-\mu^{2}\right) M_{A}^{4 / 3}}{3 L R^{2}} \int_{M_{A}^{-2}}^{k_{\mathrm{dam}} L} x x_{\perp}^{-10 / 3} d x \\
& \times \int_{0}^{1}\left(1+\frac{\mu V_{A}}{v}\right)^{2} \frac{d \eta}{\eta \Delta \mu} \frac{J_{1}^{2}(w)}{w^{2}} \\
& \times \exp \left[-\frac{x_{\|}}{x_{\perp}^{2 / 3} M_{A}^{4 / 3}}-\frac{\left(\mu-\frac{1}{\eta x R}\right)^{2}}{\Delta \mu^{2}}\right]
\end{aligned}
$$


is $D_{\mu \mu}^{G}$ in strong turbulence. We can see that $D_{\mu \mu, s}^{G}(\mathrm{NLT})$ is similar to $D_{\mu \mu}^{G}$ (NLT) in the super-Alfvénic case (Equation (118)), but with a factor $M_{A}^{4 / 3}$ added $\left(M_{A}<1\right)$.

In QLT, since weak turbulence only has smaller structures developed in the perpendicular direction, i.e., $k_{\|}=L^{-1}$ over $\left[L, l_{\mathrm{tr}}\right]$, weak turbulence does not contribute to gyroresonance scattering unless CRs have $r_{L}$ comparable to $L$. Thus we only consider the contribution of strong turbulence and obtain

$$
\begin{aligned}
D_{\mu \mu, s}^{G}(\mathrm{QLT})= & \frac{4 v \pi\left(1-\mu^{2}\right) M_{A}^{4 / 3}}{3 L R^{2}} \int_{M_{A}^{-2}}^{k_{\mathrm{dam}} L} x^{2} x_{\perp}^{-10 / 3} d x \\
& \times \int_{0}^{1}\left(1+\frac{\mu V_{A}}{v}\right)^{2} \frac{d \eta}{\eta \mu} \frac{J_{1}^{2}(w)}{w^{2}} \\
& \times \exp \left(-\frac{x_{\|}}{x_{\perp}^{2 / 3} M_{A}^{4 / 3}}\right) \delta\left(x-\frac{1}{\eta \mu R}\right) .
\end{aligned}
$$

The energy spectrum of fast modes is not dependent on turbulence regimes. Fast modes have similar diffusion coefficients in sub-Alfvénic turbulence as those in the superAlfvénic case. The expressions of Equations (120)-(74) still apply, but here $L$ is the driving scale of turbulence and $M_{A}^{2}$ should be multiplied accounting for the ratio between the kinetic and magnetic energies of turbulence.

Slow modes have

$$
\begin{aligned}
D_{\mu \mu, w}^{G}(\mathrm{NLT})= & \frac{v \sqrt{\pi}\left(1-\mu^{2}\right) \beta^{2} M_{A}^{2}}{2 L R^{2}} \\
& \times \int_{1}^{M_{A}^{-2}} x_{\perp}^{-2} d x_{\perp}\left(1+\frac{\mu c_{s}}{v}\right)^{2}\left(1-\eta^{2}\right) \\
& \times \frac{\eta^{4}}{\Delta \mu}\left(J_{1}^{\prime}(w)\right)^{2} \exp \left[-\frac{\left(\mu-\frac{1}{R}\right)^{2}}{\Delta \mu^{2}}\right], \\
D_{\mu \mu, w}^{T}(\mathrm{NLT})= & \frac{v \sqrt{\pi}\left(1-\mu^{2}\right) \beta^{2} M_{A}^{2}}{2 L R^{2}} \\
& \times \int_{1}^{M_{A}^{-2}} x_{\perp}^{-2} d x_{\perp}\left(1+\frac{\mu c_{s}}{v}\right)^{2}\left(1-\eta^{2}\right) \\
& \times \frac{\eta^{4}}{\Delta \mu} J_{1}^{2}(w) \exp \left[-\frac{\left(\mu-\frac{c_{s}}{v}\right)^{2}}{\Delta \mu^{2}}\right]
\end{aligned}
$$

in weak turbulence, and

$$
\begin{aligned}
D_{\mu \mu, s}^{G}(\mathrm{NLT})= & \frac{v \sqrt{\pi}\left(1-\mu^{2}\right) \beta^{2} M_{A}^{4 / 3}}{3 L R^{2}} \int_{M_{A}^{-2}}^{k_{\mathrm{dam}} L} x x_{\perp}^{-10 / 3} d x \\
& \times \int_{0}^{1}\left(1+\frac{\mu c_{s}}{v}\right)^{2}\left(1-\eta^{2}\right) \frac{\eta^{3} d \eta}{\Delta \mu}\left(J_{1}{ }^{\prime}(w)\right)^{2} \\
& \times \exp \left[-\frac{x_{\|}}{x_{\perp}^{2 / 3} M_{A}^{4 / 3}}-\frac{\left(\mu-\frac{1}{\eta \times R}\right)^{2}}{\Delta \mu^{2}}\right]
\end{aligned}
$$

$$
\begin{aligned}
D_{\mu \mu, s}^{T}(\mathrm{NLT})= & \frac{v \sqrt{\pi}\left(1-\mu^{2}\right) \beta^{2} M_{A}^{4 / 3}}{3 L R^{2}} \int_{M_{A}^{-2}}^{k_{\mathrm{dam}} L} x x_{\perp}^{-10 / 3} d x \\
& \times \int_{0}^{1}\left(1+\frac{\mu c_{s}}{v}\right)^{2}\left(1-\eta^{2}\right) \frac{\eta^{3} d \eta}{\Delta \mu} J_{1}^{2}(w) \\
& \times \exp \left[-\frac{x_{\|}}{x_{\perp}^{2 / 3} M_{A}^{4 / 3}}-\frac{\left(\mu-\frac{c_{s}}{v}\right)^{2}}{\Delta \mu^{2}}\right]
\end{aligned}
$$

in strong turbulence.

QLT in strong turbulence gives

$$
\begin{aligned}
D_{\mu \mu}^{G}(\mathrm{QLT})= & \frac{v \pi\left(1-\mu^{2}\right) \beta^{2} M_{A}^{4 / 3}}{3 L R^{2}} \int_{M_{A}^{-2}}^{k_{\mathrm{dam}} L} x^{2} x_{\perp}^{-10 / 3} d x \\
& \times \int_{0}^{1}\left(1+\frac{\mu c_{s}}{v}\right)^{2}\left(1-\eta^{2}\right) \frac{\eta^{3} d \eta}{\mu}\left(J_{1}{ }^{\prime}(w)\right)^{2} \\
& \times \exp \left(-\frac{x_{\|}}{x_{\perp}^{2 / 3} M_{A}^{4 / 3}}\right) \delta\left(x-\frac{1}{\eta \mu R}\right) .
\end{aligned}
$$

\section{REFERENCES}

Ackermann, M., Ajello, M., Atwood, W. B., et al. 2012, ApJ, 750, 3 Adriani, O., Barbarino, G. C., Bazilevskaya, G. A., et al. 2011, Sci, 332, 69 Armstrong, J. W., Rickett, B. J., \& Spangler, S. R. 1995, ApJ, 443, 209 Balsara, D. S. 1996, ApJ, 465, 775

Beresnyak, A. 2014, ApJL, 784, L20

Beresnyak, A. 2015, ApJL, 801, L9

Beresnyak, A., \& Lazarian, A. 2008, ApJ, 682, 1070

Beresnyak, A., \& Lazarian, A. 2015, in Astrophysics and Space Science Library, Vol. 407, Magnetic Fields in Diffuse Media, ed. A. Lazarian,

E. M. de Gouveia Dal Pino, \& C. Melioli (Berlin: Springer), 163

Braginskii, S. I. 1965, RvPP, 1, 205

Brandenburg, A., \& Lazarian, A. 2014, in Astrophysical Hydromagnetic Turbulence, ed. A. Balogh et al. (New York: Springer), 87

Brunetti, G., \& Lazarian, A. 2007, MNRAS, 378, 245

Brunetti, G., \& Lazarian, A. 2011, MNRAS, 412, 817

Brunetti, G., \& Lazarian, A. 2016, MNRAS, 458, 2584

Bykov, A. M., \& Toptygin, I. N. 2001, AstL, 27, 625

Cesarsky, C. J. 1980, ARA\&A, 18, 289

Chandran, B. D. G. 2000, PhRvL, 85, 4656

Chepurnov, A., \& Lazarian, A. 2010, ApJ, 710, 853

Cho, J., \& Lazarian, A. 2002, PhRvL, 88, 245001

Cho, J., \& Lazarian, A. 2003, MNRAS, 345, 325

Cho, J., Lazarian, A., \& Vishniac, E. T. 2002, ApJ, 564, 291

Cho, J., Lazarian, A., \& Vishniac, E. T. 2003, in Turbulence and Magnetic Fields in Astrophysics, Vol. 614, ed. E. Falgarone, \& T. Passot (Berlin: Springer)

Cho, J., \& Vishniac, E. T. 2000, ApJ, 539, 273

Crutcher, R. M., Wandelt, B., Heiles, C., Falgarone, E., \& Troland, T. H. 2010, ApJ, 725, 466

De Pontieu, B., Carlsson, M., Rouppe van der Voort, L. H. M., et al. 2012, ApJL, 752, L12

De Pontieu, B., McIntosh, S. W., Carlsson, M., et al. 2007, Sci, 318, 1574

Draine, B. T. 2011, Physics of the Interstellar and Intergalactic Medium (Princeton, NJ: Princeton Univ. Press)

Draine, B. T., Roberge, W. G., \& Dalgarno, A. 1983, ApJ, 264, 485

Farmer, A. J., \& Goldreich, P. 2004, ApJ, 604, 671

Ferriere, K. M., Zweibel, E. G., \& Shull, J. M. 1988, ApJ, 332, 984

Forteza, P., Oliver, R., Ballester, J. L., \& Khodachenko, M. L. 2007, A\&A, 461, 731

Goldreich, P., \& Sridhar, S. 1995, ApJ, 438, 763

Goodman, M. L. 2000, ApJ, 533, 501

Goodman, M. L. 2001, SSRv, 95, 79

Guillian, G., Hosaka, J., Ishihara, K., et al. 2007, PhRvD, 75, 062003 
Hollweg, J. V. 1986, JGR, 91, 4111

Hunter, S. D., Bertsch, D. L., Catelli, J. R., et al. 1997, ApJ, 481, 205

Jokipii, J. R. 1966, ApJ, 146, 480

Khodachenko, M. L., Arber, T. D., Rucker, H. O., \& Hanslmeier, A. 2004, A\&A, 422, 1073

Kowal, G., \& Lazarian, A. 2010, ApJ, 720, 742

Kowal, G., Lazarian, A., Vishniac, E. T., \& Otmianowska-Mazur, K. 2009, RMxAA, 36, 89

Kowal, G., Lazarian, A., Vishniac, E. T., \& Otmianowska-Mazur, K. 2012, NPGeo, 19, 297

Kulsrud, R., \& Pearce, W. P. 1969, ApJ, 156, 445

Kulsrud, R. M., \& Anderson, S. W. 1992, ApJ, 396, 606

Kumar, N., \& Roberts, B. 2003, SoPh, 214, 241

Langer, W. D. 1978, ApJ, 225, 95

Lazarian, A. 2006, ApJL, 645, L25

Lazarian, A., \& Beresnyak, A. 2006, MNRAS, 373, 1195

Lazarian, A., Esquivel, A., \& Crutcher, R. 2012, ApJ, 757, 154

Lazarian, A., \& Vishniac, E. T. 1999, ApJ, 517, 700

Lazarian, A., Vishniac, E. T., \& Cho, J. 2004, ApJ, 603, 180

Lazarian, A., \& Yan, H. 2014, ApJ, 784, 38

Leake, J. E., \& Arber, T. D. 2006, A\&A, 450, 805

Li, P. S., McKee, C. F., Klein, R. I., \& Fisher, R. T. 2008, ApJ, 684, 380

Lithwick, Y., \& Goldreich, P. 2001, ApJ, 562, 279

L'vov, V. S., L'vov, Y. V., \& Pomyalov, A. 2000, PhRvE, 61, 2586

Maron, J., \& Goldreich, P. 2001, ApJ, 554, 1175

Mouschovias, T. C., Ciolek, G. E., \& Morton, S. A. 2011, MNRAS, 415, 1751

Narain, U., \& Ulmschneider, P. 1996, SSRv, 75, 453

Narayan, R., \& Medvedev, M. V. 2001, ApJL, 562, L129

Okamoto, T. J., \& De Pontieu, B. 2011, ApJL, 736, L24

Oishi, J. S., \& Mac Low, M.-M. 2006, ApJ, 638, 281

Osterbrock, D. E. 1961, ApJ, 134, 347

Piddington, J. H. 1956, MNRAS, 116, 314

Pudritz, R. E. 1990, ApJ, 350, 195
Schlickeiser, R. 2002, Cosmic Ray Astrophysics, ed. R. Schlickeiser (Berlin: Springer)

Schlickeiser, R., \& Miller, J. A. 1998, ApJ, 492, 352

Shu, F. H. 1992, in The Physics of Astrophysics, Volume II: Gas Dynamics (Mill Valley, CA: Univ. Science Books), 360

Soler, R., Ballester, J. L., \& Zaqarashvili, T. V. 2015, A\&A, 573, A79

Soler, R., Carbonell, M., \& Ballester, J. L. 2013a, ApJS, 209, 16

Soler, R., Carbonell, M., Ballester, J. L., \& Terradas, J. 2013b, ApJ, 767, 171

Spitzer, L. 1978, Physical Processes in the Interstellar Medium (New York: Wiley)

Tilley, D. A., \& Balsara, D. S. 2008, MNRAS, 389, 1058

Vernazza, J. E., Avrett, E. H., \& Loeser, R. 1981, ApJS, 45, 635

Voelk, H. J. 1975, RvGSP, 13, 547

Vranjes, J. 2014, MNRAS, 445, 1614

Vranjes, J., \& Krstic, P. S. 2013, A\&A, 554, A22

Wentzel, D. G. 1974, ARA\&A, 12, 71

Xu, S., Lazarian, A., \& Yan, H. 2014, arXiv:1412.8608

Yan, H., \& Lazarian, A. 2002, PhRvL, 89, B1102

Yan, H., \& Lazarian, A. 2003, ApJL, 592, L33

Yan, H., \& Lazarian, A. 2004, ApJ, 614, 757

Yan, H., \& Lazarian, A. 2008, ApJ, 673, 942

Yan, H., \& Lazarian, A. 2011, ApJ, 731, 35

Yan, H., \& Lazarian, A. 2012, in ASP Conf. Ser. 459, Numerical Modeling of Space Plasma Slows (ASTRONUM 2011), ed. N. V. Pogorelov et al. (San Francisco, CA: ASP), 40

Yan, H., Lazarian, A., \& Petrosian, V. 2008, ApJ, 684, 1461

Yan, H., Lazarian, A., \& Schlickeiser, R. 2012, ApJ, 745, 140

Zakharov, V. E. 1967, JETP, 24, 455

Zakharov, V. E., \& Sagdeev, R. Z. 1970, SPhD, 15, 439

Zaqarashvili, T. V., Carbonell, M., Ballester, J. L., \& Khodachenko, M. L. 2012, A\&A, 544, A143

Zaqarashvili, T. V., Khodachenko, M. L., \& Rucker, H. O. 2011, A\&A, 529, A82 